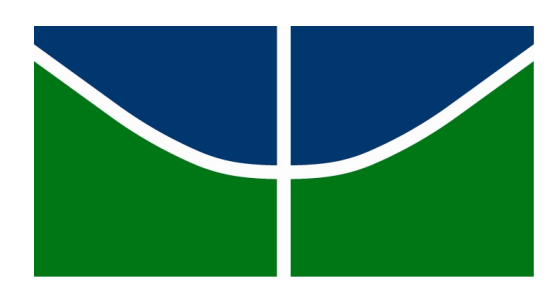

\author{
Universidade de Brasília \\ Instituto de Ciências Exatas \\ Departamento de Matemática
}

\title{
Solitons de Yamabe e Métricas CPE
}

por

Benedito Leandro Neto

Brasília 
Universidade de Brasília

Instituto de Ciências Exatas

Departamento de Matemática

\title{
Solitons de Yamabe e Métricas CPE
}

\author{
por
}

\section{Benedito Leandro Neto}

Tese apresentada ao Departamento de Matemática da Universidade de Brasília como parte dos requisitos necessários para obtenção do grau de

\section{DOUTOR EM MATEMÁTICA}

24 de novembro de 2015

Comissão Examinadora:

\author{
$\overline{\text { Profa. Dra. Keti Tenenblat - Orientadora (MAT-UnB) }}$ \\ $\overline{\text { Prof. Dr. Ernani Ribeiro Júnior (MAT-UFC) }}$
}

Prof. Dr. Romildo da Silva Pina (IME-UFG)

Prof. Dr. Detang Zhou (CEG-UFF)

$\overline{\text { Prof. Dr. Xia Chang Yu (MAT-UnB) }}$

*O autor foi bolsista CAPES e CNPq durante a elaboração deste trabalho. 
À minha mãe,

Teresinha Aparecida Correa Leandro. 
"Se as portas da percepção estivessem limpas, tudo se mostraria ao homem tal como é: infinito". (William Blake) 


\section{Agradecimentos}

Eu agradeço a minha mãe, Teresinha Aparecida Correa Leandro, em primeiro lugar. Ela não me deixou desistir dessa empreitada, mesmo na hora mais escura de nossas vidas. Eu tenho certeza que essa tese é muito mais uma obra dela do que minha. Sinto sua falta todos os dias.

Aos meus afilhados, Nicolau, Vinícius e Eduardo. Que me deram algo primordial, alegria. Um sorriso dessa "garotada"recarrega minhas energias.

Quero agradecer a Carolina M. Carvalho e toda sua família pelo apoio e carinho.

Eu agradeço ao meu pai e aos meus irmãos, por terem me apoiado.

Não posso deixar de agradecer ao meu orientador de mestrado Romildo da Silva Pina. E minha orientadora de doutorado, Keti Tenenblat. Eles me deram uma oportunidade única e acreditaram em mim. A vocês dois, muito obrigado.

Ao meu colega, Professor Ernani Ribeiro junior. Muito obrigado por ter paciência comigo e por também ter me guiado nessa caminhada.

Aos meus grandes amigos que me acompanharam nesses quatro anos Gabriela Ferreira Nogueira, Bruno Trindade, Agenor, Alex Teló, Alex Paraiba, Newton Mayer, Tarcísio, José Carlos, Emerson, Caike, Isabela Rezende e Pablo Italiano, obrigado pelas discussões e companheirismo.

Aos meus amigos de infância Maíra Branquinho Cardoso, Marcos Vinícius Alves da Costa, Rafael Gargano, Thiago Rocha, Tereza Cristina, Joana Tábata um abraço e meu carinho sempre.

Agradeço ao CNPq/CAPES pelo suporte financeiro durante um período do doutoramento.

A todos vocês, muito obrigado. 


\section{Resumo}

Provamos que (anti)self dual solitons gradientes (quasi) Yamabe com curvatura seccional positiva são rotacionais simétricos. Além disso, mostramos que, (anti)self dual solitons gradientes de Yamabe tem uma estrutura de produto torcido muito particular desde que a função potencial não tenha pontos críticos.

Consideramos solitons gradientes de Yamabe conformes a espaços pseudo-Euclidianos $n$-dimensionais. Caracterizamos todos os solitons que são invariantes pela ação de um grupo de translação $(n-1)$-dimensional e obtemos os solitons tipo estável. Como aplicação, obtemos um exemplo de soliton gradiente de Yamabe estável completo, conforme a um espaço Lorentziano.

Investigamos também os pontos críticos do funcional curvatura escalar total restrito a um espaço de métricas com curvatura escalar constante e volume unitátio que denotamos por métricas CPE. Foi conjecturado nos anos 1980 que toda métrica CPE é, necessariamente, Einstein. Provamos que métricas CPE, no caso de dimensão quatro, com tensor $W^{+}$harmônico são isométricas à esfera $\mathbb{S}^{4}$ dada uma condição integral. No caso $n$-dimensional, provamos uma condição necessária e suficiente sobre a norma do gradiente da função potencial para que a métrica CPE seja Einstein.

Palavras-chave: Solitons de Yamabe; métricas Lorentzianas; mética conforme; funcional curvatura escalar total; Métrica CPE; Métricas de Einstein; soliton quasi Yamabe; localmente conformemente plano; self dual. 


\section{Abstract}

We prove that an (anti)self dual quasi Yamabe gradient soliton with positive sectional curvature is rotationally symmetric. We also prove that four dimensional (anti) self dual gradient Yamabe soliton has a special warped product structure provided that the potential function has no critical points.

We consider gradient Yamabe solitons, conformal to an $n$-dimensional pseudoEuclidean space. We characterize all such solitons which are invariant under the action of an $(n-1)$-dimensional translation group and we obtain the steady solitons. Applications provide an explicit example of a complete steady gradient Yamabe soliton, conformal to the Lorentzian space.

We also investigate the critical points of the total scalar curvature functional restricted to space of metrics with constant scalar curvature of unitary volume, for simplicity CPE metrics. It was conjectured in the 1980's that every CPE metric must be Einstein. We prove that a 4-dimensional CPE metric with harmonic tensor $W^{+}$must be isometric to a round sphere $\mathbb{S}^{4}$ provided an integral condition is satisfied. We also give a necessary and sufficient condition on the norm of the gradient of the potential function for a CPE metric to be Einstein.

Keywords: Yamabe solitons; Lorentzian metrics; conformal metrics; total scalar curvature functional; critical point equation; Einstein metric; quasi Yamabe soliton; half conformally flat; self dual. 


\section{Sumário}

1 Preliminares 14

1.1 Variedades semi-Riemannianas, métricas conformes e Tensores . . . . . . 14

1.2 Teorema de Stokes . . . . . . . . . . . . . . . . 20

1.3 Teoria dos grupos de simetria . . . . . . . . . . . . . 22

1.4 Soliton gradiente quasi Yamabe .................. 25

1.5 Estrutura do produto torcido . . . . . . . . . . . 27

1.6 Variedades de dimensão 4 e suas propriedades especiais . . . . . . . . . 29

1.7 Métrica CPE (critical point equation) . . . . . . . . . . . 32

2 Solitons gradientes de Yamabe conformes a um espaço pseudo-Euclidiano 34

2.1 Demonstração dos resultados principais . . . . . . . . . . . . . 39

2.2 Exemplo de soliton gradiente de Yamabe semi-Riemanniano estável com-

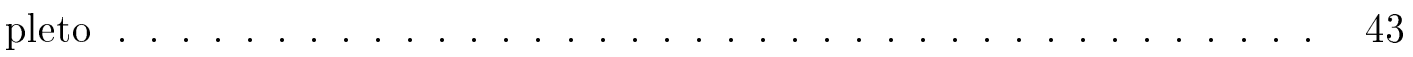

3 Soliton gradiente quasi Yamabe em dimensão $n=4 \quad 49$

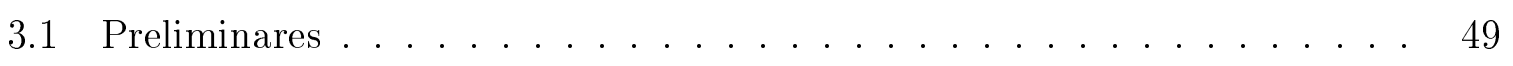

3.2 Resultados principais . . . . . . . . . . . . . . . 58

4 Estudo da função potencial da métrica CPE 60

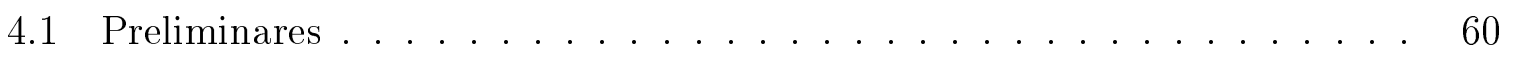

4.2 Resultados principais . . . . . . . . . . . . . . . 62 
5 Métrica CPE em dimensão $n=4$

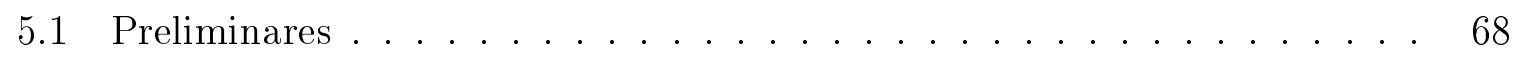

5.2 Resultados principais . . . . . . . . . . . . . . . 70

Referências Bibliográficas 


\section{Introdução}

O fluxo de Yamabe e o fluxo de Ricci, foram introduzidos como uma tentativa de se resolver o problema de Yamabe e a conjectura de Poincaré, respectivamente (ver [43]). Nesse contexto, os solitons de Ricci, soluções auto-similares para o fluxo de Ricci ([29]), são ferramentas importantes para a matemática contemporânea. A maior parte do trabalho foi feito no caso Riemanniano (veja por exemplo [16] e [13]). Entretanto, solitons de Ricci foram considerados, recentemente, no caso Lorentziano ([3, 10, 27, 49]).

Motivado pela classificação dos solitons de Ricci localmente conformemente planos, Daskalopoulos e Sesum [25] iniciaram uma investigação dos solitons de Yamabe conformemente planos e demonstraram que todo soliton gradiente de Yamabe completo, localmente conformemente plano com curvatura seccional positiva é rotacionalmente simétrico. Além disso, eles construíram exemplos de solitons de Yamabe rotacionalmente simétricos em $\mathbb{R}^{n}$ com curvatura seccional positiva. Inspirado por esses autores, Cao, Sun e Zhang [15] mostraram que todo soliton gradiente de Yamabe não-trivial admite uma estrutura global especial de produto torcido. Vale lembrar que os solitons gradientes de Yamabe compactos possuem curvatura escalar constante (veja por exemplo [24]).

Nesse trabalho, vamos estudar solitons de Yamabe e métricas CPE (critical point equation [9] e [29]).

Um dos nossos objetivos é caracterizar solitons de Yamabe gradiente conformes a espaços pseudo-Euclidianos $n$-dimensionais, que são invariantes pela ação de um grupo de translação $(n-1)$-dimensional (veja os Teoremas 2.3 e 2.4). Barbosa, Pina e Tenenblat [3] consideraram esse problema para os solitons de Ricci. Precisamente, eles estudaram solitons gradientes de Ricci, conformes a espaços pseudo- Euclidianos invariantes pela ação de um grupo de translação $(n-1)$-dimensional, encontrando todas as soluções no caso do soliton gradiente de Ricci estável.

Quando $M$ é uma variedade Riemanniana a definição exige que a variedade seja completa. No caso semi-Riemanniano, não exigimos que $(M, g)$ seja completa [12]. Em geral, para uma variedade indefinida, o termo "completo" significa apenas geodesicamente completo, já que não existe uma "distância" canônica associada à métrica. Por outro 
lado, existem geodésicas tipo tempo, espaço e luz. Logo, podemos falar em completude tipo tempo, espaço ou luz, dependendo de qual tipo de geodésica estamos considerando. Esperava-se que um certo tipo de completude (geodesicamente completo) implicasse em outro tipo de completude (veja [7]). Mas, existem exemplos explícitos que mostram a independência lógica dos três tipos de completude.

Completude geodésica em variedade semi-Riemanniana é um conceito importante quando estamos interessados em definir uma singularidade em relatividade geral. Além disso, obter explicitamente as geodésicas e suas singularidades, num espaço semi-Riemanniano é uma tarefa difícil.

No Capítulo 2, vamos considerar solitons gradientes de Yamabe, conformes a um espaço pseudo-Euclidiano. Caracterizamos todas as soluções invariantes pela ação do grupo de translação $(n-1)$-dimensional, Teoremas 2.1 e 2.2, e vamos obter todos solitons de Yamabe estáveis, Teoremas 2.3 e 2.4. Como uma aplicação do Teorema 2.4, na Seção 2.2 do Capítulo 2, nós obtemos um exemplo de soliton gradiente de Yamabe estável completo, conforme a um espaço Lorentziano $\mathbb{L}^{n}$. Mostramos que esse soliton gradiente de Yamabe é geodesicamente completo, que sua curvatura seccional assume qualquer valor real, encontraremos seu tensor de Ricci e, com isso, veremos que sua curvatura escalar é constante.

As métricas do tipo soliton gradiente de Yamabe podem ser estendidas naturalmente (ver [17]). Essa extensão é uma idéia que apareceu no estudo das variedades com peso (ver [58]), onde as métricas quasi Einstein surgem como generalização das métricas de Einstein. Com base nesses conceitos, definiu-se as métricas do tipo soliton gradiente quasi Yamabe (ver [30] e [57]). Essas métricas tipo Einstein tem sido estudadas hoje em dia, e vários resultados de classificação já foram obtidos. Na verdade, o estudo dessas métricas tipo Einstein é um problema antigo (veja cap.16 [9]).

No Capítulo 3, obtemos uma classificação dos solitons gradientes quasi Yamabe para o caso de dimensão quatro. Mostramos que a condição localmente conformemente plana pode ser substituída pela condição mais fraca semi conformemente plana (ou self dual tensor de Weyl). Vale lembrar que $\mathbb{C P} 2$ é um exemplo de variedade que é localmente semi conformemente plana mas não é localmente conformemente plana. No Teorema 3.6 e no Corolário 3.7 mostramos que, com a hipótese semi conformemente plano, o soliton gradiente (quasi) Yamabe é rotacionalmente simétrico quando sua curvatura seccional é positiva. No Teorema 3.8 mostramos que (anti)self dual solitons gradientes de Yamabe tem uma estrutura de produto torcido muito especial, supondo que a função potencial não tenha pontos críticos. Cao, Sun e Zhang [15] provaram sem condições adicionais que a função potencial de um soliton gradiente de Yamabe tem no máximo um ponto crítico. E quando ela tem um ponto crítico, eles mostraram que o soliton é rotacionalmente si- 
métrico, sem hipóteses adicionais sobre a métrica.

Seja $M^{n}$ uma variedade orientada $n$-dimensional compacta (sem fronteira), e $\mathcal{M}$ o conjunto de estruturas Riemannianas diferenciáveis em $M^{n}$ de volume 1. Dado uma métrica $g \in \mathcal{M}$ definimos o funcional curvatura total, ou funcional Einstein-Hilbert $\mathcal{S}: \mathcal{M} \rightarrow \mathbb{R}$ por

$$
\mathcal{S}(g)=\int_{M} R_{g} d v
$$

onde $R_{g}$ e $d v$ são, respectivamente, a curvatura escalar de $M^{n}$ e o elemento de volume determinado pela métrica e orientação. As métricas que são pontos críticos do funcional curvatura escalar total $\mathcal{S}$ restritos a $\mathcal{M}$ são Einstein; para maiores detalhes, confira o Capítulo 4 em [9].

Por outro lado, a solução do problema de Yamabe mostra que toda variedade Riemanniana compacta $M^{n}$ admite uma métrica de curvatura escalar constante, consideremos o espaço das métricas csc (constant scalar curvature), denominamos tal espaço de $\mathcal{C}=\left\{g \in \mathcal{M} \mid \quad R_{g}\right.$ constant $\}$, em $M^{n}$. Quando restringimos o funcional curvatura escalar total a uma classe de métricas conformes com curvatura escalar constante obtemos um conjunto de pontos crítico. A existência desses pontos críticos é exatamente o problema de Yamabe. Na verdade, um teorema de Koiso [38] mostra que, sob condições genéricas, $\mathcal{C}$ é uma variedade de dimensão infinita (ver p. 127 em [9]). Foi conjecturado que os pontos críticos do funcional curvatura escalar total $\mathcal{S}$ restritos a $\mathcal{C}$ e com volume unitário são métricas de Einstein (cf. [9] p. 128). A métrica CPE (critical point equation) é uma tripla $\left(M^{n}, g, f\right)$ onde $f$ é uma função suave em $M$ que satisfaz a seguinte equação

$$
(1+f) \stackrel{\circ}{R} i c=\nabla^{2} f+\frac{R f}{n(n-1)} g,
$$

onde Ric, $\nabla^{2}=$ Hess e $R$ são, respectivamente o tensor de Ricci, o Hessiano e a curvatura escalar $R$ da métrica $g$, tal que $\stackrel{\circ}{R} i c=R i c-\frac{R}{n} g$.

Nos últimos anos, muitas respostas parciais para conjectura foram obtidas. Por exemplo, Lafontaine [41] provou que a conjectura CPE é verdadeira com a condição da métrica ser localmente conformemente plana e

$$
\operatorname{Ker}\left\{\nabla_{g}^{2} f-\left(\Delta_{g} f\right) g-f \operatorname{Ric}_{g}\right\} \neq 0
$$

Mas em 2011 Chang, Hwang e Yun removeram a condição (2) (cf. [19]). Além disso, Hwang, em [32], considerou a conjectura no caso tridimensional com a hipótese de que a norma do gradiente da função potencial $f$ fosse uma função de apenas $f$; veja também [33]. Em [35], Hwang também mostrou que a conjectura vale se $f \geq-1$. Yun, Chang 
e Hwang [20] provaram que se a variedade com a métrica CPE tem tensor de Ricci paralelo, então ela é isométrica à esfera. A conjectura também foi provada recentemente com a hipótese de que o tensor curvatura seja harmônico [19]. No caso tridimensional a conjectura foi demonstrada por Hwang [34] com apenas a condição de que (2) aconteça. O artigo de Qing-Yuan [37] provou que a conjectura é verdadeira supondo que o tensor de Bach seja plano. Em 2014, Barros e Ribeiro Jr [4] provaram que a conjectura é verdadeira em dimensão 4 supondo que a variedade seja localmente semi conformemente plana.

Iremos estudar algumas propriedades das métricas CPE no Capítulo 4. Precisamente, vamos estudar a norma do gradiente da função potencial. Mostraremos, no Teorema 4.5, que a métrica CPE $n$-dimensional é Einstein se, e somente se,

$$
|\nabla f|^{2}+\frac{R f^{2}}{n(n-1)}=\Lambda
$$

onde $\Lambda$ é constante. O Teorema 4.9, o último resultado desse capítulo, também responde a conjectura CPE supondo que a função

$$
G=|\nabla f|^{2}+\frac{R f^{2}}{n(n-1)}
$$

seja harmônica na fronteira de toda componente conexa do conjunto $M_{0}=\left\{x \in M^{n} ; 1+\right.$ $f(x)<0\}$.

E no Capítulo 5 estudamos a métrica CPE em dimensão quatro. As variedades de dimensão quatro possuem propriedades bem particulares. Com a hipótese do divergente do tensor $W^{+}$ser nulo, ou seja, tensor $W^{+}$harmônico, mostramos que a métrica CPE 4-dimensional é Einstein se todo ponto de $\Sigma_{c}=\left\{p \in M^{4} ; f(p)=c\right\}$, para todo $c \neq-1$ pertencente a $\mathbb{R}$, for umbílico (Teorema 5.10). Em seguida, como corolário da Proposição 5.8 , provamos que a métrica CPE é Einstein supondo que $\delta W^{+}=0$ e

$$
\frac{4}{3} \stackrel{\circ}{\operatorname{Ric}}(N, N)^{2}=|\stackrel{\circ}{\operatorname{Ric}}|^{2}
$$

onde $N=\frac{\nabla f}{|\nabla f|}$. No Teorema 5.12, demonstramos que a métrica CPE, para $n=4$, é Einstein se $\delta W^{+}=0$ e

$$
\int_{M^{4}}|\nabla f|^{2} \stackrel{\circ}{\operatorname{Ric}}(\nabla f, \nabla f)=\frac{R^{2}}{36} \int_{M^{4}} G f^{2} .
$$




\section{Capítulo 1}

\section{Preliminares}

Neste capítulo preliminar, vamos enunciar e demonstrar alguns resultados que serão utilizados nos próximos capítulos.

\subsection{Variedades semi-Riemannianas, métricas confor- mes e Tensores}

Vamos, em primeiro lugar, enunciar alguns fatos sobre formas bilineares.

Se $V$ é um espaço vetorial com $\operatorname{dim} V>0$, uma forma bilinear simétrica sobre $V$ é uma função bilinear $b: V \times V \rightarrow \mathbb{R}$ tal que

$$
b(v, w)=b(w, v) ; \quad \forall \quad v, w \in V .
$$

Definição 1.1. Uma forma bilinear simétrica sobre um espaço vetorial $V$ é:

a) positiva (negativa) definida se $b(v, v)>0(<0), \forall v \in V$.

b) positiva (negativa) semi-definida se $b(v, v) \geq 0(\leq 0), \forall v \in V$.

c) não-degenerada se $b(v, w)=0, \forall w \in V$ implica em $v=0$.

Se $\beta=\left\{e_{1}, \ldots, e_{n}\right\}$ é uma base de $V$, dizemos que a matriz $b_{i j}=b\left(e_{i}, e_{j}\right)$ é chamada a matriz de $b$ relativa à base $\beta$. Uma forma bilinear simétrica é não-degenerada se, e somente se, $b_{i j}$ é inversível.

Definição 1.2. Um tensor métrico $g$ sobre uma $M$ é uma aplicação que associa a cada ponto $p \in M$ uma forma bilinear, simétrica e não-degenerada em $T_{p} M$. 
Definição 1.3. Uma variedade semi-Riemanniana $(M, g)$ é uma variedade diferenciável $M$, munida do tensor métrico $g$.

Para um sistema de coordenadas locais $\left(x_{1}, \ldots, x_{n}\right)$ em uma vizinhança de um ponto $p \in M$, as componentes do tensor métrico $g$ são $g_{i j}=g\left(\frac{\partial}{\partial x_{i}}, \frac{\partial}{\partial x_{j}}\right)$. Como $g$ é nãodegenerado, temos que a matriz $g_{i j}$ é inversível e a sua inversa será denotada por $g^{i j}$.

Definição 1.4. Um vetor tangente $v$ em uma variedade semi-Riemanniana $(M, g)$ é de um dos três tipos:

a) tipo-espaço se $g(v, v)>0$ ou $v=0$.

b) tipo-nulo (ou tipo-luz) se $g(v, v)=0$ e $v \neq 0$.

c) tipo-tempo se $g(v, v)<0$.

Assim como no caso Riemanniano, podemos introduzir os conceitos de conexão e derivada covariante em uma variedade semi-Riemanniana $(M, g)$. E também podemos mostrar que tal conexão é bem-definida e única, assim como no caso Riemanniano. Denotaremos tal conexão por $\nabla$, a conexão de Levi-Civita de $(M, g)$. Tal conexão é caracterizada pela fórmula de Koszul:

$$
\begin{aligned}
2 g\left(\nabla_{V} W, X\right) & =V g(W, X)+W g(X, V)-X g(V, W)-g(V,[W, X]) \\
& +g(W,[X, V])+g(X,[V, W])
\end{aligned}
$$

onde $W, V$ e $X$ pertencem à $\chi(M)$.

Em um sistema de coordenadas $\left(U, x\left(x_{1}, \ldots, x_{n}\right)\right)$, temos que as funções $\Gamma_{i j}^{k}$ definidas em $U$ por

$$
\nabla_{\frac{\partial}{\partial x_{i}}} \frac{\partial}{\partial x_{j}}=\sum_{k} \Gamma_{i j}^{k} \frac{\partial}{\partial x_{k}}
$$

são os símbolos de Christoffel da conexão $\nabla$. Segue pela equação (1.1) que

$$
\Gamma_{i j}^{k}=\frac{1}{2} \sum_{m} g^{m k}\left[\frac{\partial}{\partial x_{i}} g_{j m}+\frac{\partial}{\partial x_{j}} g_{i m}-\frac{\partial}{\partial x_{m}} g_{i j}\right] \text {. }
$$

Os conceitos de transporte paralelo e geodésica seguem de maneira análoga ao caso Riemanniano.

A função $R: \chi(M) \times \chi(M) \times \chi(M) \rightarrow \chi(M)$ dada por

$$
R(X, Y) Z=\nabla_{Y} \nabla_{X} Z-\nabla_{X} \nabla_{Y} Z+\nabla_{[X, Y]} Z
$$


onde $\nabla$ é a conexão de Levi-Civita, é multilinear e será chamada de tensor curvatura de $M$.

Um subespaço bi-dimensional $\sigma$ do espaço tangente $T_{p} M$ é chamado plano tangente a $M$ em $p$. Para vetores tangentes $X, Y$ definimos

$$
Q(X, Y)=g(X, X) g(Y, Y)-g(X, Y)^{2}
$$

Um plano tangente $\sigma$ é não-degenerado se, e somente se, $Q(X, Y) \neq 0$ para uma base qualquer $X, Y$ de $\sigma$. Assim, temos o seguinte lema

Lema 1.5. Seja $\sigma$ um plano tangente não-degenerada de $M$ em p. O número

$$
K(X, Y)=\frac{g(R(X, Y) X, Y)}{Q(X, Y)}
$$

é independente da escolha da base $X, Y$ para $\sigma$ e é chamado de curvatura seccional de $\sigma$ em $p$.

Definição 1.6. Seja $R$ o tensor curvatura de $(M, g)$. O tensor curvatura de Ricci é definido por

$$
\operatorname{Ric}_{p}(X, Y)=\operatorname{traço}\{Z \rightarrow R(X, Z) Y\}
$$

onde $X, Y$ e $Z \in T_{p} M$.

Em um sistema de coordenadas,

$$
R_{i k}=g^{j l} R_{i j k l}
$$

Sabemos que, para cada ponto $p \in M$,

$$
\operatorname{Ric}_{p}: T_{p} M \times T_{p} M \rightarrow \mathbb{R}
$$

é uma forma bilinear e simétrica. Então, considerando $A: T_{p} M \rightarrow T_{p} M$ a aplicação linear auto-adjunta associada ao tensor $\operatorname{Ric}_{p}$, definimos

Definição 1.7. Seja $(M, g)$ uma variedade semi-Riemanniana. A curvatura escalar $R$ de $M$ é uma função $R: M \rightarrow \mathbb{R}$, definida por $R(p)=\operatorname{contração~}\left(R i c_{p}\right)$. Em um sistema de coordenadas

$$
R=\sum_{i, j} g^{i j} R_{i j}
$$


Considerando $f$ uma função diferenciável introduzimos em seguida, os conceitos de gradiente, hessiano e laplaciano da função $f$.

Definição 1.8. Seja $(M, g)$ uma variedade semi-Riemanniana e $f$ uma função diferenciável. Então

a) o gradiente de $f$, denotado por $\nabla f$, é dado por

$$
g(\nabla f, X)=d f(X) ; \forall X \in \chi(M)
$$

b) a hessiana de $f$ é dada por

$$
\nabla^{2} f(X, Y)=\operatorname{Hessf}(X, Y)=g\left(\nabla_{X} \nabla f, Y\right)
$$

c) e o laplaciano de $f$ é

$$
\Delta f=\operatorname{div}(\nabla f)
$$

onde div $: \chi(M) \rightarrow \mathbb{R}$ é dado por

$$
\operatorname{div}(X)=\operatorname{contração}\left\{Y \rightarrow \nabla_{Y} X\right\}
$$

Vamos expor alguns resultados sobre métricas conformes que serão muito utilizados ao longo do texto.

Proposição 1.9. [9] Seja $(M, g)$ uma variedade semi-Riemanniana de dimensão $n \geq 3$ e $\bar{g}=\frac{g}{\varphi^{2}}$ uma métrica conforme à $g$. Então, os tensores de Ricci de g e de $\bar{g}$ satisfazem a relação

$$
\operatorname{Ric}_{\bar{g}}-\operatorname{Ric}_{g}=\frac{1}{\varphi^{2}}\left\{(n-2) \varphi \operatorname{Hess}_{g}(\varphi)+\left[\varphi \Delta_{g} \varphi-(n-1)\left|\nabla_{g} \varphi\right|^{2}\right] g\right\}
$$

Portanto, temos que

$$
R_{\bar{g}}=\sum_{k=1}^{n} \varepsilon_{k} \varphi^{2}\left(\operatorname{Ric}_{\bar{g}}\right)_{k k}=(n-1)\left(2 \varphi \Delta_{g} \varphi-n\left|\nabla_{g} \varphi\right|^{2}\right)
$$

Lema 1.10. Seja $(M, g)$ uma variedade Riemanniana. Então a conexão de Levi-Civita 
$\bar{\nabla}$ para uma métrica conforme $\bar{g}=\frac{g}{\varphi^{2}}$ satisfaz

$$
\bar{\nabla}_{X} Y=\nabla_{X} Y-\frac{1}{\varphi}[(X \varphi) Y+(Y \varphi) X-g(X, X) \nabla \varphi]
$$

Demonstração. Utilizando a fórmula de Koszul (1.1), uma conta direta nos dá o resultado.

$$
\begin{aligned}
\frac{2}{\varphi^{2}} g\left(\bar{\nabla}_{X} Y, Z\right) & =Y\left(\frac{1}{\varphi^{2}} g(X, Z)\right)+X\left(\frac{1}{\varphi^{2}} g(Y, Z)\right)-Y\left(\frac{1}{\varphi^{2}} g(X, Z)\right) \\
& -\left(\frac{1}{\varphi^{2}} g(Y,[X, Z])\right)-\left(\frac{1}{\varphi^{2}} g(X,[Y, Z])\right)-\left(\frac{1}{\varphi^{2}} g(Z,[X, Y])\right) .
\end{aligned}
$$

Expandindo a expressão anterior temos que

$$
\begin{aligned}
\frac{2}{\varphi^{2}} g\left(\bar{\nabla}_{X} Y, Z\right)= & -\frac{2}{\varphi^{3}}(Y \varphi) g(X, Z)+\frac{1}{\varphi^{2}}\left[g\left(\nabla_{Y} X, Z\right)+g\left(X, \nabla_{Y} Z\right)\right] \\
& -\frac{2}{\varphi^{3}}(X \varphi) g(Y, Z)+\frac{1}{\varphi^{2}}\left[g\left(\nabla_{X} Y, Z\right)+g\left(Y, \nabla_{X} Z\right)\right] \\
& +\frac{2}{\varphi^{3}}(Z \varphi) g(X, Y)+\frac{1}{\varphi^{2}}\left[g\left(\nabla_{Z} X, Y\right)+g\left(X, \nabla_{Z} Y\right)\right] \\
& -\frac{1}{\varphi^{2}}(g(Y,[X, Z])+g(X,[Y, Z])+g(Z,[X, Y]))
\end{aligned}
$$

Podemos simplificar a expressão anterior utilizando a definição de colchete,

$$
g\left(\bar{\nabla}_{X} Y, Z\right)=g\left(\nabla_{X} Y, Z\right)-\frac{1}{\varphi}[(X \varphi) g(Y, Z)+(Y \varphi) g(X, Z)-g(X, X) g(\nabla \varphi, Z)]
$$

donde segue o resultado.

Ao longo desta seção, vamos relembrar alguns fatos básicos sobre tensores que serão úteis na demonstração dos nossos resultados.

Vale ressaltar que ao longo do texto usaremos a notação de Einstein para soma, a menos que seja mencionado o contrário. De acordo com esta convenção, quando uma variável de índice aparece duas vezes em um único termo, uma vez em um (sobrescrita) superior e uma vez em uma posição inferior (subscrito), isso implica que estamos somando sobre todos os seus possíveis valores. Vejamos como exemplo, como podem ser escritas as entradas do gradiente de uma função definida numa variedade $M^{n}$,

$$
\nabla_{i} f=g_{i j} \nabla^{j} f
$$

veja que a soma ocorreu sobre o índice $j$. 
Vamos utilizar, ao longo do texto, uma notação muito importante sobre o tensor curvatura (e conseqüentemente, essa notação recairá sobre os tensores de Weyl, Cotton e Ricci). Considere um sistema de coordenadas locais $\left(x_{1}, \ldots, x_{n}\right)$, tome uma base $\left\{e_{i}=\right.$ $\left.\frac{\partial}{\partial x_{i}}\right\}_{i=1}^{n}$ associada ao sistema de coordenadas locais, temos que

$$
R\left(e_{i}, e_{j}, e_{k}, \nabla f\right)=R_{i j k l} \nabla^{l} f
$$

De fato, lembrando que

$$
\nabla f=\left(g^{i j} \nabla_{i} f\right) e_{j}=\left(\nabla^{j} f\right) e_{j}
$$

Temos, pelo fato do tensor curvatura ser multilinear,

$$
\begin{aligned}
R\left(e_{i}, e_{j}, e_{k}, \nabla f\right) & =R\left(e_{i}, e_{j}, e_{k},\left(g^{s l} \nabla_{s} f\right) e_{l}\right)=R\left(e_{i}, e_{j}, e_{k},\left(\nabla^{l} f\right) e_{l}\right) \\
& =R\left(e_{i}, e_{j}, e_{k}, e_{l}\right)\left(\nabla^{l} f\right)=R_{i j k l} \nabla^{l} f .
\end{aligned}
$$

Para os operadores $S, T: \mathcal{H} \rightarrow \mathcal{H}$ definidos num espaço de Hilbert $n$-dimensional $\mathcal{H}$ o produto interno Hilbert-Schmidt é definido por

$$
\langle S, T\rangle=\operatorname{tr}\left(\mathrm{ST}^{\star}\right)
$$

onde $\operatorname{tr}$ e $\star$ são, respectivamente, o traço e a operação adjunto. Além disso, se $I$ é o operador identidade em $\mathcal{H}$ o operador sem traço de $T$ é dado por

$$
\stackrel{\circ}{T}=T-\frac{\operatorname{tr} T}{n} I .
$$

Em particular a norma de $\stackrel{\circ}{T}$ satisfaz

$$
|\stackrel{\circ}{T}|^{2}=|T|^{2}-\frac{(\operatorname{tr} T)^{2}}{n}
$$

Agora, nós vamos lembrar que para uma variedade Riemanniana $\left(M^{n}, g\right), n \geq 3$, o tensor de Weyl $W$ é definido pela seguinte fórmula

$$
\begin{aligned}
R_{i j k l}= & W_{i j k l}+\frac{1}{n-2}\left(R_{i k} g_{j l}+R_{j l} g_{i k}-R_{i l} g_{j k}-R_{j k} g_{i l}\right) \\
& -\frac{R}{(n-1)(n-2)}\left(g_{j l} g_{i k}-g_{i l} g_{j k}\right)
\end{aligned}
$$

onde $R_{i j k l}$ é o operador curvatura. Sabemos que $W=0$ para $n=3$. Em dimensão $n=4$ uma variedade é localmente conformemente plana se, e somente se, $W=0$. A equação 
de Ricci é dada por

$$
\nabla_{i} \nabla_{j} \nabla_{k} f-\nabla_{j} \nabla_{i} \nabla_{k} f=R_{i j k s} \nabla^{s} f
$$

onde $f: M \rightarrow \mathbb{R}$ é uma função diferenciável.

Definimos o tensor de Ricci sem traço por

$$
\stackrel{\circ}{R} i c=R i c-\frac{R}{n} g
$$

onde $R$ é a curvatura escalar do tensor métrico $g$.

O tensor de Cotton $C$ é

$$
C_{i j k}=\nabla_{i} R_{j k}-\nabla_{j} R_{i k}-\frac{1}{2(n-1)}\left(\nabla_{i} R g_{j k}-\nabla_{j} R g_{i k}\right) .
$$

Esses dois tensores estão relacionados da seguinte maneira

$$
C_{i j k}=-\frac{(n-2)}{(n-3)} \nabla^{l} W_{i j k l}=\frac{(n-2)}{(n-3)} g^{s l} \nabla_{s} W_{l k i j}
$$

para $n \geq 4$. Para maiores detalhes sobre esses tensores recomendamos, por exemplo, [9].

Em [14], Cao e Chen definiram o tensor $D$ que é a ponte entre o tensor de Weyl e os solitons quasi Yamabe gradiente (Proposição 3.2 a seguir). Definiremos o 3-tensor $D$ por

$$
\begin{aligned}
D_{i j k} & =\frac{1}{n-2}\left(R_{j k} \nabla_{i} f-R_{i k} \nabla_{j} f\right)+\frac{1}{(n-1)(n-2)}\left(R_{i l} \nabla^{l} f g_{j k}-R_{j l} \nabla^{l} f g_{i k}\right) \\
& -\frac{R}{(n-1)(n-2)}\left(g_{j k} \nabla_{i} f-g_{i k} \nabla_{j} f\right) .
\end{aligned}
$$

Esse tensor $D$ é anti-simétrico nos dois primeiros índices e livre de traço, isto é,

$$
D_{i j k}=-D_{j i k} \quad \text { e } \quad g^{i j} D_{i j k}=g^{i k} D_{i j k}=g^{j k} D_{i j k}=0
$$

\subsection{Teorema de Stokes}

Nessa seção, nós vamos ver um resultado central na teoria de integração em variedades: O teorema de Stokes. Este teorema é uma generalização do teorema fundamental 
do cálculo (ver [42]). Antes de falarmos sobre o teorema, vamos definir o que é uma variedade com fronteira.

Definição 1.11. Uma carta de fronteira $\varphi: U \rightarrow V$ para um espaço topológico $M$ sobre um ponto $x \in M$ é uma aplicação contínua de um aberto $U \subset M$ para um subconjunto aberto $V$ de $\mathbb{R}_{+}^{n}=\left\{\left(x_{1}, \ldots, x_{n}\right): x_{n} \geq 0\right\} \operatorname{com} \varphi(x) \in \mathbb{R}^{n-1} \times\{0\}$.

Onde os subconjuntos de $\mathbb{R}_{+}^{n}$ são conjuntos da forma $W \bigcap \mathbb{R}_{+}^{n}$ onde $W \subset \mathbb{R}^{n}$ é aberto.

Definição 1.12. Um atlas de fronteira $\mathcal{A}$ diferenciável para um espaço topológico $M$ é uma coleção de aplicações $\varphi_{\alpha}: U_{\alpha} \rightarrow V_{\alpha}$ onde cada aplicação é uma carta, ou uma carta de fronteira, para $M$, tal que $\bigcup U_{\alpha}=M$ e $\varphi_{\alpha} \circ \varphi_{\beta}^{-1}$ é uma aplicação diferenciável entre conjuntos abertos de $\mathbb{R}_{+}^{n}$ para cada $\alpha$ e $\beta$.

Definição 1.13. Uma variedade diferenciável com fronteira é um espaço topológico $M$ equipado com uma classe de equivalência de atlas de fronteira diferenciável, onde dois atlas de fronteira são equivalentes se sua união é um atlas de fronteira.

Se $M$ é uma variedade com fronteira, então a fronteira $\partial M$ de $M$ é o subconjunto de todos os pontos $x \in M$ tais que para cada $x$ existe uma carta de fronteira.

Proposição 1.14. Se $M$ é uma variedade diferenciável de $n$-dimensional com fronteira, então วM é uma variedade diferenciável $(n-1)$-dimensional, com atlas dado pela restrição de $\partial M$ das cartas de fronteiras de $M$.

Suponha que $M^{n}$ é uma variedade diferenciável com fronteira orientada. Isto é, $M$ está equipada com um atlas de fronteira diferenciável $\mathcal{A}$ tal que $\varphi_{\alpha} \circ \varphi_{\beta}^{-1}$ é uma aplicação que preserva orientação, para cada $\alpha$ e $\beta$.

Proposição 1.15. $\partial M$ é uma variedade diferenciável orientada com dimensão $n-1$.

A orientação construída em $\partial M$ é chamada de orientação induzida em $\partial M$ pela orientação de $M$.

Teorema 1.16. Seja $M$ uma variedade diferenciável com fronteira de dimensão n, e $\omega$ uma $(n-1)$-forma diferenciável em $\partial M$. Então

$$
\int_{M} d \omega=\int_{\partial M} \omega
$$

Repare que $\partial M$ tem a orientação induzida por $M$, e $\omega$ é restrita a $\partial M$ no lado direito da equação acima. 
Corolário 1.17. Seja $M$ uma variedade diferenciável compacta sem fronteira. Então

$$
\int_{M} d \omega=0
$$

Corolário 1.18. Seja $M$ é uma variedade diferenciável compacta com fronteira. Se $\omega$ é uma forma fechada sobre $M$, então:

$$
\int_{\partial M} \omega=0 .
$$

\subsection{Teoria dos grupos de simetria}

Um grupo de Lie é um grupo de simetria de um sistema de equações diferenciais, quando, a ação do grupo transforma soluções do sistema em outras soluções. A aplicação mais importante, consiste no uso de invariantes pela ação do grupo (ou de subgrupos) para reduzir o número de variáveis de uma equação diferencial parcial, podendo até mesmo, reduzir para um sistema de equações diferenciais ordinárias (veja [51]).

Definição 1.19. Seja $M$ uma variedade diferenciável. Um grupo local de transformações agindo em $M$ é dado por um grupo de Lie $G$, um subconjunto aberto $U$, tal que

$$
\{e\} \times M \subset U \subset G \times M,
$$

onde e é o elemento neutro do grupo, e uma aplicação diferenciável $\psi: U \rightarrow M$ satisfazendo as seguintes propriedades:

a)Se $(h, x),(g, \psi(h, x))$ e $(g \cdot h, x)$ pertencem a $U$, então

$$
\psi(g, \psi(h, x))=\psi(g \cdot h, x)
$$

b)Para todo $x \in M$,

$$
\psi(e, x)=x
$$

c)Se $(g, x) \in U$, então $\left(g^{-1}, \psi(g, x)\right) \in U$ e

$$
\psi\left(g^{-1}, \psi(g, x)\right)=x
$$


Simplificando, denote $\psi(g, x)=g \cdot x$. Assim, teremos que

$$
\begin{aligned}
g \cdot(h \cdot x) & =(g \cdot h) \cdot x, \\
e \cdot x & =x, \\
g^{-1} \cdot(g \cdot x) & =x,
\end{aligned}
$$

onde $g, h \in G$ e $x \in M$ estão nas condições da definição.

Definição 1.20. Seja $G$ um Grupo de Lie local de transformações agindo em $M$, então, para cada $x \in M$, definimos a órbita através de $x$ por

$$
\mathcal{O}_{x}=\left\{g_{1} \cdot g_{2} \cdot \ldots \cdot g_{k} \cdot x \mid k \geq 1, g_{i} \in G \quad \text { e } \quad g_{1} \cdot g_{2} \cdot \ldots \cdot g_{k} \cdot x \quad \text { está definido }\right\}
$$

Além disso, dizemos que o grupo $G$ age semi-regularmente se todas as órbitas são subvariedades de $M$ com a mesma dimensão.

Exemplo 1.21. Exemplo de Grupos de Transformações.

a) O grupo de translações em $\mathbb{R}^{n}$ : Seja $v \neq 0$ um vetor fixado em $\mathbb{R}^{n}$ e seja $G=\mathbb{R}$ o grupo aditivo. Defina

$$
\psi_{v}(\varepsilon, x)=x+\varepsilon v, \quad x \in \mathbb{R}^{n}, \quad \varepsilon \in \mathbb{R} .
$$

As órbitas, nesse caso, são retas paralelas a $v$ de forma que a ação é semi-regular com órbitas unidimensionais.

b) O grupo de dilatações em $\mathbb{R}^{n}$ : Considere novamente o grupo aditivo $G=\mathbb{R}$, uma constante $\lambda>0$ e defina

$$
\psi_{\lambda}(\varepsilon, x)=\lambda^{\varepsilon} x, \quad x \in \mathbb{R}^{n}, \quad \varepsilon \in \mathbb{R}
$$

Nesse exemplo temos órbitas unidimensionais, quando $x \neq 0$ e a órbita singular consistindo apenas da origem $\{0\}$. Dessa forma, a ação é semi-regular no conjunto aberto $\mathbb{R}^{n} \backslash\{0\}$.

Definição 1.22. Seja $G$ um grupo local de transformações agindo em uma variedade $M$. Uma função $\xi: M \rightarrow \mathbb{R}$ é chamada um invariante de $G$ se para todo $x \in M$ e para todo $g \in G$ tal que $g \cdot x$ está definido, vale

$$
\xi(g \cdot x)=\xi(x)
$$

Proposição 1.23. Seja $G$ um grupo conexo de transformações agindo em uma variedade 
M. Uma função $\xi: M \rightarrow \mathbb{R}$ é uma função invariante para $G$ se, e somente se,

$$
v(\xi)=0, \quad \forall x \in M
$$

e para todo gerador infinitesimal $v$ de $G$.

Definição 1.24. Considere $\xi_{1}(x), \ldots, \xi_{k}(x)$ funções reais e diferenciáveis, definidas em M. Então

a) $\xi_{1}, \ldots, \xi_{k}$ são chamadas funcionalmente dependentes se para cada $x \in M$ existe uma vizinhança $U$ de $x$ e uma função real diferenciável $F\left(z_{1}, \ldots, z_{k}\right)$, não identicamente nula em qualquer subconjunto de $\mathbb{R}^{k}$, tal que

$$
F\left(\xi_{1}(x), \ldots, \xi_{k}(x)\right)=0
$$

para todo $x \in U$.

b) $\xi_{1}, \ldots, \xi_{k}$ são chamadas funcionalmente independentes se não são funcionalmente dependentes quando restritas a um subconjunto aberto qualquer $U \subset M$.

O seguinte teorema nos dá a quantidade máxima de invariantes funcionalmente independentes que podemos obter por uma ação de $G$ em $M$ :

Teorema 1.25. Suponha que $G$ age semi-regularmente na variedade $M$ de dimensão $m$ com órbitas $s$-dimensionais. Se $x_{0} \in M$, então existem precisamente $m-s$ invariantes funcionalmente independentes $\xi_{1}, \ldots, \xi_{m-s}$ definidos em uma vizinhança de $x_{0}$. Além disso, qualquer outro invariante definido nesta vizinhança é da forma

$$
\xi(x)=F\left(\xi_{1}, \ldots, \xi_{m-s}\right)
$$

para uma função diferenciável $F$.

Vamos mostrar como encontrar invariantes de um dado grupo de transformações. Inicialmente, suponha que $G$ é um grupo de transformações a 1-parâmetro agindo em $M$, com gerador infinitesimal

$$
v=\eta_{1}(x) \partial_{x_{1}}+\ldots+\eta_{m}(x) \partial_{x_{m}}
$$

expresso em alguma parametrização local dada. Pela equação (1.14), um invariante $\xi$ de $G$ é uma solução da seguinte equação diferencial parcial linear de primeira ordem

$$
v(\xi)=\eta_{1}(x) \partial_{x_{1}}(\xi)+\ldots+\eta_{m}(x) \partial_{x_{m}}(\xi)=0
$$


O teorema (1.25) diz então que, se $v$ é não nulo, então existem $m-1$ invariantes funcionalmente independentes, consequentemente, $m-1$ soluções funcionalmente independentes da equação diferencial parcial (1.15) em uma vizinhança de $x_{0} \in M$.

O cálculo de invariantes independentes para grupo de transformações a $r$-parâmetros consiste no seguinte: Se $v_{k}=\sum_{i} \eta_{i}^{k} \partial_{x_{i}}, k=1, \ldots, r$ forma uma base para os geradores infinitesimais, então os invariantes são encontrados resolvendo o seguinte sistema linear homogêneo de equações diferenciais parciais de primeira ordem

$$
v_{k}(\xi)=\sum_{i=1}^{m} \eta_{i}^{k} \partial_{x_{i}} \xi=0, \quad k=1, \ldots, r
$$

Uma das vantagens em se encontrar soluções invariantes pela ação de um grupo de simetria é que esse método nos permite reduzir a quantidade de variáveis do sistema de equações diferenciais parciais, podendo inclusive reduzi-lo a uma equação diferencial ordinária que possui mais métodos para encontrar soluções.

\subsection{Soliton gradiente quasi Yamabe}

O fluxo de Yamabe,

$$
\frac{\partial}{\partial t} g(t)=-R g(t)
$$

foi introduzido por R.Hamilton na tentativa de resolver o problema de Yamabe (ver [29]). Solitons são ferramentas importantes quando estamos interessados em entender a geometria do fluxo já que os solitons podem aparecer como modelos de singularidades. Solitons de Yamabe representam um tipo de solução para o fluxo de Yamabe.

Definição 1.26. Uma variedade Riemanniana $\left(M^{n}, g\right)$ de dimensão $n \geq 3$ é chamada de soliton gradiente de Yamabe se existe uma função potencial diferenciável $f: M^{n} \rightarrow \mathbb{R}$ e uma constante $\lambda$ tal que

$$
(R-\lambda) g_{i j}=\nabla_{i} \nabla_{j} f
$$

Se $\lambda>0, \lambda=0$ ou $\lambda<0$, teremos, respectivamente, um soliton gradiente de Yamabe contraído, estável ou expansivo.

Motivados pelos resultados das variedades quasi Einstein (veja [17]), a teoria dos 
solitons gradientes quasi Yamabe começou a ser investigada. Métricas quasi Einstein generalizam os solitons de Ricci. Essas métricas quasi Einstein são usadas quando estamos estudando variedades com peso (ver [58]). Os solitons gradientes quasi Yamabe são generalizações dos solitons de Yamabe gradiente.

Definição 1.27. Um soliton gradiente quasi Yamabe $\left(M^{n}, g, f\right)$, onde $\left(M^{n}, g\right)$ é uma variedade Riemanniana de dimensão $n \geq 3$ com função potencial diferenciável $f: M^{n} \rightarrow$ $\mathbb{R}$ e duas constantes $\lambda, m(m \neq 0)$ que satisfazem

$$
(R-\lambda) g_{i j}=\nabla_{i} \nabla_{j} f-\frac{1}{m} \nabla_{i} f \nabla_{j} f
$$

Repare que quando $m \rightarrow \infty$ então (1.17) se reduz a (1.16) e se $f$ é constante, dizemos que o soliton gradiente quasi Yamabe é trivial.

Adiante, vamos enunciar alguns resultados sobre (quasi) solitons gradientes de Yamabe que serão explorados ao longo do texto.

Teorema 1.28. [30] Seja $\left(M^{4}, g, f\right)$ um soliton gradiente quasi Yamabe completo não trivial satisfazendo (1.17) com curvatura seccional positiva e $D_{i j k}=0$. Então $\left(M^{4}, g, f\right)$ é rotacionalmente simétrico.

Teorema 1.29. [15] Seja $\left(M^{n}, g, f\right)$ um soliton gradiente de Yamabe completo não trivial satisfazendo (1.16). Então $|\nabla f|^{2}$ é constante nos conjuntos de nível de $f$, e $f$ satisfaz uma das seguintes propriedades:

(i) f tem um único ponto crítico $x_{0} \in M^{n}, e\left(M^{n}, g, f\right)$ é rotacionalmente simétrico e igual ao produto torcido

$$
\left([0,+\infty), d r^{2}\right) \times|\nabla f|\left(\mathbb{S}^{n-1}, \bar{g}_{c a n}\right),
$$

onde $\bar{g}_{\text {can }}$ é a métrica canônica em $\mathbb{S}^{n-1}$, ou

(ii) f não tem ponto críticos e $\left(M^{n}, g, f\right)$ é o produto torcido

$$
\left(\mathbb{R}, d r^{2}\right) \times|\nabla f|\left(N^{n-1}, \bar{g}\right)
$$

onde $\left(N^{n-1}, \bar{g}\right)$ é uma variedade Riemanniana de curvatura escalar constante.

Quando $\left(M^{n}, g, f\right)$ é localmente conformemente plana, temos o seguinte:

Teorema 1.30. [15] Seja $\left(M^{n}, g, f\right)$ um soliton gradiente de Yamabe completo e não trivial satisfazendo a equação (1.16). Suponha que $f$ não tem pontos críticos e que ela é 
localmente conformemente plana. Então $\left(M^{n}, g, f\right)$ é o produto torcido

$$
\left(\mathbb{R}, d r^{2}\right) \times_{|\nabla f|}\left(N^{n-1}, \bar{g}\right)
$$

onde $\left(N^{n-1}, \bar{g}\right)$ espaço de curvatura seccional constante.

Observação 1.31. Vale dizer que os solitons gradientes quasi Yamabe compactos, assim como os solitons gradientes de Yamabe compactos, tem curvatura escalar constante (veja por exemplo $[25,30])$.

\subsection{Estrutura do produto torcido}

Aqui, vamos voltar nossa atenção para uma classe de métricas sobre a variedade produto $B \times F$. Vamos definir o produto torcido (veja [9] e [50]).

Definição 1.32. Sejam $\left(B, g_{B}\right)$ e $\left(F, g_{F}\right)$ variedades Riemannianas e $f>0$ uma função sobre $B$. O produto torcido $M=B \times{ }_{f} F$ é a variedade produto $B \times F$ com a métrica

$$
g=\pi^{*} g_{B}+\left(\pi^{*} f\right)^{2} \sigma^{*} g_{F},
$$

onde $\pi$ e $\sigma$ são as projeções de $B \times F$ em $B$ e $F$, respectivamente. Explicitamente, para $u, v \in T_{(p, q)} M$, temos

$$
g(u, v)=g_{B}(d \pi(u), d \pi(v))+(f \circ \pi)^{2} g_{F}(d \sigma(u), d \sigma(v))
$$

Observação 1.33. Se $f$ é constante igual a 1 , dizemos que $M$ é o produto Riemanniano e $g$ a métrica produto. Quando uma variedade $M$ não pode ser escrita como o produto Riemanniano de outras duas variedades dizemos que ela é irredutível.

As fibras $p \times F=\pi^{-1}(p)$ e as folhas $B \times q=\sigma^{-1}(q)$, com $p \in B$ e $q \in F$, são subvariedades de $M$. A métrica produto torcido é caracterizada por

1. Para cada $q \in F$, a aplicação $\pi \mid(B \times q)$ é uma isometria sobre $B$,

2. Para cada $p \in B$, a aplicação $\sigma \mid(p \times F)$ é uma homotetia sobre $F$, com fator escalar $1 / f(p)$.

3. Para cada $(p, q) \in M$, as folhas $B \times q$ e as fibras $p \times F$ são ortogonais em $(p, q)$, assim podemos decompor $T_{(p, q)} M$ na soma direta

$$
T_{(p, q)} M=T_{(p, q)}(B \times q) \oplus T_{(p, q)}(p \times F) .
$$


Chamaremos os vetores tangentes às folhas de horizontais, e os tangentes às fibras de verticais. Se $v \in T_{(p, q)} M$ denotaremos por $h o r(v)$ e $\operatorname{ver}(v)$ as componentes horizontal e vertical de $v$, respectivamente.

Observação 1.34. Para uma variedade produto $B \times F$, denotando por $\mathfrak{F}(B)$ o conjunto das funções diferenciáveis sobre $B$, temos as seguintes noções de levantamento.

1. Se $h \in \mathfrak{F}(B)$, o levantamento de $h$ para $B \times F$ é $\tilde{h}=h \circ \pi \in \mathfrak{F}(B \times F)$.

2. Se $v \in T_{p} B$ e $q \in F$ então o levantamento $\tilde{v}$ de $v$ em $(p, q)$ é o único vetor em $T_{(p, q)}(B \times q)$, tal que $d \pi(\tilde{v})=v$.

3. Se $X \in \mathfrak{X}(B)$ o levantamento de $X$ para $B \times F$ é o único campo vetorial $\tilde{X}$ cujo valor em cada ponto $(p, q)$ é o levantamento de $X(p)$ para $(p, q)$. Este campo é diferenciável e é o único elemento de $\mathfrak{X}(B \times F)$, tal que $d \pi(\tilde{X})=X$ e $d \sigma(\tilde{X})=0$. Denotamos por $\mathfrak{L}(B)$ o conjunto dos levantamentos dos elementos de $\mathfrak{X}(B)$ para $B \times F$.

Funções, vetores tangentes e campos diferenciáveis sobre $F$ podem ser levantados para $B \times F$ de maneira similar usando a projeção $\sigma$.

A seguir, vamos enunciar alguns resultados sobre o produto torcido que serão muito importantes ao longo do texto. Esses resultados, podem ser encontrados em [50].

Lema 1.35. Se $h \in \mathfrak{F}(B)$, então o gradiente do levantamento $h \circ \pi$ de $h$ para $M=B \times{ }_{f} F$ é o levantamento para $M$ do gradiente de $h$ sobre $B$.

Denotando as conexões Riemannianas de $M, B$ e $F$ por $\nabla, \nabla^{B}$ e $\nabla^{F}$, respectivamente, podemos relacioná-las da seguinte maneira:

Proposição 1.36. Seja $M=B \times_{f} F$ um produto torcido. Se $X, Y \in \mathfrak{L}(B)$ e $V, W \in$ $\mathfrak{L}(F)$, ent $\tilde{a} o$

1. $\nabla_{X} Y \in \mathfrak{L}(B)$ é o levantamento de $\nabla_{X}^{B} Y$ a partir de $B$,

2. $\nabla_{X} V=\nabla_{V} X=\frac{X(f)}{f} V$,

3. $h o r\left(\nabla_{V} W\right)=-\frac{\langle V, W\rangle}{f} \nabla f$,

4. $\operatorname{ver}\left(\nabla_{V} W\right) \in \mathfrak{L}(F)$ é o levantamento de $\nabla_{V}^{F} W$ a partir de $F$,

onde $\nabla f$ é o gradiente de $f$ na métrica $g$. 
Apresentaremos agora um resultado que relaciona as curvaturas de $M$ com as curvaturas da base $B$, da folha $F$.

Proposição 1.37. Seja $M=B \times_{f} F$ um produto torcido com o tensor curvatura $R$. Sejam $R^{B}$ e $R^{F}$ os pullback dos tensores curvatura de $B$ e F, respectivamente. Se $X, Y, Z \in \mathfrak{L}(B)$ e $U, V, W \in \mathfrak{L}(F)$, então

1. $R(X, Y) Z \in \mathfrak{L}(M)$ é o levantamento de $R^{B}(X, Y) Z \in \mathfrak{L}(B)$ a partir de $B$,

2. $R(V, X) Y=\frac{\nabla^{2} f(X, Y)}{f} V$, onde $\nabla^{2}$ é a hessiana do produto torcido $M$, que coincide com a hessiana de $B$ nos vetores horizontais,

3. $R(X, Y) V=R(V, W) X=0$,

4. $R(X, V) W=\frac{\langle V, W\rangle}{f} \nabla_{X} \nabla f$

5. $R(U, V) W=R^{F}(U, V) W-\frac{\langle\nabla f, \nabla f\rangle}{f^{2}}\{\langle W, U\rangle V-\langle W, V\rangle U\}$.

Como conseqüência do resultado acima, vamos mostrar como é o tensor de Ricci do produto torcido, Ric. Denotaremos $\operatorname{Ric}^{B}$ e $R i c^{F}$ o pullback dos tensores de Ricci de $B$ e $F$, respectivamente.

Corolário 1.38. Sobre um produto torcido $M=B \times_{f} F \operatorname{com} n=\operatorname{dim}(F)>1$, se $X, Y$ são horizontais e $V, W$ verticais, então

1. $\operatorname{Ric}(X, Y)=\operatorname{Ric}^{B}(X, Y)-\frac{n}{f} \nabla^{2} f(X, Y)$,

2. $\operatorname{Ric}(X, V)=0$,

3. $\operatorname{Ric}(V, W)=\operatorname{Ric}^{F}(V, W)-\langle V, W\rangle\left\{\frac{\Delta_{B} f}{f}+\frac{1}{f^{2}}(n-1)\langle\nabla f, \nabla f\rangle\right\}$,

onde $\Delta_{B} f$ é o laplaciano de $f$ sobre $B$.

As demonstrações dos resultados citados acima, podem ser encontrados em [9] e [50].

\subsection{Variedades de dimensão 4 e suas propriedades es- peciais}

As variedades de dimensão $n=4$ possuem propriedades muito especiais. Vamos introduzir alguns conceitos já conhecidos que podem ser encontrados na literatura ([9], [39] e [26]). 
O fibrado das 2-formas em uma variedade Riemanniana orientada compacta 4dimensional pode ser invariantemente decomposto em soma direta; fatos importantes sobre esse assunto podem ser encontrados em [9] e [54]. Por exemplo, em uma variedade Riemannina $\left(M^{4}, g\right)$, o tensor de Weyl $W$ é um endomorfismo do fibrado das 2-formas $\Lambda^{2}=\Lambda_{+}^{2} \oplus \Lambda_{-}^{2}$ tal que

$$
W=W^{+} \oplus W^{-}
$$

onde $W^{ \pm}: \Lambda_{ \pm}^{2} \longrightarrow \Lambda_{ \pm}^{2}$ são chamados, respectivamente, de self-dual e anti-self dual partes de $W$. Métricas semi localmente conformemente planas são também conhecidas como self-dual tensor de Weyl ou anti-self dual tensor de Weyl se $W^{-}=0$ ou $W^{+}=0$, respectivamente. Vale lembrar que uma variedade Riemanniana, de dimensão $n \geq 4$, é localmente conformemente plana se, e somente se, o seu tensor de Weyl é identicamente nulo.

No que se segue, denotaremos uma variedade 4-dimensional orientada $M^{4}$ e $g$ como sua métrica Riemanniana. Como mencionamos anteriormente as 4-variedades são bastante especiais. Por exemplo, seguindo a notação usada em [26] (ver também [56] p. 46), dado um referencial ortogonal local $\left\{e_{1}, e_{2}, e_{3}, e_{4}\right\}$ num aberto de $M^{4}$ com base dual associada $\left\{e^{1}, e^{2}, e^{3}, e^{4}\right\}$, existe um único operador $*$ chamado de estrela de Hodge (agindo nos bivetores), tal que

$$
\begin{aligned}
& *\left(e^{1} \wedge e^{2}\right)=e^{3} \wedge e^{4} \\
& *\left(e^{1} \wedge e^{3}\right)=e^{4} \wedge e^{2} \\
& *\left(e^{1} \wedge e^{4}\right)=e^{2} \wedge e^{3} \\
& *\left(e^{2} \wedge e^{3}\right)=e^{1} \wedge e^{4} \\
& *\left(e^{2} \wedge e^{4}\right)=e^{3} \wedge e^{1} \\
& *\left(e^{3} \wedge e^{4}\right)=e^{1} \wedge e^{2}
\end{aligned}
$$

esse quadro pode ser encontrado em [39]. Isso implica que $*$ é uma involução, i.e. $*^{2}=I d$. Vamos denotar por $(\bar{r} \bar{s})$ o dual de $(r s)$, isso quer dizer que, $(r s \bar{r} \bar{s})=\sigma(1234)$ para alguma permutação $\sigma$ no conjunto $\{1,2,3,4\}$ (cf. p. 466 em [26]). Como o operador de Hodge é auto-adjunto, i.e

$$
\left\langle\left\langle *\left(e^{i} \wedge e^{j}\right), e^{k} \wedge e^{l}\right\rangle\right\rangle=\left\langle\left\langle e^{i} \wedge e^{j}, *\left(e^{k} \wedge e^{l}\right)\right\rangle\right\rangle
$$

onde $\langle\langle\cdot, \cdot\rangle\rangle$ é o produto interno para fibrado das 2-formas, $\Lambda^{2}$ (bivetores), os únicos autovalores de $*$ são \pm 1 (cf. [39]). Por isso o fibrado das 2-formas, em uma 4-variedade Riemanniana orientada, pode ser decomposto em soma direta $\Lambda^{2}=\Lambda_{+}^{2} \oplus \Lambda_{-}^{2}$. Isso nos 
permite concluir que o tensor de Weyl $W$ é um endomorfismo de $\Lambda^{2}=\Lambda^{+} \oplus \Lambda^{-}$tal que

$$
W=W^{+} \oplus W^{-}
$$

Lembramos que $\operatorname{dim}_{\mathbb{R}}\left(\Lambda^{2}\right)=6$ e $\operatorname{dim}_{\mathbb{R}}\left(\Lambda^{ \pm}\right)=3$. Além disso, as bases para $\Lambda^{+}$e $\Lambda^{-}$são (cf. [26]):

$$
\Lambda^{+}=\operatorname{span}\left\{\frac{e^{1} \wedge e^{2}+e^{3} \wedge e^{4}}{\sqrt{2}}, \frac{e^{1} \wedge e^{3}+e^{4} \wedge e^{2}}{\sqrt{2}}, \frac{e^{3} \wedge e^{2}+e^{4} \wedge e^{1}}{\sqrt{2}}\right\}
$$

e

$$
\Lambda^{-}=\operatorname{span}\left\{\frac{e^{1} \wedge e^{2}-e^{3} \wedge e^{4}}{\sqrt{2}}, \frac{e^{1} \wedge e^{3}-e^{4} \wedge e^{2}}{\sqrt{2}}, \frac{e^{3} \wedge e^{2}-e^{4} \wedge e^{1}}{\sqrt{2}}\right\} .
$$

Por isso, $\Lambda^{+}$e $\Lambda^{-}$tem uma orientação natural tal que as bases (1.22) e (1.23) são positivamente orientadas. Além do mais, se $\mathcal{R}$ é o operador curvatura em $M^{4}$ temos a matriz

$$
\mathcal{R}=\left(\begin{array}{c|c}
W^{+}+\frac{R}{12} I d & \text { Ric } \\
\hline \text { Ric }^{\star} & W^{-}+\frac{R}{12} I d
\end{array}\right)
$$

onde Ric $: \Lambda^{-} \rightarrow \Lambda^{+}$é o operador de Ricci sem traço em $M^{4}$ definido em (1.10).

Como o tensor de Weyl é livre de traço (i.e, $\operatorname{tr} W=0$ ) em qualquer par de índices temos que

$$
W_{p q r s}^{+}=\frac{1}{2}\left(W_{p q r s}+W_{p q \bar{r} \bar{s}}\right)
$$

Em particular, temos que

$$
W_{1234}^{+}=\frac{1}{2}\left(W_{1234}+W_{1212}\right) .
$$

Para maiores detalhes confira [26] e [56].

Sendo $W^{+}$um tensor do tipo $(0,4)$, podemos dizer que $W^{+}$é harmônico se $\delta W^{+}=0$, onde $\delta$ é o divergente. O divergente de um $(0,4)$-tensor $T$ é definido por

$$
\delta T\left(X_{1}, X_{2}, X_{3}\right)=\operatorname{traço~}_{g}\left\{(Y, Z) \mapsto \nabla_{Y} T\left(Z, X_{1}, X_{2}, X_{3}\right)\right\}
$$

onde $g$ é a métrica de $M^{4}$. Além disso, é bom enfatizar que variedades de Einstein 4-dimensional possuem tensor $W^{+}$harmônico (cf. 16.65 in [9], ver também o Lema 
6.14 em [26]). Portanto, é natural nos perguntarmos que implicações geométricas tem a harmonicidade do tensor $W^{+}$.

\subsection{Métrica CPE (critical point equation)}

Definição 1.39. Uma métrica $C P E$ é uma tripla $\left(M^{n}, g, f\right)$, onde $\left(M^{n}, g\right)$ é uma variedade Riemanniana orientada compacta com dimensão $n \geq 3$, volume unitário e curvatura escalar constante com uma função diferenciável $f$ satisfazendo

$$
(1+f) \stackrel{\circ}{\operatorname{Ric}}=\nabla^{2} f+\frac{R f}{n(n-1)} g,
$$

onde $\stackrel{\circ}{\text { Ric }}$, $R$ e $\nabla^{2}=$ Hess são, respectivamente, o tensor de Ricci sem traço $(\stackrel{\circ}{\text { Ric }}=$ Ric $-\frac{R}{n} g$ ), a curvatura escalar e o Hessiano da métrica $g$ em $M^{n}$.

É claro que a métrica de Einstein é canônica quando $f=0$. Além disso, a única solução não-constante conhecida é a esfera com função altura $f$. Foi conjecturado que a métrica CPE é uma métrica de Einstein. Para maiores detalhes, confira [9] p. 128.

Note que, se contrairmos a equação (1.26), obtemos

$$
\Delta f+\frac{R}{(n-1)} f=0
$$

Portanto $f$ é uma autofunção do laplaciano e então, pela teoria espectral, $R$ é positivo.

Em particular, se a métrica CPE for Einstein, então pela equação fundamental (1.26) teremos para quaisquer campos vetoriais $X$ e $Y$

$$
\nabla^{2} f(X, Y)=-\frac{R f}{n(n-1)} g(X, Y)
$$

nos dando que $\nabla f$ é um campo vetorial conforme. Nesse caso, podemos usar o teorema de Obata [48] para concluir que $M^{n}$ é isométrica a esfera $\mathbb{S}^{n}(\rho), \operatorname{com} \rho=\sqrt{\frac{n(n-1)}{R}}$.

Teorema 1.40. [48] Uma variedade Riemanniana completa de dimensão $n \geq 2$ admite uma função não-constante $f$ com $\nabla^{2} f(X, Y)=-c^{2} f g(X, Y)$ para quaisquer campos vetoriais $X$ e $Y$ se, e somente se, a variedade é isométrica a esfera $\mathbb{S}^{n}(c)$ de raio $\frac{1}{c}$, com $c>0$ constante.

A conjectura proposta em [9] em meados dos anos 1980 pode ser reescrita em termos CPE (cf. [18, 35, 36, 52]). Para ser mais preciso, esses autores propuseram o seguinte: 
Conjectura 1.41. [9] A métrica CPE é sempre Einstein. 


\section{Capítulo 2}

\section{Solitons gradientes de Yamabe conformes a um espaço pseudo-Euclidiano}

Consideremos solitons gradientes de Yamabe (ver Seção 1.4), conformes a espaços pseudo-Euclidianos que são invariantes pela ação de um grupo de translação ( $n$ 1)-dimensional (ver Seções 1.1 e 1.3). Precisamente, seja $\left(\mathbb{R}^{n}, g\right)$ um espaço pseudoeuclidiano com métrica $g$ e coordenadas $\left(x_{1}, \cdots, x_{n}\right)$, com $g_{i j}=\delta_{i j} \varepsilon_{i}, 1 \leq i, j \leq n$, onde $\delta_{i j}$ é o delta de Kronecker, $\varepsilon_{i}= \pm 1$, com pelo menos um $\varepsilon_{i}=1$. Seja $\xi=\sum_{i} \alpha_{i} x_{i}$, $\alpha_{i} \in \mathbb{R}$, um invariante básico para um grupo de translação $(n-1)$-dimensional. Nós queremos obter funções diferenciáveis $\varphi(\xi)$ e $f(\xi)$, tais que a métrica $\bar{g}=\frac{g}{\varphi^{2}}$ satisfaça a equação

$$
\operatorname{Hess}_{\bar{g}}(f)=\left(R_{\bar{g}}-\lambda\right) \bar{g}
$$

onde $R_{\bar{g}}$ é a curvatura escalar da métrica $\bar{g}$.

Primeiramente, nós obtemos condições necessárias e suficientes sobre $f(\xi)$ e $\varphi(\xi)$ para a existência da métrica $\bar{g}$ (não necessariamente completa). Veremos que essas condições são diferentes dependendo da direção do vetor $\alpha=\sum_{i=1}^{n} \alpha_{i} \frac{\partial}{\partial x_{i}}$ ser tipo luz ou não.

Em seguida, vamos construir um exemplo explícito de soliton gradiente de Yamabe, conforme a um espaço pseudo-Euclidiano que é invariante pela a ação de um grupo de translação $(n-1)$-dimensional que é completo tipo espaço (ou tempo) e tipo luz.

Nos cálculos, nós denotaremos por $\varphi_{x_{i} x_{j}}$ e $f_{x_{i} x_{j}}$ as derivadas de segunda ordem de $\varphi$ e $f$, com respeito a $x_{i} x_{j}$. Vamos prosseguir enunciando os resultados principais do 
capítulo.

Nosso primeiro teorema mostra o sistema de equações que as funções $\varphi$ e $f$ satisfazem quando o soliton gradiente de Yamabe é conforme a um espaço pseudo-Euclidiano.

Teorema 2.1. Seja $\left(\mathbb{R}^{n}, g\right)$ um espaço pseudo-Euclidiano com $n \geq 3$, coordenadas $x=$ $\left(x_{1}, \ldots, x_{n}\right)$ e $g_{i j}=\delta_{i j} \varepsilon_{i}$ e seja $f: \mathbb{R}^{n} \rightarrow \mathbb{R}$ uma função diferenciável. Então existe uma métrica $\bar{g}=\frac{g}{\varphi^{2}}$ tal que $\left(\mathbb{R}^{n}, \bar{g}\right)$ é um soliton gradiente de Yamabe com função potencial $f$ se, e somente se, as funções $\varphi$ e $f$ satisfazem

$$
f_{x_{i} x_{j}}+\frac{\varphi_{x_{i}} f_{x_{j}}}{\varphi}+\frac{\varphi_{x_{j}} f_{x_{i}}}{\varphi}=0, \quad i \neq j
$$

e para cada $i$

$$
f_{x_{i} x_{i}}+2 \frac{\varphi_{x_{i}} f_{x_{i}}}{\varphi}-\varepsilon_{i} \sum_{k=1}^{n} \varepsilon_{k} \frac{\varphi_{x_{k}}}{\varphi} f_{x_{k}}=\frac{\varepsilon_{i}}{\varphi^{2}}\left[(n-1)\left(2 \varphi \Delta_{g} \varphi-n\left|\nabla_{g} \varphi\right|^{2}\right)-\lambda\right] .
$$

Nós queremos encontrar soluções para os sistemas (2.1), (2.2) da forma $\varphi(\xi)$ e $f(\xi)$, onde $\xi=\sum_{k=1}^{n} \alpha_{k} x_{k}, \alpha_{k} \in \mathbb{R}$. Sempre que $\sum_{k=1}^{n} \varepsilon_{k} \alpha_{k}^{2} \neq 0$, sem perda de generalidade, podemos considerar $\sum_{k=1}^{n} \varepsilon_{k} \alpha_{k}^{2}= \pm 1$. O teorema seguinte mostra o sistema de equações diferenciais ordinárias que tais soluções devem satisfazer.

A seguir, consideram o soliton do Teorema 2.1, mostraremos o sistema de equações diferenciáveis ordinárias que as funções conforme e potencial satisfazem quando o soliton é invariante pela ação de um grupo de translação.

Teorema 2.2. Seja $\left(\mathbb{R}^{n}, g\right)$ um espaço pseudo-Euclidiano com $n \geq 3$, coordenadas $x=$ $\left(x_{1}, \ldots, x_{n}\right)$ e $g_{i j}=\delta_{i j} \varepsilon_{i}$. Considere as funções diferenciáveis $\varphi(\xi)$ e $f(\xi)$, onde $\xi=$ $\sum_{k=1}^{n} \alpha_{k} x_{k}, \alpha_{k} \in \mathbb{R} e \sum_{k=1}^{n} \varepsilon_{k} \alpha_{k}^{2}=\varepsilon_{k_{0}}$ ou $\sum_{k=1}^{n} \varepsilon_{k} \alpha_{k}^{2}=0$. Então $\bar{g}=\frac{g}{\varphi^{2}}$ é um soliton gradiente de Yamabe com função potencial $f$ se, e somente se, $\varphi$ e $f$ satisfazem

$$
\left\{\begin{array}{l}
f^{\prime \prime}+2 \frac{\varphi^{\prime} f^{\prime}}{\varphi}=0 ; \\
-\varphi^{\prime} \varphi f^{\prime}=(n-1)\left[2 \varphi \varphi^{\prime \prime}-n\left(\varphi^{\prime}\right)^{2}\right]-\varepsilon_{k_{0}} \lambda,
\end{array} \quad \text { se } \sum_{k=1}^{n} \varepsilon_{k} \alpha_{k}^{2}=\varepsilon_{k_{0}}\right.
$$




$$
\begin{cases}f^{\prime \prime}+2 \frac{\varphi^{\prime} f^{\prime}}{\varphi}=0 ; & \text { se } \sum_{k=1}^{n} \varepsilon_{k} \alpha_{k}^{2}=0 \\ \lambda=0 & \end{cases}
$$

Os próximos resultados buscam as soluções de (2.2) e (2.3), quando $\lambda=0$, isto é, no caso estável.

Teorema 2.3. Seja $\left(\mathbb{R}^{n}, g\right)$ um espaço pseudo-Euclidiano com $n \geq 3$, coordenadas $x=$ $\left(x_{1}, \ldots, x_{n}\right)$ e $g_{i j}=\delta_{i j} \varepsilon_{i}$. Seja $\bar{g}=\frac{g}{\varphi^{2}}$, um soliton gradiente de Yamabe estável, com função potencial $f$. Então $\varphi$ e $f$ são invariantes sob a ação de um grupo de translação de dimensão $(n-1)$ cujo invariante básico é $\xi=\sum_{k=1}^{n} \alpha_{k} x_{k}$, onde $\alpha=\sum_{k=1}^{n} \alpha_{k} \frac{\partial}{\partial x_{k}}$ é um vetor não-nulo com $\sum_{k=1}^{n} \varepsilon_{k} \alpha_{k}^{2}=\varepsilon_{k_{0}}$ se, e somente se,

$$
\int \frac{\varphi}{\gamma-\beta \varphi^{\frac{n}{2}+1}} d \varphi=\frac{\xi+\nu}{(n-1)(n+2)}
$$

$e$

$$
f^{\prime}(\xi)=\frac{\gamma}{\varphi(\xi)^{2}}, \quad \gamma \in \mathbb{R}
$$

onde $\nu, \beta \in \mathbb{R}, \gamma^{2}+\beta^{2} \neq 0$. Em particular, quando $\gamma=0$, então $f$ é constante $e\left(\mathbb{R}^{n}, \bar{g}\right)$ é um soliton gradiente de Yamabe estável trivial. Se g é a métrica Euclidiana então $\bar{g}$ não é completo.

Teorema 2.4. Seja $\left(R^{n}, g\right)$ um espaço pseudo-Euclidiano com $n \geq 3$, coordenadas $x=$ $\left(x_{1}, \ldots, x_{n}\right)$ e $g_{i j}=\delta_{i j} \varepsilon_{i}$. Seja $\bar{g}=g / \varphi^{2}$, um soliton gradiente de Yamabe estável com função potencial $f$. Então $\varphi$ e $f$ são invariantes pela ação do grupo de translaçãa de dimensão $(n-1)$, cujo invariante básico é $\xi=\sum_{k=1}^{n} \alpha_{k} x_{k}$ onde $\alpha=\sum_{k=1}^{n} \alpha_{k} \frac{\partial}{\partial x_{k}}$ é um vetor 
tipo luz (lightlike), isto é, $\left(\sum_{k=1}^{n} \varepsilon_{k} \alpha_{k}^{2}=0\right)$ se, e somente se, $\varphi$ é uma função diferenciável qualquer não-nula e

$$
f(\xi)=\gamma \int \frac{1}{\varphi^{2}(\xi)} d \xi, \quad \gamma \in \mathbb{R}
$$

Antes de provar os resultados acima enunciados, vamos ilustrar os Teoremas 2.3 e 2.4 com alguns exemplos.

Exemplo 2.5. Se considerarmos $\gamma \neq 0$ e $\beta=0$ no Teorema 2.3, então temos que

$$
\varphi^{2}(\xi)=\frac{2 \gamma(\xi+\nu)}{(n-1)(n+2)} \quad \text { e } \quad f(\xi)=\frac{1}{2}(n-1)(n+2) \ln |\xi+\nu|
$$

onde $\gamma(\xi+\nu)>0$. Portanto, $\bar{g}=\frac{g}{\varphi^{2}}$ é um soliton gradiente de Yamabe estável no semi-espaço de $\mathbb{R}^{n}$, com função potencial $f$.

Exemplo 2.6. No Teorema 2.3, considere $\left(\mathbb{R}^{6}, g\right)$ um espaço pseudo-Euclidiano de dimensão $n=6$ e $\xi=\sum_{k=1}^{6} \alpha_{k} x_{k}$ tal que $\sum_{k=1}^{6} \varepsilon_{k} \alpha_{k}^{2}=\varepsilon_{k_{0}}$. Se $\gamma \beta>0$, então podemos resolver explicitamente as equações (2.5) e (2.4). Na verdade, obtemos

$$
\varphi^{2}(\xi)=\sqrt{\frac{\gamma}{\beta}} \operatorname{coth}\left(\frac{(\xi+\nu) \sqrt{\gamma \beta}}{20}\right) \quad \text { e } \quad f(\xi)=20 \ln \left[\operatorname{coth}\left(\frac{(\xi+\nu) \sqrt{\gamma \beta}}{20}\right)\right],
$$

onde $\xi+\nu>0$. Segue que $\bar{g}=\frac{g}{\varphi^{2}}$ é um soliton gradiente de Yamabe estável no semiespaço de $\mathbb{R}^{6}$, com função potencial $f$.

Exemplo 2.7. Sob as mesmas condições do exemplo acima, se as constantes $\gamma$ e $\beta$ satisfazem $\gamma \beta<0$, então podemos resolver explicitamente as equações (2.5) e (2.4). De fato, encontraremos

$$
\varphi^{2}(\xi)=\sqrt{\left|\frac{\gamma}{\beta}\right|} \tan \left(\operatorname{sgn} \gamma \frac{(\xi+\nu) \sqrt{|\gamma \beta|}}{20}\right) \quad \text { e } \quad f(\xi)=20 \ln \left[\sin \left(\operatorname{sgn} \gamma \frac{(\xi+\nu) \sqrt{|\gamma \beta|}}{20}\right)\right]
$$

onde $\xi+\nu>0$. Segue que $\bar{g}=\frac{g}{\varphi^{2}}$ é um soliton gradiente de Yamabe estável no semiespaço de $\mathbb{R}^{6}$, com função potencial $f$.

Exemplo 2.8. Agora, um exemplo para o Teorema 2.4. Seja $\left(\mathbb{R}^{n}, g\right)$ um espaço Lorentziano com coordenadas $\left(x_{1}, \ldots, x_{n}\right)$ e assinatura: $\varepsilon_{1}=-1, \varepsilon_{k}=1$, para $k \geq 2$. Considere 
$\xi=x_{1}+x_{2}$ e escolha $\varphi(\xi)=\frac{1}{1+\xi^{2}}$. Então,

$$
\bar{g}=\left(1+\xi^{2}\right)^{2} g \quad \text { e } \quad f(\xi)=\gamma \xi\left(1+\frac{2}{3} \xi^{2}+\frac{1}{5} \xi^{4}\right)
$$

onde $\gamma \in \mathbb{R} \backslash\{0\}$. Segue que $\left(\mathbb{R}^{n}, \bar{g}\right)$ é um soliton gradiente de Yamabe estável, com função potencial $f$. Mostraremos no última seção desse capítulo que este soliton gradiente de Yamabe é geodesicamente completo. 


\subsection{Demonstração dos resultados principais}

Demonstração do Teorema 2.1: Sabemos que se $\bar{g}=\frac{1}{\varphi^{2}} g$, então

$$
\operatorname{Ric}_{\bar{g}}=\frac{1}{\varphi^{2}}\left\{(n-2) \varphi \operatorname{Hess}_{g}(\varphi)+\left[\varphi \Delta_{g} \varphi-(n-1)\left|\nabla_{g} \varphi\right|^{2}\right] g\right\}
$$

Portanto, por (2.6) a curvatura escalar da métrica $\bar{g}$ é dada por

$$
R_{\bar{g}}=\sum_{k=1}^{n} \varepsilon_{k} \varphi^{2}\left(\operatorname{Ric}_{\bar{g}}\right)_{k k}=(n-1)\left(2 \varphi \Delta_{g} \varphi-n\left|\nabla_{g} \varphi\right|^{2}\right)
$$

Então, a equação

$$
\operatorname{Hess}_{\bar{g}}(f)=\left(R_{\bar{g}}-\lambda\right) \bar{g}
$$

é equivalente a

$$
\operatorname{Hess}_{\bar{g}}(f)=\left((n-1)\left(2 \varphi \Delta_{g} \varphi-n\left|\nabla_{g} \varphi\right|^{2}\right)-\lambda\right) \bar{g}
$$

Lembrando que

$$
\operatorname{Hess}_{\bar{g}}(f)_{i j}=f_{x_{i} x_{j}}-\sum_{k} \bar{\Gamma}_{i j}^{k} f_{x_{k}}
$$

onde $\bar{\Gamma}_{i j}^{k}$ são os símbolos de christoffel da métrica $\bar{g}$. Para $i, j, k$ distintos, já que $\bar{g}=\frac{\varepsilon_{i} \delta_{i j}}{\varphi^{2}}$ e $(\bar{g})^{-1}=\varphi^{2} \varepsilon_{i} \delta_{i j}$, nós temos por (1.2)

$$
\begin{aligned}
\Gamma_{i j}^{k} & =\frac{1}{2} \sum_{m} g^{m k}\left[\frac{\partial}{\partial x_{i}} g_{j m}+\frac{\partial}{\partial x_{j}} g_{i m}-\frac{\partial}{\partial x_{m}} g_{i j}\right] \\
& =\frac{1}{2} g^{k k}\left[\frac{\partial}{\partial x_{i}} g_{j k}+\frac{\partial}{\partial x_{j}} g_{i k}-\frac{\partial}{\partial x_{k}} g_{i j}\right]=0, \\
\Gamma_{i j}^{i}= & \frac{1}{2} \sum_{m} g^{m i}\left[\frac{\partial}{\partial x_{i}} g_{j m}+\frac{\partial}{\partial x_{j}} g_{i m}-\frac{\partial}{\partial x_{m}} g_{i j}\right] \\
= & \frac{1}{2} g^{i i}\left[\frac{\partial}{\partial x_{i}} g_{j i}+\frac{\partial}{\partial x_{j}} g_{i i}-\frac{\partial}{\partial x_{i}} g_{i j}\right] \\
= & \frac{1}{2} \varphi^{2} \varepsilon_{i}\left[\frac{\partial}{\partial x_{j}} g_{i i}\right] \\
= & \frac{1}{2} \varphi^{2} \varepsilon_{i}\left[\frac{\partial}{\partial x_{j}} \varepsilon_{i} \varphi^{-2}\right]=\frac{1}{2} \varphi^{2} \varepsilon_{i}\left[-2 \varepsilon_{i} \frac{\varphi_{x_{j}}}{\varphi^{3}}\right]=-\frac{\varphi_{x_{j}}}{\varphi},
\end{aligned}
$$




$$
\begin{aligned}
& \Gamma_{i i}^{k}=\frac{1}{2} \sum_{m} g^{m k}\left[\frac{\partial}{\partial x_{i}} g_{i m}+\frac{\partial}{\partial x_{i}} g_{i m}-\frac{\partial}{\partial x_{m}} g_{i i}\right] \\
&=\frac{1}{2} g^{k k}\left[\frac{\partial}{\partial x_{i}} g_{i k}+\frac{\partial}{\partial x_{i}} g_{i k}-\frac{\partial}{\partial x_{k}} g_{i i}\right] \\
&=\frac{1}{2} \varphi^{2} \varepsilon_{k}\left[-\frac{\partial}{\partial x_{k}} \varepsilon_{i} \varphi^{-2}\right]=\frac{1}{2} \varphi^{2} \varepsilon_{k}\left[2 \varepsilon_{i} \frac{\varphi_{x_{k}}}{\varphi^{3}}\right]=\varepsilon_{i} \varepsilon_{k} \frac{\varphi_{x_{k}}}{\varphi}, \\
& \Gamma_{i i}^{i}=\frac{1}{2} \sum_{m} g^{m i}\left[\frac{\partial}{\partial x_{i}} g_{i m}+\frac{\partial}{\partial x_{i}} g_{i m}-\frac{\partial}{\partial x_{m}} g_{i i}\right] \\
&=\frac{1}{2} g^{i i}\left[\frac{\partial}{\partial x_{i}} g_{i i}+\frac{\partial}{\partial x_{i}} g_{i i}-\frac{\partial}{\partial x_{i}} g_{i i}\right] \\
&=\frac{1}{2} g^{i i}\left[\frac{\partial}{\partial x_{i}} g_{i i}\right]=\frac{1}{2} \varepsilon_{i} \varphi^{2}\left[\frac{\partial}{\partial x_{i}} \varepsilon_{i} \varphi^{-2}\right]=\frac{1}{2} \varepsilon_{i} \varphi^{2}\left[-2 \varepsilon_{i} \frac{\varphi_{x_{i}}}{\varphi^{3}}\right]=-\frac{\varphi_{x_{i}}}{\varphi} .
\end{aligned}
$$

Logo,

$$
\operatorname{Hess}_{\bar{g}}(f)_{i j}=f_{x_{i} x_{j}}+\frac{\varphi_{x_{i}} f_{x_{j}}}{\varphi}+\frac{\varphi_{x_{j}} f_{x_{i}}}{\varphi} ; \quad i \neq j .
$$

Substituindo essa expressão em (2.8), se $i \neq j$ temos (2.1).

Analogamente, quando $i=j$, obtemos

$$
\operatorname{Hess}_{\bar{g}}(f)_{i i}=f_{x_{i} x_{i}}+2 \frac{\varphi_{x_{i}} f_{x_{i}}}{\varphi}-\varepsilon_{i} \sum_{k} \varepsilon_{k} \frac{\varphi_{x_{k}}}{\varphi} f_{x_{k}}
$$

E substituindo essa expressão em (2.8), vamos ter (2.2). Um cálculo direto nos mostra a implicação contrária. Isso conclui a demonstração do Teorema 2.1.

Demonstração do Teorema 2.2: Seja $\bar{g}=\frac{1}{\varphi^{2}} g$ a métrica conforme de $g$. Assumindo que $\varphi(\xi)$ e $f(\xi)$ são funções de $\xi$, onde $\xi=\sum_{k=1}^{n} \alpha_{k} x_{k}, \alpha_{k} \in \mathbb{R}$ e $\sum_{k=1}^{n} \varepsilon_{k} \alpha_{k}^{2}=\varepsilon_{k_{0}}$ ou $\sum_{k=1}^{n} \varepsilon_{k} \alpha_{k}^{2}=0$, temos que

$$
\varphi_{x_{i}}=\varphi^{\prime} \alpha_{i} ; \quad \varphi_{x_{i} x_{j}}=\varphi^{\prime \prime} \alpha_{i} \alpha_{j} \quad \text { e } \quad f_{x_{i}}=f^{\prime} \alpha_{i} ; \quad f_{x_{i} x_{j}}=f^{\prime \prime} \alpha_{i} \alpha_{j} .
$$

Substituindo essas expressões em (2.1), nós temos

$$
\left(f^{\prime \prime}+2 \frac{\varphi^{\prime} f^{\prime}}{\varphi}\right) \alpha_{i} \alpha_{j}=0 ; \quad \forall i \neq j
$$


Analogamente, considerando a equação (2.2), temos para todo $i$,

$$
\begin{aligned}
\left(f^{\prime \prime}+2 \frac{\varphi^{\prime} f^{\prime}}{\varphi}\right) \alpha_{i}^{2}-\varepsilon_{i} \frac{\varphi^{\prime} f^{\prime}}{\varphi} \sum_{k=1}^{n} \varepsilon_{k} \alpha_{k}^{2} & =\frac{\varepsilon_{i}}{\varphi^{2}}\left\{( n - 1 ) \sum _ { k = 1 } ^ { n } \varepsilon _ { k } \alpha _ { k } ^ { 2 } \left[2 \varphi \varphi^{\prime \prime}\right.\right. \\
& \left.\left.-n\left(\varphi^{\prime}\right)^{2}\right]-\lambda\right\}
\end{aligned}
$$

Se existe $i$ e $j, i \neq j$, tais que $\alpha_{i} \alpha_{j} \neq 0$, então a equação (2.13) nos dá que

$$
f^{\prime \prime}+2 \frac{\varphi^{\prime} f^{\prime}}{\varphi}=0
$$

Segue de (2.15) que a equação (2.14) se reduz a

$$
-\frac{\varphi^{\prime} f^{\prime}}{\varphi} \sum_{k=1}^{n} \varepsilon_{k} \alpha_{k}^{2}=\frac{1}{\varphi^{2}} \sum_{k=1}^{n} \varepsilon_{k} \alpha_{k}^{2}(n-1)\left(2 \varphi \varphi^{\prime \prime}-n\left(\varphi^{\prime}\right)^{2}\right)-\frac{\lambda}{\varphi^{2}}
$$

Portanto, se $\sum_{k=1}^{n} \varepsilon_{k} \alpha_{k}^{2}=\varepsilon_{k_{0}}$, nós obtemos a segunda equação do sistema (2.2). Se $\sum_{k=1}^{n} \varepsilon_{k} \alpha_{k}^{2}=0$, então existe $i$ e $j, i \neq j$, tais que $\alpha_{i} \alpha_{j} \neq 0$, segue de (2.16) que $\lambda=0$.

Nós precisamos considerar o caso em que $\alpha_{k_{0}}=1$ e $\alpha_{k}=0, \forall k \neq k_{0}$. Nesse caso, (2.13) é satisfeita trivialmente e nós obtemos, por (2.14), que

$$
\left\{\begin{array}{l}
-\varphi \varphi^{\prime} f^{\prime}=(n-1)\left[2 \varphi \varphi^{\prime \prime}-n\left(\varphi^{\prime}\right)^{2}\right]-\varepsilon_{k_{0}} \lambda ; \quad i \neq k_{0} \\
f^{\prime \prime}+\frac{\varphi^{\prime} f^{\prime}}{\varphi}=\frac{1}{\varphi^{2}}\left\{(n-1)\left[2 \varphi \varphi^{\prime \prime}-n\left(\varphi^{\prime}\right)^{2}\right]-\varepsilon_{k_{0}} \lambda\right\} ; \quad i=k_{0} .
\end{array}\right.
$$

Esse sistema de equações é equivalente a (2.2).

Demonstração do Teorema 2.3: Para demonstrar o Teorema 2.3, nós vamos considerar as funções diferenciáveis $\varphi(\xi)$ e $f(\xi)$, onde $\xi=\sum_{k=1}^{n} \alpha_{k} x_{k}, \alpha_{k} \in \mathbb{R}, \sum_{k=1}^{n} \varepsilon_{k} \alpha_{k}^{2}=\varepsilon_{k_{0}}$. Segue do Teorema 2.2 que $\bar{g}=\frac{1}{\varphi^{2}} g$ é um soliton gradiente de Yamabe estável com função potencial $f$ se, e somente se, $\varphi$ e $f$ satisfazem $(2.2) \operatorname{com} \lambda=0$. 
A primeira equação do sistema (2.2) implica em

$$
f^{\prime}=\gamma \varphi^{-2}, \forall \gamma \in \mathbb{R}
$$

Substituindo (2.17) na segunda equação do sistema, obtemos

$$
\varphi \varphi^{\prime \prime}-\frac{n}{2}\left(\varphi^{\prime}\right)^{2}+\frac{\gamma}{2(n-1)} \frac{\varphi^{\prime}}{\varphi}=0 .
$$

Considere

$$
\varphi(\xi)^{1-\frac{n}{2}}=\omega(\xi)
$$

teremos então

$$
\omega^{\prime}=\left(1-\frac{n}{2}\right) \varphi^{-\frac{n}{2}} \varphi^{\prime} \quad \text { and } \quad \omega^{\prime \prime}=\left(1-\frac{n}{2}\right) \varphi^{-\frac{n}{2}-1}\left(\varphi \varphi^{\prime \prime}-\frac{n}{2}\left(\varphi^{\prime}\right)^{2}\right)
$$

Então, a equação (2.18) é equivalente a

$$
\omega^{\prime \prime}+\frac{\gamma}{2(n-1)} \omega^{\prime} \omega^{\frac{4}{(n-2)}}=0
$$

Integrando (2.19), obtemos

$$
\omega^{\prime}+\frac{\gamma(n-2)}{2(n-1)(n+2)} \omega^{\frac{(n+2)}{(n-2)}}=\sigma ; \quad \sigma \in \mathbb{R} .
$$

Isso implica que

$$
-\int \frac{1}{\frac{\gamma(n-2)}{2(n-1)(n+2)} \omega^{\frac{(n+2)}{(n-2)}}-\sigma} d \omega=\xi+\nu .
$$

Segue de (2.20) que

$$
\frac{(n-2)}{2} \int \frac{d \varphi}{\frac{\gamma(n-2)}{2(n-1)(n+2)} \varphi^{-1}-\sigma \varphi^{\frac{n}{2}}}=\xi+\nu
$$

Já que $\sigma$ é uma constante arbitrária, vamos ter que

$$
(n-1)(n+2) \int \frac{\varphi d \varphi}{\gamma-\beta \varphi^{\frac{n}{2}+1}}=\xi+\nu, \quad \gamma^{2}+\beta^{2} \neq 0,
$$

onde $\beta \in \mathbb{R}$, isto é, teremos (2.4). 
Demonstração do Teorema 2.4: Segue imediatamente como consequência de (2.3).

\subsection{Exemplo de soliton gradiente de Yamabe semi- Riemanniano estável completo}

Nessa seção, nós demonstraremos que o Exemplo 2.8 é soliton gradiente de Yamabe completo conforme a um espaço Lorentziano. Exemplos de variedades pseudo-Riemannianas são encontrados na literatura. Mas não existem muitos resultados sobre a completude de espaços Pseudo-Riemannianos. Kundt [40] construiu um exemplo de variedade que é completa tipo tempo e luz, mas que não é completa tipo espaço. Em seguida Geroch [28] nos deu um exemplo de espaço-tempo conforme a um 2-espaço de Minkowski e assim globalmente hiperbólico que é incompleto tipo tempo mas completo tipo espaço e luz. Também Geroch observou que fazendo algumas modificações no exemplo de Kundt teriamos espaço-tempos que são (1) incompletos em dois tipos mas completo no terceiro, (2) espaço incompleto mas completo tipo luz e tempo, e (3) incompleto tipo tempo mas completo no tipo espaço e luz. Então Beem [6] construiu um exemplo de espaço-tempo globalmente hiperbólico que é incompleto tipo luzmas completo tipo espaço e tempo. Na verdade, podemos construir um exemplo de espaço-tempo que é incompleto tipo espaço mas completo nos outros dois tipos em produtos torcidos ([11], Teorema 3.40). Para qualquer métrica conforme de uma variedade semi-Riemanniana indefinida existe um resultado de completude tipo luz ([11], Teorema 2.2).

Um cálculo direto, utilizando a fórmula de Koszul (1.1) fornece a relação entre a conexão de Levi-Civita $\nabla, \bar{\nabla}$ respectivamente correspondentes a $g$ e $\bar{g}=\frac{g}{\varphi^{2}}$ :

$$
\bar{\nabla}_{X} Y=\nabla_{X} Y-\frac{1}{\varphi}\left[(X \varphi) Y+(Y \varphi) X-g(X, Y) \nabla_{g} \varphi\right]
$$

onde $Z \varphi=g\left(\nabla_{g} \varphi, Z\right)$ e $X, Y, Z$ campos vetoriais em $\mathbb{R}^{n}$. Conseqüentemente, obtemos a relação entre as derivadas covariantes $\frac{D}{d t}, \frac{\bar{D}}{d t}$ e teremos a seguinte igualdade para uma 
curva qualquer $\phi: I \subset \mathbb{R} \rightarrow\left(\mathbb{R}^{n}, \bar{g}\right)$ (ver, [11] pg:10):

$$
\frac{\bar{D}}{d t} \phi^{\prime}=\frac{D}{d t} \phi^{\prime}-\frac{1}{\varphi}\left[2 d \varphi\left(\phi^{\prime}(t)\right) \cdot \phi^{\prime}-g\left(\phi^{\prime}, \phi^{\prime}\right) \nabla_{g} \varphi\right]
$$

Prova da completude do Exemplo 2.8: Seja $\left(\mathbb{R}^{n}, g\right)$ um espaço pseudo-Euclidiano com coordenadas $\left(x_{1}, \ldots, x_{n}\right)$ e assinatura $\varepsilon_{1}=-1, \varepsilon_{k}=1$, para $k \geq 2$. Considere, assim como no Exemplo 2.8,

$$
\xi=x_{1}+x_{2}, \quad \bar{g}=\left(1+\xi^{2}\right)^{2} g \quad \text { e } \quad f=\gamma \xi\left(1+\frac{2}{3} \xi^{2}+\frac{1}{5} \xi^{4}\right)
$$

onde $\gamma \in \mathbb{R} \backslash\{0\}$. Então, afirmamos que $(\mathbb{R}, \bar{g}, f)$ é um soliton gradiente de Yamabe estável completo com função potencial $f$.

De fato, considerando $\xi=\sum_{k=1}^{n} \alpha_{k} x_{k}$, onde $\alpha_{1}=\alpha_{2}=1$ e $\alpha_{\ell}=0$ para $\ell \geq 3$, temos que $\sum_{k=1}^{n} \varepsilon_{k} \alpha_{k}^{2}=0$. Segue do Teorema 2.4 que, considerando

$$
\varphi(\xi)=\frac{1}{1+\xi^{2}}
$$

$f$ é a função potencial do soliton gradiente de Yamabe estável $(\mathbb{R}, \bar{g}, f)$.

Antes de provar que ele é completo, observe que um cálculo simples nos mostra que a curvatura seccional é dada por

$$
\begin{gathered}
K\left(\frac{\partial}{\partial x_{1}}, \frac{\partial}{\partial x_{\ell}}\right)=-K\left(\frac{\partial}{\partial x_{2}}, \frac{\partial}{\partial x_{\ell}}\right)=\frac{2\left(1-3 \xi^{2}\right)}{\left(1+\xi^{2}\right)^{4}}, \quad \ell \geq 3 \\
K\left(\frac{\partial}{\partial x_{1}}, \frac{\partial}{\partial x_{2}}\right)=K\left(\frac{\partial}{\partial x_{r}}, \frac{\partial}{\partial x_{l}}\right)=0, \quad r, \ell \geq 3 .
\end{gathered}
$$

De fato, lembrando que podemos determinar a curvatura seccional pelos símbolos de christoffel,

$$
\bar{R}_{i j k \ell}=\bar{\Gamma}_{i k}^{s} \bar{\Gamma}_{j s}^{\ell}-\bar{\Gamma}_{j k}^{s} \bar{\Gamma}_{i s}^{\ell}+\frac{\partial}{\partial x_{j}} \bar{\Gamma}_{i k}^{\ell}-\frac{\partial}{\partial x_{i}} \bar{\Gamma}_{j k}^{\ell}
$$


Pelas expressões $(2.9),(2.10),(2.11)$ e (2.12) temos

$$
\begin{aligned}
& K\left(\frac{\partial}{\partial x_{1}}, \frac{\partial}{\partial x_{2}}\right)=\bar{R}_{1212}=\bar{\Gamma}_{11}^{\ell} \bar{\Gamma}_{2 \ell}^{2}-\bar{\Gamma}_{21}^{\ell} \bar{\Gamma}_{1 \ell}^{2}+\frac{\partial}{\partial x_{2}} \bar{\Gamma}_{11}^{2}-\frac{\partial}{\partial x_{1}} \bar{\Gamma}_{21}^{2} \\
& =\left(\frac{\varphi_{x_{1}}}{\varphi}\right)^{2}-\varepsilon_{1} \varepsilon_{2}\left(\frac{\varphi_{x_{2}}}{\varphi}\right)^{2}+\varepsilon_{1} \varepsilon_{2}\left(\frac{\varphi_{x_{2}}}{\varphi}\right)^{2}-\left(\frac{\varphi_{x_{1}}}{\varphi}\right)^{2} \\
& +\varepsilon_{1} \varepsilon_{2} \frac{\partial}{\partial x_{2}}\left(\frac{\varphi_{x_{2}}}{\varphi}\right)+\frac{\partial}{\partial x_{1}}\left(\frac{\varphi_{x_{1}}}{\varphi}\right)=0 \\
& K\left(\frac{\partial}{\partial x_{1}}, \frac{\partial}{\partial x_{\ell}}\right)=\bar{R}_{1 \ell 1 \ell}=\bar{\Gamma}_{11}^{s} \bar{\Gamma}_{\ell s}^{\ell}-\bar{\Gamma}_{\ell 1}^{s} \bar{\Gamma}_{1 s}^{\ell}+\frac{\partial}{\partial x_{\ell}} \bar{\Gamma}_{11}^{\ell}-\frac{\partial}{\partial x_{1}} \bar{\Gamma}_{\ell 1}^{\ell} \\
& =\bar{\Gamma}_{11}^{1} \bar{\Gamma}_{\ell 1}^{\ell}+\bar{\Gamma}_{11}^{2} \bar{\Gamma}_{\ell 2}^{\ell}-\left(\bar{\Gamma}_{\ell 1}^{\ell}\right)^{2}-\frac{\partial}{\partial_{x_{1}}} \bar{\Gamma}_{\ell 1}^{\ell} \\
& =\left(-\frac{\varphi_{x_{1}}}{\varphi}\right)\left(-\frac{\varphi_{x_{1}}}{\varphi}\right)+\left(\varepsilon_{1} \varepsilon_{2} \frac{\varphi_{x_{2}}}{\varphi}\right)\left(-\frac{\varphi_{x_{2}}}{\varphi}\right)-\left(-\frac{\varphi_{x_{1}}}{\varphi}\right)^{2}-\frac{\partial}{\partial_{x_{1}}}\left(-\frac{\varphi_{x_{1}}}{\varphi}\right) \\
& =\left(\frac{\varphi_{x_{1}}}{\varphi}\right)^{2}+\left(\frac{\varphi_{x_{2}}}{\varphi}\right)^{2}-\left(\frac{\varphi_{x_{1}}}{\varphi}\right)^{2}+\frac{\varphi_{x_{1} x_{1}}}{\varphi}-\left(\frac{\varphi_{x_{1}}}{\varphi}\right)^{2} \\
& =\frac{\varphi_{x_{1} x_{1}}}{\varphi}=\frac{-2\left(1-3 \xi^{2}\right)}{\left(1+\xi^{2}\right)^{2}}, \quad \ell \geq 3 \text {. } \\
& \begin{aligned}
K\left(\frac{\partial}{\partial x_{2}}, \frac{\partial}{\partial x_{\ell}}\right) & =\bar{R}_{2 \ell 2 \ell}=\bar{\Gamma}_{22}^{s} \bar{\Gamma}_{\ell s}^{\ell}-\bar{\Gamma}_{\ell 2}^{s} \bar{\Gamma}_{2 s}^{\ell}+\frac{\partial}{\partial x_{\ell}} \bar{\Gamma}_{22}^{\ell}-\frac{\partial}{\partial x_{2}} \bar{\Gamma}_{\ell 2}^{\ell} \\
& =\bar{\Gamma}_{22}^{1} \bar{\Gamma}_{\ell 1}^{\ell}+\bar{\Gamma}_{22}^{2} \bar{\Gamma}_{\ell 2}^{\ell}-\left(\bar{\Gamma}_{\ell 2}^{\ell}\right)^{2}-\frac{\partial}{\partial_{x_{2}}} \bar{\Gamma}_{\ell 2}^{\ell} \\
& =\left(\varepsilon_{1} \varepsilon_{2} \frac{\varphi_{x_{1}}}{\varphi}\right)\left(-\frac{\varphi_{x_{1}}}{\varphi}\right)+\left(-\frac{\varphi_{x_{2}}}{\varphi}\right)\left(-\frac{\varphi_{x_{2}}}{\varphi}\right)-\left(-\frac{\varphi_{x_{2}}}{\varphi}\right)^{2}-\frac{\partial}{\partial_{x_{2}}}\left(-\frac{\varphi_{x_{2}}}{\varphi}\right) \\
& =\frac{\varphi_{x_{2} x_{2}}}{\varphi}=\frac{-2\left(1-3 \xi^{2}\right)}{\left(1+\xi^{2}\right)^{2}}, \quad \ell \geq 3 .
\end{aligned} \\
& K\left(\frac{\partial}{\partial x_{r}}, \frac{\partial}{\partial x_{\ell}}\right)=\bar{R}_{r \ell r \ell}=\bar{\Gamma}_{r r}^{1} \bar{\Gamma}_{\ell 1}^{\ell}+\bar{\Gamma}_{r r}^{2} \bar{\Gamma}_{\ell 2}^{\ell} \\
& =\varepsilon_{1} \varepsilon_{3}\left(\frac{\varphi_{x_{1}}}{\varphi}\right)\left(\frac{-\varphi_{x_{1}}}{\varphi}\right)+\varepsilon_{2} \varepsilon_{3}\left(\frac{\varphi_{x_{2}}}{\varphi}\right)\left(\frac{-\varphi_{x_{2}}}{\varphi}\right)=0, \quad r, \ell \geq 3 .
\end{aligned}
$$

Portanto, podemos ver por (2.6) que o tensor de Ricci é dado por 
$R_{i j}=(n-2) \frac{\varphi_{x_{i} x_{j}}}{\varphi}=-2(n-2) \frac{1-3 \xi^{2}}{\left(1+\xi^{2}\right)^{2}}, \quad R_{r \ell}=(n-2) \frac{\varphi_{x_{r} x_{l}}}{\varphi}=0, \quad 1 \leq i, j \leq 2, \quad r, \ell \geq 3$

e, por (2.7), que sua curvatura escalar $R_{\bar{g}}=0$.

Agora vamos provar que esse soliton gradiente de Yamabe é completo, mostrando que qualquer geodésica $\phi\left(x_{1}(t), \ldots, x_{n}(t)\right)$ é definida para todo $t \in \mathbb{R}$. Já que $\nabla_{g} \varphi=$ $\sum_{i=1}^{n} \varepsilon_{i} \varphi_{x_{i}} \frac{\partial}{\partial_{x_{i}}}$, segue de $(2.22)$ que a curva $\phi$ é uma geodésica de $\left(\mathbb{R}^{n}, \bar{g}\right)$ se, e somente se, suas funções coordenadas safisfazem

$$
x_{i}^{\prime \prime}(t)=\frac{1}{\varphi}\left[2 \frac{d}{d t}(\varphi \circ \phi) x_{i}^{\prime}(t)-\varepsilon_{i} \alpha_{i} \varphi_{\xi} \sum_{k=1}^{n} \varepsilon_{k}\left(x_{k}^{\prime}(t)\right)^{2}\right], \quad 1 \leq i \leq n .
$$

Por (2.23) nós temos

$$
x_{\ell}^{\prime \prime}(t)=\frac{1}{\varphi}\left[2 \frac{d}{d t}(\varphi \circ \phi) x_{3}^{\prime}(t)\right]=\frac{-4 \xi \xi^{\prime}}{\left(1+\xi^{2}\right)} x_{\ell}^{\prime}, \quad \ell \geq 3 .
$$

Isso implica que

$$
\left[x_{\ell}^{\prime}(t)\left(1+\xi^{2}\right)^{2}\right]^{\prime}=0
$$

Logo,

$$
x_{\ell}^{\prime}(t)=\frac{\kappa_{\ell}}{\left(1+\xi^{2}\right)^{2}}, \quad \kappa_{\ell} \in \mathbb{R}
$$

Já que $\varphi_{\xi}=\frac{-2 \xi}{\left(1+\xi^{2}\right)^{2}}$ por $(2.23)$ e $(2.24)$ teremos que

$$
\begin{aligned}
x_{1}^{\prime \prime}(t) & =\frac{1}{\varphi}\left[2 \frac{d}{d t}(\varphi \circ \phi) x_{1}^{\prime}(t)-\varepsilon_{1} \alpha_{1} \varphi_{\xi} \sum_{k=1}^{n} \varepsilon_{k}\left(x_{k}^{\prime}(t)\right)^{2}\right] \\
& =\left[\frac{-4 \xi \xi^{\prime}}{1+\xi^{2}} x_{1}^{\prime}-\frac{2 \xi}{1+\xi^{2}}\left(\left(x_{1}^{\prime}\right)^{2}+\left(x_{2}^{\prime}\right)^{2}+\sum_{\ell=3}^{n}\left(\frac{\kappa_{\ell}}{\left(1+\xi^{2}\right)^{2}}\right)^{2}\right)\right] \\
& =-\frac{2 \xi\left(\xi^{\prime}\right)^{2}}{1+\xi^{2}}-\frac{2 \xi \bar{\sigma}}{\left(1+\xi^{2}\right)^{5}} .
\end{aligned}
$$


onde $\bar{\sigma}=\sum_{\ell=3}^{n} \kappa_{\ell}^{2}$. Analogamente obtemos

$$
x_{2}^{\prime \prime}(t)=-\frac{2 \xi\left(\xi^{\prime}\right)^{2}}{1+\xi^{2}}+\frac{2 \xi \bar{\sigma}}{\left(1+\xi^{2}\right)^{5}} .
$$

Portanto, por (2.25) e (2.26) obtemos

$$
\xi^{\prime \prime}+\frac{4 \xi\left(\xi^{\prime}\right)^{2}}{1+\xi^{2}}=0
$$

e então

$$
\xi^{\prime}=\frac{\bar{\kappa}}{\left(1+\xi^{2}\right)^{2}}, \quad \bar{\kappa} \in \mathbb{R} .
$$

Por $(2.25),(2.26)$ e (2.27) nós temos

$$
\left\{\begin{array}{l}
x_{1}^{\prime \prime}(t)=\frac{-2\left(\bar{\sigma}+\bar{\kappa}^{2}\right)\left(x_{1}+x_{2}\right)}{\left(1+\left(x_{1}+x_{2}\right)^{2}\right)^{5}}, \\
x_{2}^{\prime \prime}(t)=\frac{2\left(\bar{\sigma}-\bar{\kappa}^{2}\right)\left(x_{1}+x_{2}\right)}{\left(1+\left(x_{1}+x_{2}\right)^{2}\right)^{5}}
\end{array}\right.
$$

Observe que a função $f(x, y)=\frac{x+y}{\left[1+(x+y)^{2}\right]^{5}}$ é diferenciável em todo ponto. Logo, pela desigualdade do valor médio, nós temos que $f(x, y)$ é Lipchistizian. De fato,

$$
|\nabla f|=\sqrt{\left(\frac{1-9(x+y)^{2}}{\left[1+(x+y)^{2}\right]^{6}}\right)^{2}+\left(\frac{1-9(x+y)^{2}}{\left[1+(x+y)^{2}\right]^{6}}\right)^{2}} \leq \frac{9 \sqrt{2}\left(1+(x+y)^{2}\right)}{\left[1+(x+y)^{2}\right]^{6}} \leq 9 \sqrt{2} .
$$

Portanto,

$$
\left|f\left(x_{1}, x_{2}\right)-f\left(x_{3}, x_{4}\right)\right| \leq 9 \sqrt{2}\left|\left(x_{1}, x_{2}\right)-\left(x_{3}, x_{4}\right)\right|,
$$


isto é, $f$ é Lipchistizian. Agora, por (2.24) e (2.28)

$$
\left\{\begin{array}{l}
x_{1}^{\prime}=y_{1} \\
x_{2}^{\prime}=y_{2} \\
y_{1}^{\prime}=-2 \xi \frac{\left(\bar{\kappa}^{2}+\bar{\sigma}\right)}{\left(1+\xi^{2}\right)^{5}} \quad \text { onde } \quad \xi=x_{1}+x_{2} . \\
y_{2}^{\prime}=2 \xi \frac{\left(\bar{\sigma}-\bar{\kappa}^{2}\right)}{\left(1+\xi^{2}\right)^{5}} \\
x_{\ell}^{\prime}=\frac{\kappa}{\left(1+\xi^{2}\right)^{2}}, \quad \ell \geq 3
\end{array}\right.
$$

Já que $g(x, y)=\frac{1}{\left(1+(x+y)^{2}\right)^{2}}$ também é Lipchistizian. De fato,

$$
|\nabla g|=\sqrt{\left(\frac{-4(x+y)}{\left[1+(x+y)^{2}\right]^{3}}\right)^{2}+\left(\frac{-4(x+y)}{\left[1+(x+y)^{2}\right]^{3}}\right)^{2}} \leq \sqrt{\frac{32\left(1+(x+y)^{2}\right)}{\left[1+(x+y)^{2}\right]^{6}}} \leq 4 \sqrt{2} .
$$

Finalmente, as soluções do sistema existem para todo $t \in \mathbb{R}$ e, portanto, as geodésicas $\phi(t)$ estão definidas para $t \in \mathbb{R}$, isto é, a variedade é completa.

Os resultados desse capítulo podem ser encontrados em [45]. 


\section{Capítulo 3}

\section{Soliton gradiente quasi Yamabe em dimensão $n=4$}

Nesse capítulo, vamos mostrar que a condição localmente conformemente plana no Teorema 1.30, obtido em [15], e no Teorema 1.28, obtido em [30], pode ser substituída pela condição mais fraca semi localmente conformemente fraca ((anti-)self dual tensor de Weyl) no caso de dimensão $n=4$, isto é, $W^{+}=0$ (veja as Seções 1.1 e 1.6). Motivados por [23] e [30], consideramos os solitons quasi Yamabe gradientes de dimensão quatro.

Em [25], Daskalopoulos e Sesum provaram que solitons de Yamabe com curvatura seccional positiva são rotacionalmente simétricos, com a hipótese adicional de que a métrica $g$ é localmente conformemente plana. Cao, Sun e Zhang [15] demonstraram que os solitons de Yamabe possuem uma estrutura especial de produto torcido, sem nenhuma condição adicional.

\subsection{Preliminares}

Antes de mais nada, vamos relembrar a equação de Ricci,

$$
\nabla_{i} \nabla_{j} \nabla_{k} f-\nabla_{j} \nabla_{i} \nabla_{k} f=R_{i j k l} \nabla^{l} f
$$

Veremos alguns resultados preliminares que são muito importantes para o nosso trabalho, e por completude incluiremos suas demonstrações. 
Lema 3.1. [30] Seja $\left(M^{n}, g, f\right)$ um soliton gradiente quasi Yamabe completo e não trivial satisfazendo (1.17). Então,

$$
\begin{gathered}
n(R-\lambda)=\Delta f-\frac{1}{m}|\nabla f|^{2}, \\
\nabla_{i}|\nabla f|^{2}=2(R-\lambda) \nabla_{i} f+\frac{2}{m}|\nabla f|^{2} \nabla_{i} f
\end{gathered}
$$

e

$$
\nabla_{i} R=\frac{1}{m}(R-\lambda) \nabla_{i} f-\frac{1}{n-1} R_{i j} \nabla^{j} f
$$

onde $R_{i j}$ é o tensor de Ricci da métrica e $\nabla^{j} f=g^{k j} \nabla_{k} f$.

Demonstração. Para demonstrarmos a equação (3.2) basta fazer a contração da equação (1.17). De fato,

$$
n(R-\lambda)=g^{i j} \nabla_{i} \nabla_{j} f-\frac{1}{m} g^{i j} \nabla_{i} f \nabla_{j} f
$$

A equação (3.3) é também um resultado direto de (1.17). Note que,

$$
\begin{aligned}
\nabla_{i}|\nabla f|^{2} & =2 \nabla_{i} \nabla_{j} f \nabla^{j} f=2\left[(R-\lambda) g_{i j}+\frac{1}{m} \nabla_{i} f \nabla_{j} f\right] \nabla^{j} f \\
& =2(R-\lambda) \nabla_{i} f+\frac{2}{m}|\nabla f|^{2} \nabla_{i} f .
\end{aligned}
$$

Por último, para provarmos a equação (3.4) calcular o divergente da equação (1.17), então usando as equações anteriores, a identidade de Ricci e a dupla contração da identidade de Bianchi. Entenda por divergente como tomar a derivada covariante da equação (1.17) e em seguida fazer a contração da mesma.

$$
\nabla_{l}(R-\lambda) g_{i j}=\nabla_{l} \nabla_{i} \nabla_{j} f-\frac{1}{m}\left[\left(\nabla_{l} \nabla_{i} f\right) \nabla_{j} f+\left(\nabla_{l} \nabla_{j} f\right) \nabla_{i} f\right] .
$$

Agora, usamos a identidade de Ricci para obtermos

$$
\nabla_{l}(R-\lambda) g_{i j}=\nabla_{i} \nabla_{l} \nabla_{j} f-R_{i l j s} \nabla^{s} f-\frac{1}{m}\left[\left(\nabla_{l} \nabla_{i} f\right) \nabla_{j} f+\left(\nabla_{l} \nabla_{j} f\right) \nabla_{i} f\right] .
$$

Contraindo em $l$ e $j$ a equação acima temos

$$
g^{j l} \nabla_{l} R g_{i j}=g^{j l} \nabla_{i} \nabla_{l} \nabla_{j} f-g^{j l} R_{i l j s} \nabla^{s} f-\frac{1}{m}\left[g^{j l}\left(\nabla_{l} \nabla_{i} f\right) \nabla_{j} f+g^{j l}\left(\nabla_{l} \nabla_{j} f\right) \nabla_{i} f\right],
$$


portanto,

$$
\begin{aligned}
\nabla_{i} R & =\nabla_{i}(\Delta f)+R_{i s} \nabla^{s} f-\frac{1}{m}\left[\nabla_{l} \nabla_{i} f \nabla^{l} f+(\Delta f) \nabla_{i} f\right] \\
& =\nabla_{i}(\Delta f)+R_{i s} \nabla^{s} f-\frac{1}{2 m} \nabla_{i}|\nabla f|^{2}-\frac{1}{m}(\Delta f) \nabla_{i} f
\end{aligned}
$$

Se usarmos, na identidade acima, a equação (3.2) teremos que

$$
\begin{aligned}
\nabla_{i} R & =\nabla_{i}\left(n(R-\lambda)+\frac{1}{m}|\nabla f|^{2}\right)+R_{i s} \nabla^{s} f-\frac{1}{2 m} \nabla_{i}|\nabla f|^{2}-\frac{1}{m}\left(n(R-\lambda)+\frac{1}{m}|\nabla f|^{2}\right) \nabla_{i} f \\
& =n \nabla_{i} R+\frac{1}{m} \nabla_{i}|\nabla f|^{2}+R_{i s} \nabla^{s} f-\frac{1}{2 m} \nabla_{i}|\nabla f|^{2}-\frac{n}{m}(R-\lambda) \nabla_{i} f-\frac{1}{m^{2}}|\nabla f|^{2} \nabla_{i} f \\
& =n \nabla_{i} R+\frac{1}{2 m} \nabla_{i}|\nabla f|^{2}+R_{i s} \nabla^{s} f-\frac{n}{m}(R-\lambda) \nabla_{i} f-\frac{1}{m^{2}}|\nabla f|^{2} \nabla_{i} f .
\end{aligned}
$$

Por último, usamos a equação (3.3) na equação acima para obter

$$
\begin{aligned}
\nabla_{i} R & =n \nabla_{i} R+\frac{1}{2 m}\left(2(R-\lambda) \nabla_{i} f+\frac{2}{m}|\nabla f|^{2} \nabla_{i} f\right)+R_{i s} \nabla^{s} f-\frac{n}{m}(R-\lambda) \nabla_{i} f-\frac{1}{m^{2}}|\nabla f|^{2} \nabla_{i} f \\
& =n \nabla_{i} R+R_{i s} \nabla^{s} f-\frac{(n-1)}{m}(R-\lambda) \nabla_{i} f
\end{aligned}
$$

Com essa última equação concluimos a demonstração do lema.

Como conseqüência do Lema 3.1 temos o resultado abaixo, ele é a ponte entre o tensor de Weyl e a métrica do soliton quasi Yamabe gradiente.

Proposição 3.2. [30] Seja $\left(M^{n}, g, f\right)$ um soliton gradiente quasi Yamabe completo e não trivial satisfazendo (1.17). Então

$$
D_{i j k}=W_{i j k l} \nabla^{l} f
$$

Demonstração. Note que, derivando a equação (1.17) temos que

$$
\begin{aligned}
\nabla_{i} \nabla_{j} \nabla_{k} f-\nabla_{j} \nabla_{i} \nabla_{k} f & =\nabla_{i}\left((R-\lambda) g_{k j}+\frac{1}{m} \nabla_{k} f \nabla_{j} f\right)-\nabla_{j}\left((R-\lambda) g_{i k}+\frac{1}{m} \nabla_{i} f \nabla_{k} f\right) \\
& =\nabla_{i} R g_{k j}+\frac{1}{m} \nabla_{i}\left(\nabla_{k} f \nabla_{j} f\right)-\nabla_{j} R g_{i k}+\frac{1}{m} \nabla_{j}\left(\nabla_{i} f \nabla_{k} f\right) \\
& =\nabla_{i} R g_{k j}-\nabla_{j} R g_{i k}+\frac{1}{m}\left(\nabla_{j} f \nabla_{i} \nabla_{k} f-\nabla_{i} f \nabla_{j} \nabla_{k} f\right) .
\end{aligned}
$$


Pela equação (1.17) novamente, obtemos

$$
\begin{aligned}
\nabla_{i} \nabla_{j} \nabla_{k} f-\nabla_{j} \nabla_{i} \nabla_{k} f & =\nabla_{i} R g_{k j}-\nabla_{j} R g_{i k}+\frac{1}{m}\left(\nabla_{j} f \nabla_{i} \nabla_{k} f-\nabla_{i} f \nabla_{j} \nabla_{k} f\right) \\
& =\nabla_{i} R g_{k j}-\nabla_{j} R g_{i k}-\frac{(R-\lambda)}{m}\left(g_{k j} \nabla_{i} f-g_{k i} \nabla_{j} f\right)
\end{aligned}
$$

Usando a equação (3.4) temos diretamente que

$$
\nabla_{i} \nabla_{j} \nabla_{k} f-\nabla_{j} \nabla_{i} \nabla_{k} f=-\frac{1}{n-1}\left(g_{j k} R_{i l} \nabla^{l} f-g_{i k} R_{j l} \nabla^{l} f\right) .
$$

Por outro lado, a identidade de Ricci e o tensor de Weyl nos garante que

$$
\begin{aligned}
\nabla_{i} \nabla_{j} \nabla_{k} f-\nabla_{j} \nabla_{i} \nabla_{k} f & =R_{i j k l} \nabla^{l} f \\
& =W_{i k j l} \nabla^{l} f-\frac{R}{(n-1)(n-2)}\left(g_{j l} g_{i k}-g_{i l} g_{j k}\right) \nabla^{l} f \\
& +\frac{1}{n-2}\left(R_{i k} g_{j l}+R_{j l} g_{i k}-R_{i l} g_{j k}-R_{j k} g_{i l}\right) \nabla^{l} f \\
& =W_{i k j l} \nabla^{l} f+\frac{R}{(n-1)(n-2)}\left(g_{j l} g_{i k}-g_{i l} g_{j k}\right) \nabla^{l} f \\
& -\frac{1}{n-2}\left(R_{j k} \nabla_{i} f-R_{i k} \nabla_{j} f\right)-\frac{1}{n-2}\left(R_{i l} \nabla^{l} f g_{j k}-R_{j l} \nabla^{l} f g_{i k}\right) .
\end{aligned}
$$

Portanto, pela definição do tensor $D,(3.6)$ e (3.7) temos que

$$
\begin{aligned}
D_{i j k} & =\frac{1}{n-2}\left(R_{j k} \nabla_{i} f-R_{i k} \nabla_{j} f\right)+\frac{1}{(n-1)(n-2)}\left(R_{i l} \nabla^{l} f g_{j k}-R_{j l} \nabla^{l} f g_{i k}\right) \\
& -\frac{R}{(n-1)(n-2)}\left(g_{j k} \nabla_{i} f-g_{i k} \nabla_{j} f\right)=W_{i k j l} \nabla^{l} f .
\end{aligned}
$$

O que conclui a prova da proposição.

O próximo lema é o principal resultado técnico do capítulo, que será usado para demonstrar os Teoremas 3.6 e 3.8 .

Lema 3.3. Seja $\left(M^{4}, g, f\right)$ um anti-self dual (ou self dual) soliton gradiente quasi Yamabe completo e não-trivial satisfazendo (1.17). Então o tensor $D \equiv 0$.

Demonstração. Para a demonstração desse resultado, vamos seguir as idéias em [23] (ver também [4]). Seja uma variedade 4-dimensional Riemanniana satisfazendo a Definição 1.27 tal que $W^{+}=0$, isto é, um anti-self dual soliton quasi Yamabe gradiente completo. Portanto, por (1.25) temos que

$$
W_{i j k l}+W_{\bar{i} \bar{j} k l}=0 .
$$


Logo,

$$
W_{i j k l} \nabla^{l} f+W_{\bar{i} \bar{j} k l} \nabla^{l} f=0 .
$$

Isso implica, pela Proposição 3.2, que

$$
D_{i j k}+D_{\bar{i} \bar{j} k}=0
$$

Agora, considerando um referencial ortonormal orientado $\left\{e_{i}\right\}_{i=1}^{4}$ que diagonaliza o tensor de Ricci, com autovalores associados a base $\mu_{k}, k=1, \ldots, 4$, respectivamente, isto é, $R_{i j}=\mu_{i} \delta_{i j}$, onde $\delta_{i j}$ é o delta de Kronecker. Temos por (1.19)

$$
D_{12 k}+D_{34 k}=0, \quad D_{13 k}+D_{42 k}=0 \quad \text { e } \quad D_{14 k}+D_{23 k}=0 .
$$

Pela definição do tensor (1.13) temos

$$
\begin{aligned}
D_{i j k} & =\frac{1}{n-2}\left(R_{j k} \nabla_{i} f-R_{i k} \nabla_{j} f\right)+\frac{1}{(n-1)(n-2)}\left(R_{i l} \nabla^{l} f g_{j k}-R_{j l} \nabla^{l} f g_{i k}\right) \\
& -\frac{R}{(n-1)(n-2)}\left(g_{j k} \nabla_{i} f-g_{i k} \nabla_{j} f\right) \\
& =\frac{1}{n-2}\left(\mu_{j} \delta_{j k} \nabla_{i} f-\mu_{i} \delta_{i k} \nabla_{j} f\right)+\frac{1}{(n-1)(n-2)}\left(\mu_{i} \nabla_{i} f \delta_{j k}-\mu_{j} \nabla_{j} f \delta_{i k}\right) \\
& -\frac{R}{(n-1)(n-2)}\left(\delta_{j k} \nabla_{i} f-\delta_{i k} \nabla_{j} f\right) .
\end{aligned}
$$

Isso nos garante que no ponto $p$

$$
D_{i j k}=0, \quad \text { for } \quad i \neq j \neq k \text {. }
$$

Portanto, por (3.8) e (3.11) obtemos

$$
D_{i j i}=0, \quad \text { for } \quad i, j=1, \ldots, 4 \text {. }
$$

Como também já vimos o tensor $D$ é anti-simétrico, isto é, $D_{i i j}=0$. Isto garante que $D \equiv 0$, como queríamos demonstrar.

A demonstração do Teorema 3.8 é uma conseqüência direta do Lema 3.3. Entretanto, para aplicarmos o lema, precisamos entender como é a estrutura de produto torcido no soliton gradiente de Yamabe. Essa análise foi feita, anteriormente, por [25] com a condição adicional da métrica ser localmente conformemente plana. Aqui, vamos seguir os passos de [15]. Cao, Sun e Zhang [15] estudaram a estrutura do produto torcido 
nos solitons gradientes de Yamabe completo sem nenhuma hipótese adicional sobre a variedade ou a métrica.

Seja $\left(M^{n}, g, f\right)$ um soliton gradiente de Yamabe completo não trivial, satisfazendo (1.16). Para um valor regular qualquer $c$ da função potencial $f$, considere os conjuntos de nível $\Sigma_{c}=f^{-1}(c)$. Suponha que $I$ seja um intervalo aberto contendo $c$, tal que $f$ não tenha pontos críticos em $I$. Seja $U_{I}=f^{-1}(I)$. Numa vizinhança qualquer dos conjuntos de nível, onde $\nabla f \neq 0$, podemos escrever a métrica, em $U_{I}$, como

$$
d s^{2}=\frac{1}{|\nabla f|^{2}} d f^{2}+\bar{g}_{\Sigma_{c}}
$$

$\forall p \in U_{I}, \bar{g}_{\Sigma_{f(p)}}=g_{a b}(f(p), \theta) d \theta_{a} d \theta_{b}$ é a métrica induzida e $\theta=\left(\theta_{2}, \ldots, \theta_{n}\right)$ é um sistema de coordenadas locais em $\Sigma_{f(p)}$. Vamos considerar $a, b, c, d \in\{(2, \ldots, n)\}$.

Por outro lado, como já foi mostrado em [25], temos que

$$
\nabla_{1}|\nabla f|^{2}=2 \nabla^{2} f(\nabla f, \nabla f)=2(R-\lambda)|\nabla f|
$$

e

$$
\nabla_{a}|\nabla f|^{2}=2 \nabla^{2} f\left(\nabla f, \partial_{a}\right)=2(R-\lambda) \nabla_{a} f=0
$$

Portanto, $|\nabla f|^{2}$ só depende da variável $f(p), \forall p \in U_{I}$. Isso nos permite fazer uma mudança de coordenadas,

$$
r(x)=\int \frac{d f}{|\nabla f|}
$$

de tal forma que podemos escrever a métrica em $U_{I}$ como

$$
d s^{2}=d r^{2}+g_{a b}(r, \theta) d \theta_{a} d \theta_{b}
$$

Seja $\nabla r=\frac{\partial}{\partial r}$, então, pelo Lema 1.35, $|\nabla r|=1$ e $\nabla f=f^{\prime}(r) \nabla r$ em $U_{I}$. Repare que $f^{\prime}(r)$ em $U_{I}$ não muda de sinal, já que $f$ não possui pontos críticos nesse aberto. Assim, podemos assumir que $I=(\alpha, \beta)$ com $f^{\prime}(r)>0$ para todo $r \in I$. Como

$$
\nabla_{\partial r} \partial_{r}=0
$$

então as curvas integrais a $\nabla r$ são geodésicas normais. 
Por (3.13), a equação (1.16) nos dá

$$
R-\lambda=\nabla^{2} f\left(\frac{\partial}{\partial r}, \frac{\partial}{\partial r}\right)=f^{\prime \prime}(r)
$$

Portanto, podemos concluir diretamente que a curvatura escalar só depende de $f(p)$ em $U_{I}$. Além disso, a segunda forma fundamental de $\Sigma_{f(p)}$ é dada por

$$
h_{a b}=\frac{\nabla_{a} \nabla_{b} f}{|\nabla f|}=\frac{f^{\prime \prime}(r)}{f^{\prime}(r)} g_{a b}, \quad a, b \in\{2, \ldots, n\} .
$$

Em particular,

$$
H_{f(p)}=(n-1) \frac{f^{\prime \prime}(r)}{f^{\prime}(r)}
$$

Ou seja, pontos de $\Sigma_{f(p)}$ são todos umbílicos pois $h_{a b}=\frac{H_{f(p)}}{(n-1)} g_{a b}$. E portanto, $H_{f(p)}$ é constante em $\Sigma_{f(p)}$ (veja Capítulo 5 para detalhes sobre os cálculos feitos com a segunda forma fundamental e a curvatura média).

Fixando um sistema de coordenadas

$$
\left(r, \theta_{2}, \ldots, \theta_{n}\right)
$$

em $U_{I}$, onde $\left(\theta_{2}, \ldots, \theta_{n}\right)$ é um sistema de coordenadas locais em $\Sigma_{c}$, lembrando que $a, b, c, d \in\{2, \ldots, n\}$. Então, nesse sistema de coordenadas locais temos que

$$
h_{a b}=-g\left(\partial_{r}, \nabla_{a} \partial_{b}\right)=-g\left(\partial_{r}, \Gamma_{a b}^{1} \partial_{r}\right)=-\Gamma_{a b}^{1}
$$

Por outro lado, por (1.2) temos que

$$
\Gamma_{a b}^{1}=\frac{1}{2} g^{11}\left(-\frac{\partial g_{a b}}{\partial r}\right)=-\frac{1}{2} \frac{\partial g_{a b}}{\partial r} .
$$

Portanto, por (3.14) temos

$$
\frac{\partial g_{a b}}{\partial r}=2 \frac{f^{\prime \prime}(r)}{f^{\prime}(r)} g_{a b}
$$


Como

$$
\begin{aligned}
0 & =\frac{1}{\left(f^{\prime}(r)\right)^{2}}\left(\frac{\partial}{\partial r} g_{a b}-2 \frac{f^{\prime \prime}(r)}{f^{\prime}(r)} g_{a b}\right) \\
& =\frac{1}{\left(f^{\prime}(r)\right)^{4}}\left(\left(f^{\prime}(r)\right)^{2} \frac{\partial}{\partial r} g_{a b}-2 f^{\prime \prime}(r) f^{\prime}(r) g_{a b}\right) \\
& =\frac{\partial}{\partial r}\left(\frac{g_{a b}}{f^{\prime}(r)^{2}}\right) .
\end{aligned}
$$

Isso implica que,

$$
g_{a b}(r, \theta)=f^{\prime}(r)^{2} g_{a b}\left(r_{0}, \theta\right)
$$

onde o conjunto de nível $\left\{r=r_{0}\right\}$ corresponde a $\Sigma_{c_{0}}=f^{-1}\left(c_{0}\right)$. Para qualquer valor regular $c_{0}$ da função potencial $f$.

Essa discussão, nos permite enunciar a seguinte proposição:

Proposição 3.4. [15] Seja $\left(M^{n}, g, f\right)$ um soliton gradiente de Yamabe completo não trivial satisfazendo (1.16) e $\Sigma_{c}=f^{-1}(c)$ uma superfície de nivel regular. Então

1. $|\nabla f|^{2}$ é constante em $\Sigma_{c}$,

2. a curvatura escalar $R$ é constante em $\Sigma_{c}$,

3. a segunda forma fundamental em $\Sigma_{c}$ é dada por $h_{a b}=\frac{H}{n-1} g_{a b}$,

4. a curvatura média $H$ é constante em $\Sigma_{c}$,

5. em uma vizinhança aberta $U$ de $\Sigma_{c}$ tal que $f$ não tenha pontos críticos, a métrica $g$ pode ser expressa como

$$
d s^{2}=d r^{2}+\left(f^{\prime}(r)\right)^{2} \bar{g}_{r_{0}},
$$

onde $\left(\theta_{2}, \ldots, \theta_{n}\right)$ é um sistema de coordenadas locais em $\Sigma_{c}$ e $\bar{g}_{r_{0}}=g_{a b}\left(r_{0}, \theta\right) d \theta_{a} d \theta_{b}$ é a métrica induzida em $\Sigma_{c}=r^{-1}\left(r_{0}\right)$.

Observação 3.5. Esta proposição foi primeiramente observada em [25] com a condição adicional da métrica ser localmente conformemente plana.

Motivados pela Proposição 3.4 acima, estudaremos o tensor curvatura e o tensor de Ricci da variedade produto

$$
\left(M^{n}, g\right)=\left(I, d r^{2}\right) \times \varphi\left(N^{n-1}, \bar{g}\right)
$$


onde $d s^{2}=d r^{2}+\varphi^{2}(r) \bar{g}$. Fixando um sistema de coordenadas qualquer $\theta=\left(\theta_{2}, \ldots, \theta_{n}\right)$ em $N^{n-1}$, e escolhendo $\left(x_{1}, \ldots, x_{n}\right)=\left(r, \theta_{2}, \ldots, \theta_{n}\right)$ como em (3.15). Lembrando que $a, b, c, d \in\{2, \ldots, n\}$ e que o tensor curvatura $\bar{R}_{a b c d}$ é o tensor curvatura em $\left(N^{n-1}, \bar{g}\right)$. Portanto, pela Proposição 1.37 temos que

$$
R_{1 a 1 b}=-\varphi \varphi^{\prime \prime} \bar{g}_{a b}, \quad R_{1 a b c}=0
$$

e

$$
R_{a b c d}=\varphi^{2} \bar{R}_{a b c d}-\left(\varphi \varphi^{\prime}\right)^{2}\left(\bar{g}_{a c} \bar{g}_{b d}-\bar{g}_{a d} \bar{g}_{b c}\right)
$$

A Proposição 1.38 nos dá que o tensor de Ricci de $\left(M^{n}, g\right)$ é dado por

$$
R_{11}=-(n-1) \frac{\varphi^{\prime \prime}}{\varphi}, \quad R_{1 a}=0 \quad(2 \leq a \leq n)
$$

e

$$
R_{a b}=\bar{R}_{a b}-\left[(n-2)\left(\varphi^{\prime}\right)^{2}+\varphi \varphi^{\prime \prime}\right] \bar{g}_{a b}, \quad(2 \leq a, b \leq n)
$$

Então, contraíndo o tensor de Ricci, as curvaturas escalares de $\left(M^{n}, g\right)$ e $\left(N^{n-1}, \bar{g}\right)$ se relacionam pela seguinte expressão:

$$
R=\varphi^{-2} \bar{R}-(n-1)(n-2)\left(\frac{\varphi^{\prime}}{\varphi}\right)^{2}-2(n-1) \frac{\varphi^{\prime \prime}}{\varphi} .
$$

Usando as expressões (3.16), (3.17), (3.18), (3.19), (3.20) e (1.8) temos que o tensor de Weyl para a métrica produto da variedade $\left(M^{n}, g\right)$ é dado por:

$$
\begin{aligned}
W_{1 a 1 b} & =R_{1 a 1 b}-\frac{1}{n-2}\left(R_{11} g_{a b}+R_{a b} g_{11}-R_{1 b} g_{a 1}-R_{a 1} g_{1 b}\right)+\frac{R}{(n-1)(n-2)}\left(g_{a b} g_{11}-g_{1 b} g_{a 1}\right) \\
& =-\varphi \varphi^{\prime \prime} \bar{g}_{a b}-\frac{1}{(n-2)}\left\{-(n-1) \varphi \varphi^{\prime \prime} \bar{g}_{a b}+\bar{R}_{a b}-\left[(n-2)\left(\varphi^{\prime}\right)^{2}+\varphi \varphi^{\prime \prime}\right] \bar{g}_{a b}\right\} \\
& +\frac{1}{(n-1)(n-2)}\left[\bar{R}-(n-1)(n-2)\left(\varphi^{\prime}\right)^{2}-2(n-1) \varphi \varphi^{\prime \prime}\right] \bar{g}_{a b} \\
& =-\varphi \varphi^{\prime \prime} \bar{g}_{a b}+\frac{(n-1)}{(n-2)} \varphi \varphi^{\prime \prime} \bar{g}_{a b}-\frac{1}{(n-2)} \bar{R}_{a b}+\left(\varphi^{\prime}\right)^{2} \bar{g}_{a b}+\frac{1}{(n-2)} \varphi \varphi^{\prime \prime} \bar{g}_{a b} \\
& +\frac{\bar{R}}{(n-1)(n-2)} \bar{g}_{a b}-\left(\varphi^{\prime}\right)^{2} \bar{g}_{a b}-\frac{2}{(n-2)} \varphi \varphi^{\prime \prime} \bar{g}_{a b} \\
& =\frac{\bar{R}}{(n-1)(n-2)} \bar{g}_{a b}-\frac{\bar{R}_{a b}}{(n-2)},
\end{aligned}
$$




$$
W_{1 a b c}=0 \quad \text { e } \quad W_{a b c d}=\varphi^{2} \bar{W}_{a b c d}
$$

onde $\bar{W}$ é o tensor de Weyl de $\left(N^{n-1}, \bar{g}\right)$.

\subsection{Resultados principais}

Teorema 3.6. Seja $\left(M^{4}, g, f\right)$ um anti-self dual (ou self dual) soliton gradiente quasi Yamabe completo e não trivial satisfazendo (1.17) com curvatura seccional positiva. Então $\left(M^{4}, g, f\right)$ é rotacionalmente simétrico.

Demonstração. A demonstração segue diretamente do Lema 3.3 e do Teorema 1.28, da Seção 1.4 .

Analogamente temos o seguinte:

Corolário 3.7. Seja $\left(M^{4}, g, f\right)$ um anti-self dual (ou self dual) soliton gradiente de $Y a$ mabe completo e não trivial satisfazendo (1.16) com curvatura seccional positiva. Então $\left(M^{4}, g, f\right)$ é rotacionalmente simétrico.

$\mathrm{Na}$ verdade, temos o seguinte resultado.

Teorema 3.8. Seja $\left(M^{4}, g, f\right)$ um anti-self dual (ou self dual) soliton gradiente de Yamabe completo satisfazendo (1.16) e suponha que $f$ não tenha pontos críticos. Então $\left(M^{4}, g, f\right)$ é o produto torcido

$$
\left(\mathbb{R}, d r^{2}\right) \times|\nabla f|\left(N^{3}, \bar{g}\right),
$$

onde $\left(N^{3}, \bar{g}\right)$ é um espaço de curvatura seccional constante.

Demonstração. Seja $\left(M^{4}, g, f\right)$ um anti-self dual soliton gradiente de Yamabe, completo e não trivial tal que $f$ não tem pontos críticos. Pelo Teorema 1.29-(ii) temos que $\left(M^{4}, g, f\right)$ tem uma estrutura de produto torcido (1.18). Queremos demonstrar, na verdade, que $\left(N^{3}, \bar{g}\right)$ é um espaço de curvatura seccional constante. Para isso, é suficiente que $\bar{g}$ seja Einstein. Já que, variedades de Einstein tridimensionais tem curvatura seccional constante. Para concluir o resultado precisamos conhecer a estrutura de produto torcido do soliton gradiente de Yamabe, discutida nas preliminares. 
Pelo Lema 3.3 e pela Proposição 3.2 sabemos que $W_{i j k l} \nabla^{l} f=0$, isto é,

$$
W(\cdot, \cdot, \cdot, \nabla f)=0
$$

Considerando as superfícies de nível $\Sigma_{c}=f^{-1}(c)$ onde $c$ é qualquer valor regular para $f$. Supondo $I$ um intervalo aberto contendo $c$ tal que $f$ não tenha pontos críticos na vizinhança $U_{I}=f^{-1}(I)$. Já que $|\nabla f|^{2}$ é constante em $\Sigma_{c}$ (ver [25]), nós podemos fazer uma mudança de variáveis como em (3.12), e assim podemos aplicar a Proposição 3.4. Isto é, fixado um sistema de coordenadas qualquer $\theta=\left(\theta_{2}, \theta_{3}, \theta_{4}\right)$ em $N^{3}$, e escolhendo $\left(x_{1}, x_{2}, x_{3}, x_{4}\right)=\left(r, \theta_{2}, \theta_{3}, \theta_{4}\right)$. Sendo $\nabla r=\frac{\partial}{\partial r}$, então $|\nabla r|=1$ e $\nabla f=f^{\prime}(r) \frac{\partial}{\partial r}$ em $U_{I}$, como foi visto na seção preliminar desse capítulo. Portanto, temos por (3.22) que

$$
0=W(\cdot, \cdot, \cdot, \nabla f)=f^{\prime}(r) W(\cdot, \cdot, \cdot, \nabla r)
$$

Já que $f$ não possui nenhum ponto crítico

$$
W(\cdot, \cdot, \cdot, \nabla r)=0
$$

Logo, pelos argumentos sobre a variedade produto $\left(M^{n}, g\right)=\left(I, d r^{2}\right) \times \varphi\left(N^{n-1}, \bar{g}\right)$ vistos logo acima, a fórmula do tensor de Weyl (3.21) para essa variedade produto nos dá

$$
2 W\left(\nabla r, \partial_{a}, \nabla r, \partial_{b}\right)=\frac{\bar{R}}{3} \bar{g}\left(\partial_{a}, \partial_{b}\right)-\bar{R} i c\left(\partial_{a}, \partial_{b}\right), \quad \text { para } \quad a, b \in\{2,3,4\}
$$

onde $\bar{R}$ e $\bar{R} i c$ são, respectivamente, a curvatura escalar e o tensor de Ricci para $\left(N^{3}, \bar{g}\right)$. Finalmente, por (3.23) e (3.24) temos que $\bar{g}$ é Einstein. Logo, o Teorema 3.8 está demonstrado.

Os resultados desse capítulo podem ser encontrados em [46]. 


\section{Capítulo 4}

\section{Estudo da função potencial da métrica CPE}

Faremos aqui uma análise sobre a função potencial da métrica CPE (definida na Seção 1.7). Foi conjecturado que a métrica CPE é sempre Einstein (ver na seção 1.7). Apresentaremos um estudo mais analítico da conjectura motivados pelo teorema proposto por S. Hwang em sua tese de doutorado [32]:

Teorema 4.1. [32] Seja $\left(M^{3}, g, f\right)$ uma solução para (1.26). Se $|\nabla f|^{2}=g(\nabla f, \nabla f)$ é uma função apenas de $f$, então a métrica $g$ é Einstein.

Inspirados por este teorema, nos perguntamos se $g(\nabla f, \nabla f)$, numa variedade CPE $n$ dimensional, seria uma função apenas de $f$ de fato. Então, uma análise nos levou a uma expressão explícita para $|\nabla f|^{2}$ na métrica CPE.

No Teorema 4.5 mostraremos condições necessárias e suficientes para que a métrica CPE seja Einstein em qualquer dimensão.

\subsection{Preliminares}

Para provarmos o Teorema 4.5, precisamos de uma proposição, já demonstrada previamente em [35] (ver também [19]). Já que a demonstração desse resultado é curta, e fácil de acompanhar, vamos apresentá-la nesse trabalho.

Proposição 4.2 ([35], [19]). Seja $\left(M^{n}, g, f\right)$, uma métrica CPE com $f$ não-constante, e $B=\left\{x \in M^{n} \mid f(x)=-1\right\}$. Então $B$ tem medida n-dimensional nula. 
Demonstração. Seja $B^{\prime}$ o conjunto dos pontos críticos de $f$ em $B$. Então $B \backslash B^{\prime}$ é uma união de hipersuperfícies.

Agora, para $p \in B^{\prime}$, nós usamos (1.26) para obter

$$
\nabla^{2} f(\xi, \xi)=\frac{R}{n(n-1)} g(\xi, \xi)>0
$$

para qualquer vetor tangente não-nulo $\xi$ no espaço tangente $T_{p} M$ em $p$ (lembrando que $R$ é positivo por (1.27)). Portanto, $p$ é um ponto crítico não-degenerado de $f$. Mais ainda, $f$ tem seu mínimo local em $p$. Tais pontos críticos não-degenerados são isolados, e por isso $B^{\prime}$ tem que ser finito. Isso nos permite concluir que $B=B^{\prime} \bigcup\left(B \backslash B^{\prime}\right)$ tem medida $n$-dimensional nula, como queriamos demonstrar.

Observação 4.3. Precisamos fazer uma análise do conjunto

$$
M_{0}=\{x \in M ; 1+f(x)<0\}
$$

e supor regularidade, pelo menos, nas componentes conexas de sua fronteira (de tal maneira que possamos aplicar a fórmula de Stokes).

Seja $M_{0}^{\prime}$ uma componente conexa de $M_{0}$ e $\partial M_{0}^{\prime}$ a fronteira de $M_{0}^{\prime}$. Usaremos ao longo do texto que $\partial M_{0}^{\prime}$ é regular (i.e, satisfaz a fórmula de Stokes). Por definição, $f=-1$ em $\partial M_{0}^{\prime}$. Veja que tal fronteira é não-vazia, pois se o fosse $f$ teria sinal definido, isto é, $f>0$ ou $f<0$, e (pelo Lema 1 em [35]) a conjectura estaria demonstrada.

Mostraremos a seguir um lema (ver [19]) que nos permite definir o vetor normal na fronteira de $M_{0}$.

Lema 4.4 ([19]). Seja $\left(M^{n}, g, f\right)$ uma métrica CPE com $f$ não constante. Considerando o conjunto

$$
M_{0}=\{x \in M ; 1+f(x)<0\} .
$$

Então $\nabla f \neq 0$ para todo $p \in \partial M_{0}$.

Demonstração. Considere o subconjunto (4.1) onde $\partial M_{0} \subseteq f^{-1}(-1)$ é a fronteira desse conjunto. Seja $p \in f^{-1}(-1)$ tal que $(\nabla f)(p)=0$, isto é, $p$ é um ponto crítico de $f$, então pela equação (1.26) temos que

$$
\nabla^{2} f_{p}(X, X)=\frac{R}{n(n-1)} g_{p}(X, X)>0
$$


para qualquer vetor não-nulo $X \in T_{p} M$. Logo, $p \in f^{-1}(-1)$ é um ponto de mínimo não-degenerado. Sendo $M_{0}=\{x \in M ; 1+f(x)<0\}$ e sua fronteira $\partial M_{0} \subseteq f^{-1}(-1)$. Um ponto crítico de $f$ em $\partial M_{0}$ é de mínimo local pelo que acabamos de discutir acima. Portanto, é impossível que $\nabla f=0$ em algum ponto de $\partial M_{0}$. Suponha que $p \in \partial M_{0} \subseteq$ $f^{-1}(-1)$ tal que $(\nabla f)(p)=0$. Então existe $\varepsilon>0$ tal que $f(q) \geq f(p)$ para todo $q \in B(p, \varepsilon)$. Logo, $f(q)+1 \geq f(p)+1=0$ para todo $q \in B(p, \varepsilon)$. O que é um absurdo, pois já que $p \in \partial M_{0}$ para todo $\varepsilon>0$ existe $\bar{q} \in B(p, \varepsilon)$ tal que $1+f(\bar{q})<0$.

Agora estamos em condições de demonstrar os resultados principais.

\subsection{Resultados principais}

Teorema 4.5. Seja $\left(M^{n}, g, f\right)$ uma métrica CPE n-dimensional. Então $M^{n}$ é Einstein se, e somente se,

$$
|\nabla f|^{2}+\frac{R f^{2}}{n(n-1)}=\Lambda
$$

onde $\Lambda$ é uma constante real.

Demonstração. Primeiro, suponha que $\left(M^{n}, g, f\right)$ é uma métrica CPE. Suponha também que a métrica $g$ é Einstein. Nessas condições, podemos usar (1.26) para concluir que

$$
0=(1+f) \stackrel{\circ}{R}_{i j}=\nabla_{i} \nabla_{j} f+\frac{R f}{n(n-1)} g_{i j}
$$

Portanto, segue que

$$
\begin{aligned}
0 & =\nabla_{i} \nabla_{j} f \nabla^{j} f+\frac{R f}{n(n-1)} g_{i j} \nabla^{j} f \\
& =\frac{1}{2} \nabla_{i}|\nabla f|^{2}+\frac{R}{n(n-1)} f \nabla_{i} f \\
& =\frac{1}{2} \nabla_{i}\left(|\nabla f|^{2}+\frac{R f^{2}}{n(n-1)}\right)
\end{aligned}
$$

Então, por (4.3) temos (4.2).

Por outro lado, assumindo que $\left(M^{n}, g, f\right)$, satisfaz (4.2). Tomando sua derivada e usando 
(1.26) obtemos

$$
\begin{aligned}
0 & =\nabla_{i}|\nabla f|^{2}+\frac{2 R}{n(n-1)} f \nabla_{i} f \\
& =2 \nabla_{i} \nabla_{j} f \nabla^{j} f+\frac{2 R}{n(n-1)} f g_{i j} \nabla^{j} f=2(1+f) \stackrel{\circ}{R}_{i j} \nabla^{j} f
\end{aligned}
$$

Conseqüentemente, pela Proposição 4.2 e pela continuidade de $\stackrel{\circ}{R}_{i j}$ temos que $\stackrel{\circ}{R}_{i j} \nabla^{j} f$ se anula para todo $p \in M$. Logo, a equação acima implica que

$$
\operatorname{Ric}(\nabla f, .)=\frac{R}{n} g(\nabla f, .) .
$$

Agora, se derivarmos duas vezes (4.2) chegamos em

$$
0=\nabla_{i} \nabla_{k}|\nabla f|^{2}+\frac{2 R}{n(n-1)} \nabla_{i} f \nabla_{k} f+\frac{2 R f}{n(n-1)} \nabla_{i} \nabla_{k} f
$$

Contraindo (4.5) em $i$ e $k$ temos

$$
\begin{aligned}
0 & =g^{i k} \nabla_{i} \nabla_{k}|\nabla f|^{2}+\frac{2 R}{n(n-1)} g^{i k} \nabla_{i} f \nabla_{k} f+\frac{2 R f}{n(n-1)} g^{i k} \nabla_{i} \nabla_{k} f \\
& =\Delta|\nabla f|^{2}+\frac{2 R}{n(n-1)}|\nabla f|^{2}+\frac{2 R f \Delta f}{n(n-1)}
\end{aligned}
$$

Logo, segue de (1.27) que

$$
0=\Delta|\nabla f|^{2}+\frac{2 R}{n(n-1)}|\nabla f|^{2}-\frac{2 R^{2} f^{2}}{n(n-1)^{2}} .
$$

Lembrando que a fórmula de Böchner [9] é

$$
\frac{1}{2} \Delta|\nabla f|^{2}=\left|\nabla^{2} f\right|^{2}+\operatorname{Ric}(\nabla f, \nabla f)+g(\nabla f, \nabla(\Delta f)) .
$$

Substituindo (1.27) em (4.7) afirmamos que

$$
\Delta|\nabla f|^{2}=2\left|\nabla^{2} f\right|^{2}+2 \operatorname{Ric}(\nabla f, \nabla f)-2 \frac{R}{n-1}|\nabla f|^{2} .
$$

Agora, combine (4.6) com (4.8) para conseguir que

$$
0=2\left|\nabla^{2} f\right|^{2}+2 R i c(\nabla f, \nabla f)-\frac{2 R^{2} f^{2}}{n(n-1)^{2}}-\frac{2 R}{n}|\nabla f|^{2}
$$


Note que, por (1.26) e (1.27), temos

$$
\begin{aligned}
(1+f)^{2}\left|R_{i c}\right|^{2} & =(1+f)^{2} g^{i k} g^{j l} \stackrel{\circ}{R}_{i j} \stackrel{\circ}{R}_{k l} \\
& =g^{i k} g^{j l}\left(\nabla_{i} \nabla_{j} f+\frac{R f}{n(n-1)} g_{i j}\right)\left(\nabla_{k} \nabla_{l} f+\frac{R f}{n(n-1)} g_{k l}\right) \\
& =\left|\nabla^{2} f\right|^{2}-\frac{R^{2} f^{2}}{n(n-1)^{2}} .
\end{aligned}
$$

Além disso, comparando (4.10) com (4.9) concluímos que

$$
0=\left.2(1+f)^{2}\left|\operatorname{Ric}^{2}+2 \operatorname{Ric}(\nabla f, \nabla f)-\frac{2 R}{n}\right| \nabla f\right|^{2} .
$$

Então, por (4.4) vamos ver que

$$
\operatorname{Ric}(\nabla f, \nabla f)=\frac{R}{n} g(\nabla f, \nabla f)=\frac{R}{n}|\nabla f|^{2} .
$$

Enfim, (4.11) se reduz a

$$
0=2(1+f)^{2} \mid \text { Ric }^{2}
$$

E pela Proposição 4.2 conclui-se que Ric $=0 \mathrm{em} M^{n}$. Portanto a métrica $g$ é Einstein.

Corolário 4.6. Seja $\left(M^{n}, g, f\right)$ uma métrica CPE n-dimensional e $\nabla f$ autovetor de Ric, isto é, $R_{i j} \nabla^{j} f=\lambda \nabla_{i} f$, onde o autovalor associado $\lambda=\frac{R}{4}$. Então $M^{n}$ é uma variedade de Einstein.

Demonstração. Basta observar que, pela equação fundamental (1.26) e pela hipótese, temos

$$
0=(1+f)\left(\lambda-\frac{R}{4}\right)=(1+f) \stackrel{\circ}{R}_{i j} \nabla^{j} f=\frac{1}{2} \nabla_{i}\left(|\nabla f|^{2}+\frac{R f^{2}}{n(n-1)}\right) .
$$

E então usamos o Teorema 4.5 para concluirmos.

Pelas idéias do Teorema 4.5, vê-se que uma análise da função $G=|\nabla f|^{2}+\frac{R f^{2}}{n(n-1)}$ pode ser interessante para a conjectura (veja também [19]). Vamos, em seguida, mostrar um outro resultado que caminha nessa direção. Vamos demonstrar um lema técnico primeiramente (uma consequência do Teorema 4.5):

Lema 4.7. Seja $\left(M^{n}, g, f\right)$ uma métrica CPE. Defina em $M$ a função suave

$$
G=|\nabla f|^{2}+\frac{R f^{2}}{n(n-1)}
$$


Então,

$$
\frac{1}{2} \Delta G=(1+f)^{2}|\stackrel{\circ}{R} i c|^{2}+\stackrel{\circ}{R} i c(\nabla f, \nabla f)
$$

Demonstração. Tomando a derivada duas vezes da equação $G=|\nabla f|^{2}+\frac{R f^{2}}{n(n-1)}$ temos

$$
\nabla_{i} \nabla_{k} G=\nabla_{i} \nabla_{k}|\nabla f|^{2}+\frac{2 R}{n(n-1)} \nabla_{i} f \nabla_{k} f+\frac{2 R f}{n(n-1)} \nabla_{i} \nabla_{k} f
$$

Portanto, contraíndo (4.13) em $i$ e $k$ obtemos

$$
\begin{aligned}
g^{i k} \nabla_{i} \nabla_{k} G & =g^{i k} \nabla_{i} \nabla_{k}|\nabla f|^{2}+\frac{2 R}{n(n-1)} g^{i k} \nabla_{i} f \nabla_{k} f+\frac{2 R f}{n(n-1)} g^{i k} \nabla_{i} \nabla_{k} f \\
& =\Delta|\nabla f|^{2}+\frac{2 R}{n(n-1)}|\nabla f|^{2}+\frac{2 R f \Delta f}{n(n-1)} .
\end{aligned}
$$

Segue de $\Delta f=-\frac{R f}{n-1}$ que

$$
\Delta G=\Delta|\nabla f|^{2}+\frac{2 R}{n(n-1)}|\nabla f|^{2}-\frac{2 R^{2} f^{2}}{n(n-1)^{2}}
$$

Lembrando a fórmula de Bochner:

$$
\frac{1}{2} \Delta|\nabla f|^{2}=\left|\nabla^{2} f\right|^{2}+\operatorname{Ric}(\nabla f, \nabla f)+g(\nabla f, \nabla(\Delta f))
$$

Substituindo $\Delta f=-\frac{R f}{n-1}$ em (4.15) obtemos

$$
\Delta|\nabla f|^{2}=2\left|\nabla^{2} f\right|^{2}+2 \operatorname{Ric}(\nabla f, \nabla f)-2 \frac{R}{n-1}|\nabla f|^{2} .
$$

Combinando (4.14) com (4.16) temos

$$
\Delta G=2\left|\nabla^{2} f\right|^{2}+2 R i c(\nabla f, \nabla f)-\frac{2 R^{2} f^{2}}{n(n-1)^{2}}-\frac{2 R}{n}|\nabla f|^{2} .
$$

Lembrando que

$$
\begin{aligned}
(1+f)^{2} \mid \text { Ric }^{2} & =(1+f)^{2} g^{i k} g^{j l} \stackrel{\stackrel{R}{R}}{i j}^{R_{k l}} \\
& =g^{i k} g^{j l}\left(\nabla_{i} \nabla_{j} f+\frac{R f}{n(n-1)} g_{i j}\right)\left(\nabla_{k} \nabla_{l} f+\frac{R f}{n(n-1)} g_{k l}\right) \\
& =\left|\nabla^{2} f\right|^{2}-\frac{R^{2} f^{2}}{n(n-1)^{2}} .
\end{aligned}
$$


Então,

$$
2(1+f)^{2}\left|R_{i c}^{\circ}\right|^{2}+2 \frac{R^{2} f^{2}}{n(n-1)^{2}}=2\left|\nabla^{2} f\right|^{2} .
$$

Logo, comparando (4.19) com (4.17) obtemos o resultado.

Uma consequência imediata desse lema é:

Corolário 4.8. Seja $\left(M^{n}, g, f\right)$ uma métrica $C P E$. Se $\stackrel{\circ}{R} i c \geq 0$ então $M$ é uma variedade de Einstein.

Demonstração. De fato, pelo Lema (4.7) temos que

$$
\frac{1}{2} \int_{M} \Delta G=\int_{M}(1+f)^{2}|\stackrel{\circ}{R} i c|^{2}+\int_{M} \stackrel{\circ}{R} i c(\nabla f, \nabla f)
$$

isto é, por hipótese e usando a fórmula de Stokes

$$
0=\int_{M}(1+f)^{2}|\stackrel{\circ}{R} i c|^{2} \geq 0
$$

Então, pela Proposição 4.2 temos que $\stackrel{\circ}{\text { Ric }}=0$, como queríamos.

A seguir, mostraremos outro resultado que segue também como conseqüência do Lema 4.7.

Teorema 4.9. Seja $\left(M^{n}, g, f\right)$ uma métrica CPE, G definido por (4.12) e $M_{0}^{\prime}$ definido como na Observação 4.3. Suponha

$$
\Delta G=0 \quad \text { em } \quad \partial M_{0}^{\prime}
$$

Então g é Einstein.

Demonstração. Como $\partial M_{0}^{\prime}$ é uma componente conexa de $\partial M_{0} \subseteq f^{-1}(-1)$, então pelo lema 4.7 e pela hipótese temos que

$$
\stackrel{\circ}{\operatorname{Ric}}(\nabla f, \nabla f)=0
$$

em $\partial M_{0}^{\prime}$. Usando a fórmula de Stokes, segunda identidade de Bianchi contraída, Lema 
4.4 e 4.20 temos que

$$
\begin{aligned}
\int_{M_{0}^{\prime}}(1+f)\left|\stackrel{\circ}{R}_{i j}\right|^{2} & =\int_{M_{0}^{\prime}} g^{i k} g^{j l}\left(\nabla_{i} \nabla_{j} f+\frac{R f}{n(n-1)} g_{i j}\right) \stackrel{\circ}{R}_{k l} \\
& =\int_{M_{0}^{\prime}} g^{i k} g^{j l} \nabla_{i} \nabla_{j} f \stackrel{\circ}{R}_{k l} \\
& =-\int_{M_{0}^{\prime}} g^{i k} g^{j l} \nabla_{j} f \nabla_{i} \stackrel{\circ}{R}_{k l}+\int_{\partial M_{0}^{\prime}} g^{i k} g^{j l} \nabla_{j} f \stackrel{\circ}{R}_{k l}\left(-\frac{\nabla_{i} f}{|\nabla f|}\right) \\
& =-\int_{\partial M_{0}^{\prime}} \frac{1}{|\nabla f|} \stackrel{\circ}{R}_{k l} \nabla^{k} f \nabla^{l} f=-\int_{\partial M_{0}^{\prime}} \frac{1}{|\nabla f|} \stackrel{\circ}{R} i c(\nabla f, \nabla f)=0 .
\end{aligned}
$$

Logo,

$$
\int_{M_{0}^{\prime}}(1+f)\left|\stackrel{\circ}{R}_{i j}\right|^{2}=0
$$

Já que $0 \geq(1+f)\left|\stackrel{\circ}{R}_{i j}\right|^{2}$ em $M_{0}^{\prime}$ temos que $(1+f)\left|\stackrel{\circ}{R}_{i j}\right|^{2}=0$, por continuidade e (4.21). Logo, pela Proposição 4.2 nós temos $\stackrel{\circ}{\text { Ric }}=0$ em $M_{0}^{\prime}$. Isto é, $\stackrel{\circ}{\text { Ric }}=0$ in $M_{0}$.

Portanto, pelo lema 1 em [35]

$$
0=\int_{M}(1+f)|\stackrel{\circ}{R} i c|^{2}=\int_{M_{0}^{c}}(1+f)|\stackrel{\circ}{R} i c|^{2}+\int_{M_{0}}(1+f)|\stackrel{\circ}{R} i c|^{2} .
$$

Então a equação (4.21) garante que

$$
0=\int_{M}(1+f)|\stackrel{\circ}{R} i c|^{2}=\int_{M_{0}^{c}}(1+f)|\stackrel{\circ}{R} i c|^{2} .
$$

Mas já que $M_{0}^{c}=\{x \in M ; 0 \leq(1+f)(x)\}$ temos que $0 \leq(1+f)|\stackrel{\circ}{R} i c|^{2}$ em $M_{0}^{c}$, por continuidade e (4.22), obtemos $(1+f) \stackrel{\circ}{R} i c=0$ em $M_{0}^{c}$, isto é, pela proposição (4.2) $\stackrel{\circ}{R} i c=0$ em $M_{0}^{c}$. E finalmente temos que $\stackrel{\circ}{R} i c=0$ em $M$, ou seja $g$ é Einstein.

Observação 4.10. Como a função $G$ é contínua, então ela atinge seu máximo (mínimo) em $M$. Pelo princípio do máximo forte, se $\Delta G \geq(\leq) 0$ então $G$ é constante em $M$ (sobre discussão sobre princípio do máximo veja [19]).

Os resultados desse capítulo podem ser encontrados em [44]. 


\section{Capítulo 5}

\section{Métrica CPE em dimensão $n=4$}

Nesse capítulo, vamos estudar a métrica CPE em variedades de dimensão 4 (veja seções 1.1, 1.6 e 1.7). Inspirados pelo desenvolvimento histórico do estudo da conjectura CPE com as condições localmente conformemente plana (ver [41]), semi conformemente plano (ou, (anti) self dual tensor de Weyl) (ver [4]) e tensor curvatura harmônico (veja [19]) nós vamos observar o comportamento da métrica CPE sob a condição $\operatorname{div}\left(W^{+}\right)=0$. É claro que se trocarmos a condição $\delta W^{+}=0$ pela condição $\delta W^{-}=0$ as conclusões serão exatamente as mesmas. Ao longo do texto, usaremos as seguintes notações para indicar o divergente. Veja como exemplo o divergente do tensor de Weyl:

$$
\delta W=-\nabla^{i} W_{i j k l}=-g^{i s} \nabla_{s} W_{i j k l} .
$$

\subsection{Preliminares}

Primeiro, mostraremos alguns resultados essenciais para o desenvolvimento desse capítulo.

Lema 5.1. [4] Seja $\left(M^{n}, g, f\right)$ uma métrica CPE. Então:

$$
\begin{aligned}
(1+f)\left(\nabla_{j} R_{i k}-\nabla_{i} R_{j k}\right) & =-R_{i j k s} \nabla^{s} f-\left(\nabla_{j} f R_{i k}-\nabla_{i} f R_{j k}\right) \\
& +\frac{R}{n-1}\left(\nabla_{j} f g_{i k}-\nabla_{i} f g_{j k}\right)
\end{aligned}
$$

Demonstração. Como $M^{n}$ tem curvatura escalar constante e $g$ é paralelo, podemos derivar a equação (1.26) para obtermos

$$
\nabla_{j} R_{i k}=\nabla_{j} \nabla_{i} \nabla_{k} f-\left(\nabla_{j} f\right) R_{i k}-f \nabla_{j} R_{i k}+\frac{R}{n-1} \nabla_{j} f g_{i k}
$$


dando-nos

$$
(1+f) \nabla_{j} R_{i k}=\nabla_{j} \nabla_{i} \nabla_{k} f-\left(\nabla_{j} f\right) R_{i k}+\frac{R}{n-1} \nabla_{j} f g_{i k}
$$

e, arranjando os índices,

$$
(1+f) \nabla_{i} R_{j k}=\nabla_{i} \nabla_{j} \nabla_{k} f-\left(\nabla_{i} f\right) R_{j k}+\frac{R}{n-1} \nabla_{i} f g_{j k}
$$

Se usarmos (1.9) e subtrairmos as duas equações acima, obteremos o resultado.

Lembre-se que o tensor de Weyl, para $n \geq 3$, é dado pela expressão (1.8) e o tensor de Cotton é dado por (1.11). Mas como na métrica CPE temos que a curvatura escalar é constante, isso nos dá que

$$
C_{i j k}=\nabla_{i} R_{j k}-\nabla_{j} R_{i k}
$$

Agora estamos aptos a demonstrar a relação entre os tensores de Weyl e de Cotton na métrica CPE. Precisamente, temos o seguinte lema.

Lema 5.2. [4] Seja $\left(M^{n}, g, f\right)$ uma métrica CPE. Então:

$$
\begin{aligned}
(1+f) C_{i j k}=W_{i j k s} \nabla^{s} f & -\frac{R}{n-2}\left(\nabla_{j} f g_{i k}-\nabla_{i} f g_{j k}\right)+\frac{n-1}{n-2}\left(\nabla_{j} f R_{i k}-\nabla_{i} f R_{j k}\right) \\
& -\frac{1}{n-2}\left(R_{i s} \nabla^{s} f g_{j k}-R_{j s} \nabla^{s} f g_{i k}\right)
\end{aligned}
$$

Demonstração. Primeiramente, podemos comparar (5.1) com (5.2) e obter

$$
(1+f) C_{i j k}=R_{i j k s} \nabla^{s} f+\left(\nabla_{j} f R_{i k}-\nabla_{i} f R_{j k}\right)-\frac{R}{n-1}\left(\nabla_{j} f g_{i k}-\nabla_{i} f g_{j k}\right) .
$$

Por outro lado, segue de (1.8) que

$$
\begin{aligned}
R_{i j k s} \nabla^{s} f=W_{i j k s} \nabla^{s} f & +\frac{1}{n-2}\left(R_{i k} \nabla_{j} f-R_{j k} \nabla_{i} f\right)-\frac{1}{n-2}\left(R_{i s} \nabla^{s} f g_{j k}-R_{j s} \nabla^{s} f g_{i k}\right) \\
& -\frac{R}{(n-1)(n-2)}\left(\nabla_{j} f g_{i k}-\nabla_{i} f g_{j k}\right) .
\end{aligned}
$$

Se combinarmos (5.4) com (5.5) teremos o resultado. 
A seguir, vamos definir o tensor $T$, introduzido por [14] (veja também [4]), como

$$
\begin{aligned}
T_{i j k} & =\frac{(n-1)}{(n-2)}\left(R_{i k} \nabla_{j} f-R_{j k} \nabla_{i} f\right)-\frac{1}{(n-2)}\left(R_{i s} \nabla^{s} f g_{j k}-R_{j s} \nabla^{s} f g_{i k}\right) \\
& -\frac{R}{(n-2)}\left(\nabla_{j} f g_{i k}-\nabla_{i} f g_{j k}\right) .
\end{aligned}
$$

Tendo essa definição em mente, podemos deduzir de (5.3) que

$$
(f+1) C_{i j k}=W_{i j k s} \nabla^{s} f+T_{i j k}
$$

Enunciaremos alguns resultados sobre métricas CPE, encontrados na literatura:

Proposição 5.3. [19] O conjunto crit $(f)=\{x \in M ; \nabla f=0\}$ tem medida nula.

Teorema 5.4. [19] A conjectura CPE é verdadeira se o tensor curvatura for harmônico, isto é, se o divergente do tensor curvatura for identicamente nulo $\left(\nabla^{i} R_{i j k l}=0\right)$.

Observação 5.5. A condição $\nabla^{i} R_{i j k l}=0$ mencionada no teorema 5.4 acima é equivalente ao tensor de Ricci ser do tipo Codazzi, isto é, $\nabla_{i} R_{j k}-\nabla_{j} R_{i k}=0$. Nosso objetivo, a seguir, é demonstrar que $\operatorname{div}\left(W^{+}\right)=0$ implica no tensor de Ricci, $R_{i j}$, ser tipo Codazzi e então aplicar o Teorema 5.4.

\section{$5.2 \quad$ Resultados principais}

Para começarmos, precisamos mostrar o que significa de fato $\delta W^{+}$(divergente de $W^{+}$) numa métrica CPE 4-dimensional. Veja que uma conseqüência imediata de (1.25) e de (1.12) é que

$$
\begin{aligned}
4 \delta W_{j k l}^{+} & =2 \nabla^{i}\left(W_{i j k l}+W_{i j \bar{k} \bar{l}}\right) \\
& =\left(C_{k l j}+C_{\bar{k} \bar{l} j}\right)
\end{aligned}
$$

Combinando (5.7) com (5.8) temos

$$
\begin{aligned}
4(1+f) \delta W_{j k l}^{+} & =(1+f)\left(C_{k l j}+C_{\bar{k} \bar{l} j}\right) \\
& =\left[W_{k l j s} \nabla^{s} f+W_{\bar{k} \bar{l} j s} \nabla^{s} f+T_{l k j}+T_{\bar{l} \bar{k} j}\right]
\end{aligned}
$$


Agora, usando que $\delta W^{+}=0$, temos que

$$
-W_{k l j s} \nabla^{s} f-W_{\bar{k} \bar{l} j s} \nabla^{s} f=T_{k l j}+T_{\bar{k} \bar{l} j} .
$$

Logo,

$$
0=-\left(W_{k l j s} \nabla^{s} f+W_{\bar{k} \bar{l} j s} \nabla^{s} f\right) \nabla^{j} f=\left(T_{k l j}+T_{\bar{k} \bar{l} j}\right) \nabla^{j} f
$$

E então, renomeando os índices,

$$
\left(T_{i j k}+T_{\bar{i} \bar{j} k}\right) \nabla^{k} f=0
$$

Por outro lado, a equação (5.6) nos permite deduzir que

$$
\begin{aligned}
T_{i j k} \nabla^{k} f= & \frac{3}{2}\left(R_{i k} \nabla_{j} f-R_{j k} \nabla_{i} f\right) \nabla^{k} f-\frac{1}{2}\left(R_{i s} \nabla^{s} f g_{j k}-R_{j s} \nabla^{s} f g_{i k}\right) \nabla^{k} f \\
& -\frac{R}{2}\left(\nabla_{j} f g_{i k}-\nabla_{i} f g_{j k}\right) \nabla^{k} f \\
= & \left(R_{i k} \nabla_{j} f-R_{j k} \nabla_{i} f\right) \nabla^{k} f .
\end{aligned}
$$

Então, de (5.10) segue que

$$
\left(R_{i k} \nabla_{j} f-R_{j k} \nabla_{i} f\right) \nabla^{k} f+\left(R_{\bar{i} k} \nabla_{\bar{j}} f-R_{\bar{j} k} \nabla_{\bar{i}} f\right) \nabla^{k} f=0 .
$$

Considere uma base ortonormal $\left\{e_{1}, e_{2}, e_{3}, e_{4}\right\}$, diagonalizando Ric no ponto $q$, tal que $\nabla f(q) \neq 0$, isto é, $\operatorname{Ric}_{q}\left(e_{i}, e_{j}\right)=\lambda_{i}(q) \delta_{i j}$, com autovalores associados $\lambda_{k},(k=1, \ldots, 4)$, respectivamente. É importante esclarecer que o conjunto de pontos regulares de $M^{4}$, denotado por $\left\{p \in M^{n}: \nabla f(p) \neq 0\right\}$, é denso em $M^{4}$, por (5.3). Do contrário, $f$ seria constante em um aberto de $M^{4}$; na verdade, por (1.26), essa constante seria igual a zero, mas pela teoria dos conjuntos nodais $f$ não pode ser identicamente nula num aberto de $M^{4}$, veja [21]. Para essa discussão sobre a densidade do conjunto $\left\{p \in M^{n}: \nabla f(p) \neq 0\right\}$ recomendamos também a Proposição 2.2 em [19]. De agora em diante, a menos que seja mencionado o contrário, vamos voltar nossas atenções aos pontos regulares.

Lema 5.6. Seja $\left(M^{4}, g, f\right)$ uma métrica CPE satisfazendo (1.26) tal que $\delta W^{+}=0$. Então $\nabla f$ é um autovetor do tensor de Ricci.

Demonstração. Note que, novamente escolhendo um referencial ortonormal que diago- 
naliza o tensor de Ricci e substituindo $R_{i j}=\lambda_{i} \delta_{i j}$ em (5.12) temos

$$
\begin{aligned}
0 & =\left(R_{i k} \nabla_{j} f-R_{j k} \nabla_{i} f\right) \nabla^{k} f+\left(R_{\bar{i} k} \nabla_{\bar{j}} f-R_{\bar{j} k} \nabla_{\bar{i}} f\right) \nabla^{k} f \\
& =\left(\lambda_{i} \delta_{i k} \nabla_{j} f-\lambda_{j} \delta_{j k} \nabla_{i} f\right) \nabla^{k} f+\left(\lambda_{\bar{i}} \delta_{\bar{i} k} \nabla_{\bar{j}} f-\lambda_{\bar{j}} \delta_{\bar{j} k} \nabla_{\bar{i}} f\right) \nabla^{k} f \\
& =\left(\lambda_{i}-\lambda_{j}\right) \nabla_{i} f \nabla_{j} f+\left(\lambda_{\bar{i}}-\lambda_{\bar{j}}\right) \nabla_{\bar{i}} f \nabla_{\bar{j}} f
\end{aligned}
$$

ou seja, por (1.19),

$$
\left\{\begin{array}{l}
\left(\lambda_{1}-\lambda_{2}\right) \nabla_{1} f \nabla_{2} f+\left(\lambda_{3}-\lambda_{4}\right) \nabla_{3} f \nabla_{4} f=0 \\
\left(\lambda_{1}-\lambda_{3}\right) \nabla_{1} f \nabla_{3} f+\left(\lambda_{4}-\lambda_{2}\right) \nabla_{4} f \nabla_{2} f=0 \\
\left(\lambda_{1}-\lambda_{4}\right) \nabla_{1} f \nabla_{4} f+\left(\lambda_{2}-\lambda_{3}\right) \nabla_{2} f \nabla_{3} f=0
\end{array}\right.
$$

Nós vamos mostrar que $\nabla f$, sempre que não-nulo, é um autovetor de Ric. Na verdade, levando em consideração que $\nabla f(p) \neq 0$ temos que, pelo menos, uma das componentes coordenadas do gradiente da função $f$ é diferente de zero, i.e, $\left(\nabla_{j} f\right)(p) \neq 0,1 \leq j \leq 4$. Se isso ocorre para exatamente um índice, então $\nabla f=\left(\nabla_{j} f\right) e_{j}$ para algum $j$, nos dando que $\operatorname{Ric}(\nabla f)=\lambda_{j} \nabla f$. Por outro lado, se nós temos que $\left(\nabla_{j} f\right)(p) \neq 0$ para duas componentes distintas, sem perda de generalidade podemos supor $\nabla_{1} f \neq 0, \nabla_{2} f \neq 0$, $\nabla_{3} f=0$ e $\nabla_{4} f=0$. Então, por (5.13) nós teremos que $\lambda_{1}=\lambda_{2}$. Nesse caso teremos que $\nabla f=\left(\nabla_{1} f\right) e_{1}+\left(\nabla_{2} f\right) e_{2}$. E desse fato, podemos ver que

$$
\begin{aligned}
\operatorname{Ric}(\nabla f) & =\operatorname{Ric}\left(\left(\nabla_{1} f\right) e_{1}+\left(\nabla_{2} f\right) e_{2}\right)=\left(\nabla_{1} f\right) \operatorname{Ric}\left(e_{1}\right)+\left(\nabla_{2} f\right) \operatorname{Ric}\left(e_{2}\right) \\
& =\left(\nabla_{1} f\right) \lambda_{1} e_{1}+\left(\nabla_{2} f\right) \lambda_{2} e_{2}=\lambda \nabla f .
\end{aligned}
$$

O próximo caso, isto é, $\left(\nabla_{j} f\right)(p) \neq 0$ para três componentes coordenadas do gradiente da função $f$ é análogo ao caso anterior. Agora, nos resta analisar o caso que $\left(\nabla_{j} f\right)(p) \neq 0$ para $j=1,2,3,4$. Para esse caso, vamos usar o sistema (5.13) mais uma vez. Se tomarmos o quadrado de cada equação do sistema e depois somarmos os resultados, obtemos

$$
\begin{aligned}
& \left(\lambda_{1}-\lambda_{2}\right)^{2}\left(\nabla_{1} f \nabla_{2} f\right)^{2}+\left(\lambda_{3}-\lambda_{4}\right)^{2}\left(\nabla_{3} f \nabla_{4} f\right)^{2} \\
+ & \left(\lambda_{1}-\lambda_{3}\right)^{2}\left(\nabla_{1} f \nabla_{3} f\right)^{2}+\left(\lambda_{4}-\lambda_{2}\right)^{2}\left(\nabla_{4} f \nabla_{2} f\right)^{2} \\
+ & \left(\lambda_{1}-\lambda_{4}\right)^{2}\left(\nabla_{1} f \nabla_{4} f\right)^{2}+\left(\lambda_{2}-\lambda_{3}\right)^{2}\left(\nabla_{2} f \nabla_{3} f\right)^{2}=0 .
\end{aligned}
$$

Portanto, $\lambda_{1}=\lambda_{2}=\lambda_{3}=\lambda_{4}$. Donde segue que $\nabla f$ é um autovetor para Ric.

Observação 5.7. Observe que se $\nabla_{i} f \neq 0$ para todo $i \in\{1,2,3,4\}$, então, $\lambda_{1}=\ldots=\lambda_{4}$ pelo Lema 5.6. Como $R=\sum_{i=1}^{4} \lambda_{i}$ temos que $\frac{R}{4}$ é o autovalor de $\nabla f$. Portanto, podemos ver pelo Corolário 4.6 que a métrica é Einstein. Entretanto, se houver $\left(\nabla_{i} f\right)(p)=0$, 
para algum $i \in\{1,2,3,4\}$, em algum aberto de $M$ então o autovalor associado a $\nabla f$ não é necessariamente constante.

Os conjuntos de nível da função potencial $f$ nas métricas tipo solitons são muito importantes. Por esse motivo, vamos fazer uma análise desses conjuntos na métrica CPE. Seja $f: M \rightarrow \mathbb{R}$ uma função diferenciável, onde M é uma variedade diferenciável de dimensão $n=4$ compacta. Suponha que, $f$ não seja constante. Note que, $f$ possui, pelo menos, duas singularidades, o máximo e o mínimo de $f$. Logo, $f$ é uma submersão em $M \backslash \operatorname{crit}(f)$, onde $\operatorname{crit}(f)=\{p \in M ; \nabla f(p)=0\}$, e portanto podemos estudar a folheação definida por $f^{-1}$ em $M \backslash c r i t(f)$. Note que, as cartas locais definem uma estrutura de variedade folheada, onde as folhas são as componentes conexas dos conjuntos de nível $f^{-1}(c)=\left\{p \in M^{n}: f(p)=c\right\}$. Mas temos que na métrica CPE, o conjunto crit $(f)$ tem medida $n$-dimensional nula (veja [19] e [4] e Proposição 5.3). Com isso, temos uma folheação para variedade CPE, $M \backslash C r i t(f)$, onde as folhas são componentes conexas dos conjuntos de nível. Seja $\Sigma_{c}=f^{-1}(c)=\left\{p \in M^{4} ; f(p)=c\right\}$, onde $c \neq-1$. Defina um referencial ortonormal $\left\{e_{1}, e_{2}, e_{3}, e_{4}\right\}$ que diagonaliza o tensor de Ricci em um ponto regular de $M^{4}$ tal que $e_{4}=N=\frac{\nabla f}{|\nabla f|}$ em $\Sigma_{c}$.

Proposição 5.8. Considere $\left(M^{4}, g, f\right)$ uma métrica CPE satisfazendo (1.26) tal que $\delta W^{+}=0$. Seja $c \neq-1$ um valor regular de $f$ e $\Sigma_{c}=\left\{p \in M^{4} ; f(p)=c\right\}$ um conjunto de nivel de $f$. Tome $N=e_{4}=\frac{\nabla f}{|\nabla f|}$ e escolha um referencial ortonormal $\left\{e_{1}, e_{2}, e_{3}\right\}$ tangente a $\Sigma_{c}$, que diagonaliza o tensor de Ricci, isto é, $R_{i j}=\lambda_{i} \delta_{i j}$ para $1 \leq i, j \leq 4$. Nessas condiçôes, temos as seguintes propriedades:

1. $R_{4 a}=0$, e portanto $\stackrel{\circ}{R}_{4 a}=0$, para qualquer $1 \leq a \leq 3$, em $\Sigma_{c}$.

2. $|\nabla f|^{2}$ é constante em $\Sigma_{c}$. Conseqüentemente, $|\nabla f|^{2}+\frac{R f^{2}}{n(n-1)}$ é constante em $\Sigma_{c}$ (ver Teorema 4.5).

3. A segunda forma fundamental e a curvatura média em $\Sigma_{c}$ são dadas por $h_{a b}=\frac{1}{|\nabla f|}\left[\frac{R}{4}+\frac{R f}{3}-(1+f) \lambda_{a}\right] g_{a b}$ e $H_{\Sigma_{c}}=\frac{1}{|\nabla f|}\left[(1+f) \lambda_{4}-\frac{R}{4}\right]$, respectivamente.

4. A segunda forma fundamental e a curvatura média se relacionam através do tensor $T$ (5.6) da seguinte forma

$$
h_{a b}-\frac{H_{\Sigma_{c}}}{3} g_{a b}=\frac{2(1+f)}{3|\nabla f|^{2}} T_{4 a a} g_{a b}
$$

Como conseqüência, temos que

$$
|\nabla f|^{2}\left|h_{a b}-\frac{H_{\Sigma_{c}}}{3} g_{a b}\right|^{2}=(1+f)^{2}\left(|\stackrel{\circ}{\operatorname{R} i c}|^{2}-\frac{4}{3} \stackrel{\circ}{R} i c(N, N)^{2}\right) \text {. }
$$




\section{Demonstração.}

1. Por hipótese, temos que $\delta W^{+}=0$ logo podemos usar o Lema (5.6), i.e, temos que $\operatorname{Ric}(\nabla f)=\lambda \nabla f$. Imediatamente, vemos que $g\left(\operatorname{Ric}(\nabla f), e_{a}\right)=\lambda g\left(\nabla f, e_{a}\right)=0$. Conseqüentemente,

$$
\stackrel{\circ}{\operatorname{Ric}}(Y, \nabla f)=0
$$

para todo campo $Y \in T_{p} \Sigma_{c}$. De fato, já que $\delta W^{+}=0$ implica em $\operatorname{Ric}(\nabla f)=\lambda \nabla f$ para um campo arbitrário $Y \in T_{p} \Sigma_{c}$ temos que

$$
\stackrel{\circ}{\operatorname{Ric}}(Y, \nabla f)=\left(\lambda-\frac{R}{n}\right) g(Y, \nabla f)=0 .
$$

2. O segundo item, segue diretamente do primeiro. Repare que por (5.14) temos, pela equação (1.26),

$$
\nabla_{a}|\nabla f|^{2}=(1+f) \stackrel{\circ}{\operatorname{Ric}}\left(\nabla f, e_{a}\right)-\frac{R f}{12} g\left(\nabla f, e_{a}\right)=0 .
$$

3. Por outro lado, já que $g\left(e_{a}, N\right)=0$, para $a \in\{1,2,3\}$, implica que

$$
e_{b} g\left(e_{a}, \nabla f\right)=g\left(\nabla_{e_{b}} e_{a}, \nabla f\right)+g\left(e_{a}, \nabla_{e_{b}} \nabla f\right)=0
$$

isto é,

$$
g\left(\nabla_{e_{b}} e_{a}, \frac{\nabla f}{|\nabla f|}\right)=-\frac{1}{|\nabla f|} g\left(e_{a}, \nabla_{e_{b}} \nabla f\right)
$$

Teremos pela equação fundamental da métrica CPE que a segunda forma fundamental é dada por

$$
\begin{aligned}
h_{a b} & =g\left(\nabla_{a} e_{b}, N\right)=\frac{1}{|\nabla f|} g\left(\nabla_{a} e_{b}, \nabla f\right)=-\frac{1}{|\nabla f|} g\left(e_{b}, \nabla_{a} \nabla f\right)=-\frac{\nabla_{a} \nabla_{b} f}{|\nabla f|} \\
& =\frac{1}{|\nabla f|}\left[\frac{R f}{12} g_{a b}-(1+f)\left(R_{a b}-\frac{R}{4} g_{a b}\right)\right] \\
& =\frac{1}{|\nabla f|}\left[\frac{R f}{12}-(1+f)\left(\lambda_{a}-\frac{R}{4}\right)\right] g_{a b}
\end{aligned}
$$

onde $a, b=\{1,2,3\}$ e, pela base que diagonaliza o tensor de Ricci, $\operatorname{Ric}\left(e_{a}, e_{b}\right)=$ $\lambda_{a} \delta_{a b}$. 
Se tomarmos o traço em (5.16) veremos que

$$
\begin{aligned}
H_{\Sigma_{c}}=\sum_{a=1}^{3} h_{a a} & =\frac{1}{|\nabla f|}\left[\frac{R f}{12}-(1+f)\left(\lambda_{a}-\frac{R}{4}\right)\right] g_{a a} \\
& =\frac{1}{|\nabla f|}\left[\frac{3 R f}{12}-(1+f)\left(\sum_{a=1}^{3} \lambda_{a}-\frac{3 R}{4}\right)\right] \\
& =\frac{1}{|\nabla f|}\left[\frac{3 R f}{12}-(1+f)\left[\left(R-\lambda_{4}\right)-\frac{3 R}{4}\right]\right] \\
& =\frac{1}{|\nabla f|}\left[\frac{3 R f}{12}-(1+f)\left(\frac{R}{4}-\lambda_{4}\right)\right] \\
& =\frac{1}{|\nabla f|}\left[(1+f) \lambda_{4}-\frac{R}{4}\right]
\end{aligned}
$$

4. Pelo terceiro item,

$$
\begin{aligned}
h_{a b}-\frac{H_{\Sigma_{c}}}{3} g_{a b} & =\frac{1}{|\nabla f|}\left[\frac{R}{4}+\frac{R f}{3}-(1+f) \lambda_{a}\right] g_{a b}-\frac{1}{3|\nabla f|}\left[(1+f) \lambda_{4}-\frac{R}{4}\right] g_{a b} \\
& =\frac{(1+f)}{3|\nabla f|}\left(R-\lambda_{4}-3 \lambda_{a}\right) g_{a b} \\
& =\frac{(1+f)}{3|\nabla f|}\left(R g_{a b}-R_{44} g_{a b}-3 R_{a b}\right) .
\end{aligned}
$$

Por outro lado, o tensor $T$ (5.6) nos dá que

$$
\begin{aligned}
T_{i j k} \nabla^{i} f & =\frac{3}{2}\left(\lambda_{k} \nabla_{k} f \nabla_{j} f-R_{j k}|\nabla f|^{2}\right)-\frac{1}{2}\left(\lambda_{i}|\nabla f|^{2}-\lambda_{j} \nabla_{j} f \nabla_{k} f\right) \\
& -\frac{R}{2}\left(\nabla_{j} f \nabla_{k} f-g_{j k}|\nabla f|^{2}\right) .
\end{aligned}
$$

Ou seja,

$$
T_{4 a a}=\frac{|\nabla f|}{2}\left(R-\lambda_{4}-3 \lambda_{a}\right)
$$

Com essa equação acima, concluímos a primeira parte do quarto item. Agora, por 
(5.17) temos que

$$
\begin{aligned}
\left|h_{a b}-\frac{H_{\Sigma_{c}}}{3} g_{a b}\right|^{2} & =\left|\frac{(1+f)}{3|\nabla f|}\left(R g_{a b}-R_{44} g_{a b}-3 R_{a b}\right)\right|^{2} \\
& =\frac{(1+f)^{2}}{9|\nabla f|^{2}}\left(3 R^{2}+3 R_{44}^{2}+9\left|R_{a b}\right|^{2}-6 R R_{a b} g^{a b}\right. \\
& \left.-6 R R_{44}+6 R_{44} R_{a b} g^{a b}\right) \\
& =\frac{(1+f)^{2}}{9|\nabla f|^{2}}\left(3 R^{2}+3 R_{44}^{2}+9\left|R_{a b}\right|^{2}-6 R\left(R-R_{44}\right)\right. \\
& \left.-6 R R_{44}+6 R_{44}\left(R-R_{44}\right)\right) \\
& =\frac{(1+f)^{2}}{9|\nabla f|^{2}}\left(9\left|R_{a b}\right|^{2}-3\left(R-R_{44}\right)^{2}\right) .
\end{aligned}
$$

Por outro lado, $|R i c|^{2}=\left|R_{a b}\right|^{2}+2 \sum_{a=1}^{3} R_{4 a}^{2}+R_{44}^{2}$. Lembre-se que $\nabla f$ é autovetor do tensor de Ricci, por isso temos que $R_{4 a}=0$. Portanto,

$$
\begin{aligned}
\left|h_{a b}-\frac{H_{\Sigma_{c}}}{3} g_{a b}\right|^{2} & =\frac{(1+f)^{2}}{|\nabla f|^{2}}\left(|R i c|^{2}-R_{44}^{2}\right)-\frac{(1+f)^{2}}{3|\nabla f|^{2}}\left(R-R_{44}\right)^{2} \\
& =\frac{(1+f)^{2}}{|\nabla f|^{2}}\left(|R i c|^{2}-\frac{4}{3} R_{44}^{2}-\frac{R^{2}}{3}+\frac{2 R R_{44}}{3}\right) \\
& =\frac{(1+f)^{2}}{|\nabla f|^{2}}\left(|\stackrel{R}{R} i c|^{2}-\frac{4}{3} R_{44}^{2}-\frac{R^{2}}{12}+\frac{2 R R_{44}}{3}\right) \\
& =\frac{(1+f)^{2}}{|\nabla f|^{2}}\left[|\stackrel{\circ}{R} i c|^{2}-\frac{\left(4 R_{44}-R\right)}{12}\right] .
\end{aligned}
$$

Como $\stackrel{\circ}{R}_{44}=\stackrel{\circ}{\operatorname{Ric}}(N, N)=R_{44}-\frac{R}{4}$, obtemos

$$
\left|h_{a b}-\frac{H_{\Sigma_{c}}}{3} g_{a b}\right|^{2}=\frac{(1+f)^{2}}{|\nabla f|^{2}}\left\{|\stackrel{\circ}{R} i c|^{2}-\frac{4}{3}[\stackrel{\circ}{R} i c(N, N)]^{2}\right\} \text {. }
$$

Observação 5.9. Nas condições da proposição acima $\lambda_{1}=\lambda_{2}=\lambda_{3}$ se, e somente se, $T_{4 a a}=0$. De fato, pelo quarto item da Proposição 5.8, por (5.17) temos que $h_{a b}=\frac{H_{\Sigma_{c}}}{3} g_{a b}$, i.e, todos os pontos de $\Sigma_{c}$ são umbílicos. De fato, pelo quarto item da proposição acima, temos que, se o tensor $T$ for nulo, os pontos de $\Sigma_{c}$ serão todos pontos umbílicos.

Em seqüência, nosso objetivo é mostrar que o tensor de Ricci é do tipo Codazzi, isto é, $\nabla_{i} R_{j k}-\nabla_{j} R_{i k}=0$. Isso nos garantirá que o tensor de Cotton na métrica CPE $C_{i j k}=\nabla_{i} R_{j k}-\nabla_{j} R_{i k}=0$. Sendo o tensor de Cotton identicamente nulo, teremos que o tensor curvatura será harmônico e isso implica que a métrica CPE é Einstein pelo Teorema 5.4 ([19]). Vejamos adiante os passos da demonstração. 
Teorema 5.10. Seja $\left(M^{4}, g, f\right)$ uma métrica CPE satisfazendo (1.26) tal que $\delta W^{+}=0$. Seja $c \neq-1$ um valor regular de $f$ e $\Sigma_{c}=\left\{x \in M^{4} ; f(x)=c\right\}$ os conjuntos de nivel de $f$. Tome $N=e_{4}=\frac{\nabla f}{|\nabla f|}$ e escolha um referencial ortonormal $\left\{e_{1}, e_{2}, e_{3}\right\}$ tangente a $\Sigma_{c}$, que diagonaliza o tensor de Ricci, isto é, $R_{i j}=\lambda_{i} \delta_{i j}$ para $1 \leq i, j \leq 4$. Suponha que $\lambda_{1}=\lambda_{2}=\lambda_{3}$, isto é, em $\left(M^{4}, g, f\right)$ o tensor de Ricci tem um autovalor de multiplicidade 1 e outro autovalor de multiplicidade 3. Então, a métrica g é Einstein, isto é, Ric $=\frac{R}{4} g$.

Demonstração. Como $\delta W^{+}=0$, pela Proposição 5.8, podemos escolher um referencial ortonormal $\left\{e_{1}, e_{2}, e_{3}, e_{4}\right\}$ com $N=e_{4}=\frac{\nabla f}{|\nabla f|}$ tal que $\left\{e_{1}, e_{2}, e_{3}\right\}$ é tangente a $\Sigma_{c}=$ $\left\{x \in M^{4} ; f(x)=c\right\}$, que diagonaliza o tensor de Ricci em algum valor regular de $f$, isto é, $R_{i j}=\lambda_{i} \delta_{i j}$ para $1 \leq i, j \leq 4$. Como vimos logo acima, pelo Lema 5.6, $\operatorname{Ric}\left(\nabla f, e_{a}\right)=g\left(\operatorname{Ric}(\nabla f), e_{a}\right)=\lambda g\left(\nabla f, e_{a}\right)$ e, claramente, $\nabla_{a} f=g\left(\nabla f, e_{a}\right)=0$, para $a, b, c=\{1,2,3\}$, então o tensor $T$ definido por (5.6) em $\Sigma_{c}$ é

$$
\begin{aligned}
T_{a b c} & =\frac{3}{2}\left(R_{a c} \nabla_{b} f-R_{b c} \nabla_{a} f\right)-\frac{1}{2}\left(R_{a s} \nabla^{s} f g_{b c}-R_{b s} \nabla^{s} f g_{a c}\right) \\
& -\frac{R}{2}\left(\nabla_{b} f g_{a c}-\nabla_{a} f g_{b c}\right) \\
& =\frac{3}{2}\left(\operatorname{Ric}\left(e_{a}, e_{c}\right) g\left(\nabla f, e_{b}\right)-\operatorname{Ric}\left(e_{b}, e_{c}\right) g\left(\nabla f, e_{a}\right)\right) \\
& -\frac{1}{2}\left(\lambda g\left(\nabla f, e_{a}\right) g_{b c}-\lambda g\left(\nabla f, e_{b}\right) g_{a c}\right) \\
& -\frac{R}{2}\left(g_{a c} g\left(\nabla f, e_{b}\right)-g_{b c} g\left(\nabla f, e_{a}\right)\right)=0 .
\end{aligned}
$$

Portanto, pelo Lema (5.2) e (5.18) temos que

$$
(f+1) C_{a b c}=W_{a b c s} \nabla^{s} f .
$$

em $\Sigma_{c}$. Já demonstramos que $g\left(e_{a}, \operatorname{Ric}\left(e_{4}\right)\right)=\lambda g\left(\frac{\nabla f}{|\nabla f|}, e_{a}\right)=0$, logo o tensor de Weyl (1.8) nos dá

$$
\begin{aligned}
R_{a b c 4} & =W_{a b c 4}+\frac{1}{2}\left(R_{a c} g_{b 4}+R_{b 4} g_{a c}-R_{a 4} g_{b c}-R_{b c} g_{a 4}\right) \\
& -\frac{R}{6}\left(g_{b 4} g_{a c}-g_{a 4} g_{b c}\right)
\end{aligned}
$$

ou seja, $W_{a b c s} \nabla^{s} f=R_{a b c s} \nabla^{s} f$. Isso implica que,

$$
(f+1) C_{a b c}=R_{a b c s} \nabla^{s} f
$$

nos conjuntos de nível $\Sigma_{c}$.

Temos por hipótese que $\lambda_{1}=\lambda_{2}=\lambda_{3}=\mu$ e $\lambda_{4}=\lambda$, são os respectivos autovalores 
associados a base $\left\{e_{1}, e_{2}, e_{3}, e_{4}=\frac{\nabla f}{|\nabla f|}\right\}$, como nas condições da Proposição 5.8. Então (5.16) nos dá

$$
\begin{aligned}
h_{a b} & =g\left(\nabla_{a} e_{b}, N\right)=-\frac{1}{|\nabla f|} g\left(e_{b}, \nabla_{a} \nabla f\right)=-\frac{\nabla_{a} \nabla_{b} f}{|\nabla f|} \\
& =\frac{1}{|\nabla f|}\left[\frac{R f}{12}-(1+f)\left(\lambda_{a}-\frac{R}{4}\right)\right] g_{a b} \\
& =\frac{1}{|\nabla f|}\left[\frac{R f}{12}-(1+f)\left(\mu-\frac{R}{4}\right)\right] g_{a b} .
\end{aligned}
$$

Logo, a curvatura média de $\Sigma_{c}$ é

$$
\begin{aligned}
H_{\Sigma_{c}}=\sum_{a=1}^{3} h_{a a} & =\frac{1}{|\nabla f|}\left[\frac{R f}{12}-(1+f)\left(\mu-\frac{R}{4}\right)\right] g_{a a} \\
& =\frac{3}{|\nabla f|}\left[\frac{R f}{12}-(1+f)\left(\mu-\frac{R}{4}\right)\right]
\end{aligned}
$$

Isso implica que

$$
\frac{H_{\Sigma_{c}}}{3}=\frac{1}{|\nabla f|}\left[\frac{R f}{12}-(1+f)\left(\mu-\frac{R}{4}\right)\right]
$$

isto é, por (5.23) e (5.22)

$$
h_{a b}=\frac{H_{\Sigma_{c}}}{3} g_{a b}
$$

Agora, nós usaremos a equação de Codazzi

$$
R_{4 c a b}=\nabla_{a} h_{b c}-\nabla_{b} h_{a c}
$$

para mostrar que $\lambda$ é constante em $\Sigma_{c}$. De fato, tomando o traço sobre $c$ e $b$ em (5.25), por $(5.24)$

$$
\begin{aligned}
0 & =R_{4 a}=g^{c b} R_{4 c a b}=\nabla_{a} g^{c b} h_{c b}-g^{c b} \nabla_{b} h_{a c}=\nabla_{a} H_{\Sigma_{c}}-\nabla_{b}\left(h_{a b}\right) \\
& =\nabla_{a} H_{\Sigma_{c}}-\frac{1}{3} \nabla_{a} H=\frac{2}{3} \nabla_{a} H_{\Sigma_{c}} .
\end{aligned}
$$

Isso implica que a curvatura média $H_{\Sigma_{c}}$ é constante no conjunto de nível $\Sigma_{c}$. Além disso, como o gradiente da função potential é autovetor de $\operatorname{Ric}$, isto é, $\stackrel{\circ}{\operatorname{Ric}}(N, N)=\left(\lambda-\frac{R}{4}\right)$, pela equação fundamental (1.26) e por (1.27) a curvatura média também pode ser escrita 
como

$$
\begin{aligned}
H_{\Sigma_{c}} & =\sum_{a=1}^{3} h_{a a}=\frac{1}{|\nabla f|}\left(\nabla^{2} f(N, N)-\Delta f\right) \\
& =\sum_{a=1}^{3} h_{a a}=\frac{1}{|\nabla f|}\left[(1+f)\left(\lambda-\frac{R}{4}\right)-\frac{R f}{12}+\frac{R f}{3}\right] \\
& =\frac{1}{|\nabla f|}\left[(1+f) \lambda-\frac{R}{4}\right]
\end{aligned}
$$

Isto é, $\lambda$ e $\mu$ (por (5.23) e (5.26)) são constante nos conjuntos de nível, já que $R,|\nabla f|^{2}$ e $H$ são constante nos conjuntos de nível da métrica CPE. Então, novamente por (5.25), (5.22) e pelo fato da diferencial covariante do tensor métrico ser igual a zero, temos $R_{a b c 4}=0$. Logo, por (5.20) temos que $W_{a b c 4}=R_{a b c 4}=0$. Isso implica por (5.21) que

$$
(f+1) C_{a b c}=W_{a b c s} \nabla^{s} f=R_{a b c s} \nabla^{s} f=0,
$$

e pela Proposição 4.2 obtemos $C_{a b c}=0 \mathrm{em} \Sigma_{c}$. Portanto, resta-nos saber o que acontece com o tensor de Cotton nas direções normais. Lembrando que precisamos demonstrar que $C_{i j k}=0 \mathrm{em} M^{4}$.

Primeiro, observe que $C_{a b 4}=0$. De fato, já que pela anti-simetria do tensor de Weyl

$$
W_{i j k s} \nabla^{k} f \nabla^{s} f=0
$$

e por $(5.11)$

$$
T_{i j k} \nabla^{k} f=\left(R_{i k} \nabla_{j} f-R_{j k} \nabla_{i} f\right) \nabla^{k} f
$$

Conseqüentemente, já que $\operatorname{Ric}(\nabla f)=\lambda \nabla f$, isto é, $R_{i j} \nabla^{j} f=\lambda \nabla_{i} f$, temos que

$$
T_{i j k} \nabla^{k} f=\lambda\left(\nabla_{i} f \nabla_{j} f-\nabla_{i} f \nabla_{j} f\right)=0
$$

Esse fato, juntamente com (5.27) e (5.7) garante que $C_{i j 4}=0$. Analogamente, temos que $T_{4 i 4}=0$. De fato, como $R_{i j} \nabla^{j} f=\lambda \nabla_{i} f$ e $\nabla_{i} f \nabla^{i} f=g^{i s} \nabla_{i} f \nabla_{s} f=|\nabla f|^{2}$

$$
\begin{aligned}
T_{i j k} \nabla^{i} f \nabla^{k} f & =\lambda\left(\nabla_{i} f \nabla_{j} f-\nabla_{j} f \nabla_{i} f\right) \nabla^{i} f \\
& =\lambda|\nabla f|^{2}\left(\nabla_{j} f-\nabla_{j} f\right)=0
\end{aligned}
$$

Portanto, pelas considerações feitas logo acima, pela equação (5.7), pela Proposição 4.2 
e pela anti-simetria do tensor de Weyl temos que

$$
C_{i j 4}=0 \quad \text { e } \quad C_{4 i 4}=-C_{i 44}=0
$$

no conjunto de nível $\Sigma_{c}$. Nos resta saber o que acontece com $C_{4 a b}$, já que pela antisimetria do tensor de Cotton $C_{44 a}=0$.

Agora, por (1.12) e (1.25) sabemos que

$$
4 \delta W_{j k l}^{+}=C_{k l j}+C_{\bar{k} \bar{l} j}
$$

Já que $\delta W_{j k l}^{+}=0$ por hipótese e $C_{a b c}=0$, obtemos $C_{\bar{a} \bar{b} c}=0$ por (5.28). Logo, por (1.19) temos que

$$
0=C_{\overline{1} \overline{2} c}=C_{34 c}, \quad 0=C_{\overline{1} \overline{3} c}=-C_{24 c} \quad \text { e } \quad 0=C_{\overline{2} \overline{3} c}=C_{14 c} .
$$

Finalmente, temos que $C_{i j k}=\nabla_{i} R_{j k}-\nabla_{j} R_{i k}=0, \forall p \in M$. Isso implica que tensor curvatura é harmônico, isto é $\nabla^{i} R_{i j k l}$. De fato, pela segunda identidade de Bianchi

$$
\nabla_{s} R_{i j k l}+\nabla_{i} R_{j s k l}+\nabla_{j} R_{s i k l}=0
$$

contraindo em $l$ e $s$, temos

$$
\begin{aligned}
& g^{l s} \nabla_{s} R_{i j k l}+g^{l s} \nabla_{i} R_{j s k l}+g^{l s} \nabla_{j} R_{s i k l} \\
= & \nabla^{l} R_{i j k l}+\nabla_{i} R_{j k}-\nabla_{j} R_{i k}=0 .
\end{aligned}
$$

Portanto, reorganizando os índices,

$$
\nabla^{i} R_{i j k l}=-\left(\nabla_{j} R_{k l}-\nabla_{k} R_{j l}\right)=-C_{j k l}=0
$$

veja também [19] para mais detalhes. Logo, pelo Teorema 5.4, $M^{4}$ é Einstein.

Certos resultados sobre métrica CPE usam algumas estimativas e igualdades integrais como hipótese. Podemos, por exemplo, ver alguns teoremas desse tipo em [35] e [8]. Em [35], Hwang prova alguns teoremas sob a hipótese adicional de que o tensor de Ricci satisfaz $R i c \geq \frac{R}{(1+f) n}$. E em [8], Benjamin usa algumas igualdades e desigualdades integrais na tentativa de se responder a conjectura. Motivados por esse tipo de resultado, vamos obter o Teorema 5.12 abaixo.

Corolário 5.11. Seja $\left(M^{4}, g, f\right)$ uma métrica CPE satisfazendo (1.26) tal que $\delta W^{+}=0$. 
Então,

$$
\frac{4}{3} \stackrel{\circ}{\operatorname{Ric}}(N, N)^{2} \leq|\stackrel{\circ}{\operatorname{Ric}}|^{2}
$$

Em particular, a métrica g é uma métrica de Einstein se, e somente se, ocorre a igualdade em (5.29).

Demonstração. Pelo quarto item da Proposição 5.8, vemos que

$$
0 \leq|\nabla f|^{2}\left|h_{a b}-\frac{H_{\Sigma_{c}}}{3} g_{a b}\right|^{2}=(1+f)^{2}\left(|\stackrel{\circ}{R} i c|^{2}-\frac{4}{3} \stackrel{\circ}{R} i c(N, N)^{2}\right) .
$$

Donde segue o resultado.

Se a igualdade ocorre em (5.29), temos que $h_{a b}=\frac{H_{\Sigma_{c}}}{3} g_{a b}$ (isto é, todos os pontos de $\Sigma_{c}$ são umbílicos). Agora demonstração do corolário segue os passos do teorema 5.10.

Antes de provar o nosso próximo resultado, vamos precisar de alguns fatos preliminares. Pela equação fundamental da métrica CPE (1.26)

$$
(1+f) \stackrel{\circ}{R}_{i j} \nabla^{j} f=\nabla_{i} \nabla_{j} f \nabla^{j} f+\frac{R f}{12} g_{i j} \nabla^{j} f=\frac{1}{2} \nabla_{i}\left(|\nabla f|^{2}+\frac{R f^{2}}{12}\right)
$$

Vamos denominar, assim como no capítulo anterior (veja Lema 4.7), $G=|\nabla f|^{2}+\frac{R f^{2}}{12}$. Portanto, por (5.30) temos

$$
2(1+f) \stackrel{\circ}{\operatorname{Ric}}(\nabla G, \nabla f)=|\nabla G|^{2} \quad \text { e } \quad 2(1+f) \stackrel{\circ}{\operatorname{Ric}}(\nabla f, \nabla f)=g(\nabla G, \nabla f) .
$$

O Lema 5.2 nos dá diretamente que (confira também Lema 3.1 em [19])

$$
\begin{aligned}
(1+f) C_{i j k} & =W_{i j k s} \nabla^{s} f-\frac{n-1}{n-2}\left(\nabla_{i} f \stackrel{\circ}{R}_{j k}-\nabla_{j} f \stackrel{\circ}{R}_{i k}\right) \\
& -\frac{1}{n-2}\left(\stackrel{\circ}{R}_{i s} \nabla^{s} f g_{j k}-\stackrel{\circ}{R}_{j s} \nabla^{s} f g_{i k}\right) .
\end{aligned}
$$

Substituindo (5.30) em (5.32) obtemos

$$
\begin{aligned}
2(1+f)^{2} C_{i j k} \nabla^{k} f & =2(1+f) W_{i j k s} \nabla^{k} f \nabla^{s} f-2(1+f) \frac{n-1}{n-2}\left(\nabla_{i} f \stackrel{\circ}{R}_{j k}-\nabla_{j} f \stackrel{\circ}{R}_{i k}\right) \nabla^{k} f \\
& -2(1+f) \frac{1}{n-2}\left(\stackrel{\circ}{R}_{i s} \nabla^{s} f g_{j k}-\stackrel{\circ}{R}_{j s} \nabla^{s} f g_{i k}\right) \nabla^{k} f \\
& =2(1+f)\left(\stackrel{\circ}{R}_{i s} \nabla^{s} f \nabla_{j} f-\stackrel{\circ}{R}_{i s} \nabla^{s} f \nabla_{j} f\right) \\
& =\nabla_{i} G \nabla_{j} f-\nabla_{j} G \nabla_{i} f .
\end{aligned}
$$


Se tomarmos o produto interno Hilbert-Schmidt na equação acima teremos que

$$
\begin{aligned}
4(1+f)^{4}\left|C_{i j k} \nabla^{k} f\right|^{2} & =\left|\nabla_{i} G \nabla_{j} f-\nabla_{j} G \nabla_{i} f\right|^{2} \\
& =g^{i k} g^{j l}\left(\nabla_{i} G \nabla_{j} f-\nabla_{j} G \nabla_{i} f\right)\left(\nabla_{k} G \nabla_{l} f-\nabla_{l} G \nabla_{k} f\right) \\
& =2|\nabla G|^{2}|\nabla f|^{2}-2 g(\nabla G, \nabla f)^{2} .
\end{aligned}
$$

Por outro lado, como o gradiente da função potencial é um autovetor do tensor de Ricci em $\left(M^{4}, g, f\right)$ quando $\delta W^{+}=0$ (Lema 5.6), seguindo os passos da demonstração do Teorema 5.10 temos

$$
C_{i j k} \nabla^{k} f=0
$$

Portanto, por (5.31) e (5.33) obtemos

$$
|\nabla G|^{2}|\nabla f|^{2}=g(\nabla G, \nabla f)^{2}=4(1+f)^{2}[\stackrel{\circ}{\operatorname{Ric}}(\nabla f, \nabla f)]^{2}
$$

Isto é,

$$
|\nabla G|^{2}=4(1+f)^{2}|\nabla f|^{2}[\stackrel{\circ}{\operatorname{Ric}}(N, N)]^{2}
$$

Agora estamos prontos para provarmos o próximo teorema.

Teorema 5.12. Seja $\left(M^{4}, g, f\right)$ uma métrica CPE satisfazendo (1.26) tal que $\delta W^{+}=0$. Então,

$$
\int_{M^{4}}|\nabla f|^{2} \stackrel{\circ}{\operatorname{Ric}}(\nabla f, \nabla f) \leq \frac{R^{2}}{36} \int_{M^{4}} G f^{2}
$$

onde $G=|\nabla f|^{2}+\frac{R f^{2}}{12}$. Em particular, a métrica $g$ é uma métrica de Einstein quando ocorre a igualdade.

Demonstração. Pelo Lema 4.7, por (5.34) e pelo quarto item da Proposição (5.8)

$$
\begin{aligned}
|\nabla f|^{4}\left|h_{a b}-\frac{H_{\Sigma_{c}}}{3} g_{a b}\right|^{2} & =(1+f)^{2}|\nabla f|^{2}|\stackrel{\circ}{\operatorname{R} i c \mid}|^{2}-\frac{4}{3}(1+f)^{2}|\nabla f|^{2}[\stackrel{\circ}{\operatorname{R} i c}(N, N)]^{2} \\
& =|\nabla f|^{2}\left(\frac{1}{2} \Delta G-\stackrel{\circ}{\operatorname{Ric}}(\nabla f, \nabla f)\right)-\frac{1}{3}|\nabla G|^{2} .
\end{aligned}
$$

Como $G=|\nabla f|^{2}+\frac{R f^{2}}{12}$ temos que

$$
|\nabla f|^{4}\left|h_{a b}-\frac{H_{\Sigma_{c}}}{3} g_{a b}\right|^{2}=\left(G-\frac{R f^{2}}{12}\right) \frac{1}{2} \Delta G-|\nabla f|^{2} \stackrel{\circ}{R} i c(\nabla f, \nabla f)-\frac{1}{3}|\nabla G|^{2} .
$$


Agora, vamos integrar sobre $M^{4}$ a equação acima. Pela fórmula de Stokes,

$$
\begin{aligned}
\int_{M^{4}}|\nabla f|^{4}\left|h_{a b}-\frac{H_{\Sigma_{c}}}{3} g_{a b}\right|^{2} & =\int_{M^{4}}\left(G-\frac{R f^{2}}{12}\right) \frac{1}{2} \Delta G-\int_{M^{4}}|\nabla f|^{2} \stackrel{\circ}{R} i c(\nabla f, \nabla f)-\int_{M^{4}} \frac{1}{3}|\nabla G|^{2} \\
& =-\frac{1}{2} \int_{M^{4}}|\nabla G|^{2}+\frac{R}{24} \int_{M^{4}} g\left(\nabla f^{2}, \nabla G\right) \\
& -\int_{M^{4}}|\nabla f|^{2} \stackrel{\circ}{\operatorname{Ric}}(\nabla f, \nabla f)-\int_{M^{4}} \frac{1}{3}|\nabla G|^{2} \\
& \leq-\frac{R}{24} \int_{M^{4}} G \Delta f^{2}-\int_{M^{4}}|\nabla f|^{2} \stackrel{\circ}{\operatorname{Ric}}(\nabla f, \nabla f) \\
& \leq-\frac{R}{12} \int_{M^{4}} G\left(|\nabla f|^{2}+f \Delta f\right)-\int_{M^{4}}|\nabla f|^{2} \stackrel{\circ}{\operatorname{Ric}}(\nabla f, \nabla f)
\end{aligned}
$$

Mas temos que $G \geq 0$ e $-\Delta f=\frac{R f}{3}, \operatorname{logo}$

$$
\begin{aligned}
0 \leq \int_{M^{4}}|\nabla f|^{4}\left|h_{a b}-\frac{H_{\Sigma_{c}}}{3} g_{a b}\right|^{2} & \leq-\frac{R}{12} \int_{M^{4}} G f \Delta f-\int_{M^{4}}|\nabla f|^{2} \stackrel{\circ}{\operatorname{Ric}}(\nabla f, \nabla f) \\
& =\frac{R}{12} \int_{M^{4}} G \frac{R f^{2}}{3}-\int_{M^{4}}|\nabla f|^{2} \stackrel{\circ}{\operatorname{Ric}}(\nabla f, \nabla f) \\
& =\frac{R^{2}}{36} \int_{M^{4}} G f^{2}-\int_{M^{4}}|\nabla f|^{2} \stackrel{\circ}{\operatorname{Ric}}(\nabla f, \nabla f) .
\end{aligned}
$$

Finalmente, se a igualdade ocorre em (5.35), temos que $h_{a b}=\frac{H}{3} g_{a b}$ (isso significa que todos os pontos de $\Sigma_{c}$ são umbílicos). Para concluir o teorema, basta repetirmos a demonstração do Teorema 5.10.

Os resultados desse capítulo podem ser encontrados em [5]. 


\section{Referências Bibliográficas}

[1] Anderson, M.: Scalar curvature, metric degenerations and the static vacuum Einstein equations on 3-manifolds. Geom. and Funct. Anal., 9 (1999), 855-967.

[2] Aubin, T.: Équation différentielles non-linéares et probléme de Yamabe concernant la courbure scalaire. J. Math. Pures Appl. 55 (1976) 269-296.

[3] Barbosa, E., Pina, P. and Tenenblat, K.: On Gradient Ricci Solitons conformal to a pseudo-Euclidean space, Israel Journal of Mathematics 200 (2014), 213-224.

[4] Barros, A. and Ribeiro Jr., E.: Critical point equation on four-dimensional compact manifolds. Math. Nachr. 287, N. 14-15, (2014) 1618-1623.

[5] Barros, A., Leandro, B. and Ribeiro Jr, E.: Critical metrics of the total scalar curvature functional on 4-manifolds, Math. Nachr. Volume 288, Issue 16, (2015) 1814-1821.

[6] Beem, J.K.: Some examples of incomplete space-times, Gen. Rel. Grav. 7, (1976), 501-509.

[7] Beem, J.K., Ehrlich, P.E. and Easley, K.L.: Global Lorentzian Geometry, Second edition, 1996 by Marcel Dekker, Inc.

[8] Benjamin Filho, F.: Remarks on critical point metrics of the total scalar curvature functional, Arch.Math, Springer Basel (2015).

[9] Besse, A.: Einstein manifolds, Springer-Verlag, Berlin Heidelberg (1987).

[10] Brozos-Vazquez, M., Garcia-Rio, E., Calvaruso, G. and Gavino-Fernandez, S.: Threedimensional Lorentzian homogeneous Ricci solitons, Isr. J. Math. 188, 385-403 (2012).

[11] Candela, A.M. and Sanchez, M.: Geodesics in semi-Riemannian Manifolds: Geometric Properties and Variational Tools, Recent developments in pseudo-Riemannian geometry, 359-418, ESI Lect. Math. Phys., Eur. Math. Soc., Zurich, 2008. 
[12] Calvino-Louzao, E., Seoane-Bascoy, J., Vázquez-Abal, M.E. and Vázquez-Lorenzo, R.: Three-dimensional homogeneous Lorentzian Yamabe solitons, Abh. Math. Semin. Univ. Hambg. (2012) 82: 193-203.

[13] Cao, H-D.: Recent progress on ricci solitons, arXiv:0908.2006v1 [math.DG] 14 Aug 2009.

[14] Cao, H-D. and Chen, Q.: On locally conformally flat gradient steady Ricci solitons Trans. Am. Math. Soc. 364-5, 2377-2391 (2012).

[15] Cao, H-D., Sun, X. and Zhang, Y.: On the structure of gradient Yamabe solitons, Math. Res. Lett. 19 (2012), no. 4, 767-774.

[16] Cao, H-D and Zhou, D.: On complete gradient shrinking Ricci solitons J. Differential Geom. Volume 85, Number 2 (2010), 175-186.

[17] Catino, G.: A note on four-dimensional (anti-)self-dual quasi-Einstein manifolds, Differential Geom. Appl. (2012), 660-664.

[18] Chang, J., Hwang, S. and Yun, G.: Rigidity of the critical point equation. Math. Nachr. 283 (2010) 846-853.

[19] Chang, J., Hwang, S. and Yun, G.: Total scalar curvature and harmonic curvature. Taiwanese Journal of Mathematics Vol. 18, N. 5, pp. 1439-1458, October (2014).

[20] Chang, J., Hwang, S. and Yun, G.: Critical point metrics of the total scalar curvature. Bull. Korean Math. Soc. 49 (2012) 655-667.

[21] Cheng, S.: Eigenvalues and nodal sets. Comment. Math. Helv. 51 (1976) 43-55.

[22] Cheng, X.: A generalization of almost-Schur lemma for closed Riemannian manifolds, Ann Glob Anal Geom (2013) 43: 153-160.

[23] Chen, X. and Wang, Y.: On four-dimensional anti-self-dual gradient Ricci solitons, J.Geom.Anal (2015) 25:1335-1343.

[24] Cheng, L. and Ma, L.: Properties of complete non-compact Yamabe solitons, Ann. Glob. Anal Geom. (2011), 40:379-387.

[25] Daskalopoulos, P. and Sesum, N.: The classification of locally conformally flat Yamabe solitons, Advances in Mathematics. 240 (2013), 346-369.

[26] Dillen, F. and Verstralen, L.: Handbook of Differential Geometry. Elsevier Science B. V. vol. 1 (2000). 
[27] Garcia-Rio, E., Brozos-Vazquez, M., Gavino-Fernandez, S. and Batat, W.: Ricci solitons on Lorentzian manifolds with large isometry groups, Bull. Lond. Math. Soc. 43,(2011) 1219-1227.

[28] Geroch, R.P.: What is a singularity in general relativity, Ann. Phys. (N.Y.) 48, (1968), 526-540.

[29] Hamilton, R.: The Ricci flow on surfaces, Contemporary Mathematics. 71, (1988) 237-261.

[30] Huang, G. and Li, H.: On a classification of the quasi Yamabe gradient solitons, Methods and Applications of Analysis, (2014) 21:379-390.

[31] Hsu, S-Y.: A note on compact gradient Yamabe solitons, J. Math. Anal. Appl. 388 (2012) 725-726.

[32] Hwang, S.: Characterizations of various classes of Einstein metrics, Ph.D. Thesis, SUNY at Stony Brook, 1996.

[33] Hwang, S.: The critical point equation on a three-dimensional compact manifold, Proc. of the Amer. Math. Soc. 131 (2003) 3221-3230.

[34] Hwang, S.: Three dimensional critical point of the total scalar curvature, Bull. Korean Math. Soc. 50 (2013), No. 3, pp. 867-871.

[35] Hwang, S.: Critical points of the total scalar curvature functional on the space of metrics of constant scalar curvature. Manuscripta Math. 103, (2000) 135 - 142.

[36] Hwang, S.: The critical point equation on a three-dimensional compact manifold. Proc. Amer. Math. Soc. 131 (2003) 3221-3230.

[37] Qing, j. and Yuan, W.: A note on static spaces and related problems, Journal of Geometry and Physics 74(2013) 18-27.

[38] Koiso, N.: A decomposition of the space of Riemannian metrics on a manifolds. Osaka J. of Math. 16, (1979) 423-429.

[39] Kuhnel, W.: Differential Geometry Curves- Surfaces- Manifolds, American Mathematical Society, vol. 16, Second edition.

[40] Kundt, W.: Note on the completeness of spacetimes, Zs. fur Phys. 172, (1963), 488-489. 
[41] Lafontaine, J.: Sur la géométrie d'une généralisation de l'équation différentielle d'Obata. J. Math. Pures Appliquées. 62 (1983) 63-72.

[42] Lee, J.M.: Introduction to smooth manifolds, University of Washington Department of Mathematics, Version 3.0 (2000).

[43] Lee, J.M. and Parker T. H.: The Yamabe problem, Bulletin of the American Mathematical Society Vol.17, number 1, July 1987.

[44] Leandro Neto, B.: A note on critical point metrics of the total scalar curvature functional. Journal of Math. Analysis and App. vol. 424, Issue 2, (2015) 1544-1548.

[45] Leandro, B. and Tenenblat, K.: On gradient Yamabe solitons conformal to a pseudoEuclidian space, (Trabalho Submetido).

[46] Leandro Neto, B.: A note on (anti-)self dual quasi Yamabe soliton. arXiv:1507.05974v2 [math.DG] 24 Jul 2015.

[47] Morrey, C. B.: Multiple Integrals in the Calculus of Variations, Séminaire de théorie Spectrale et Géométrie, Springer-Verlag Berlin, 1966.

[48] Obata, M.: Certain conditions for a Riemannian manifold to be isometric with a sphere. J. Math. Soc. Japan. 14, (1962) 333-340.

[49] Onda, K.: Lorentz Ricci solitons on 3-dimensional Lie groups, Geom. Dedic. 147, 313-322 (2010).

[50] O'neill, B.: Semi-Riemannian Geomtry with applications to relativity, American Press, New York, first edition, (1983).

[51] Olver, P. J.: Applications of Lie Groups to Differential Equations,, Second Edition, GTM 107 (2000).

[52] Qing, J. and Yuan, W.: A note on static spaces and related problems. J. of Geometry and Physics, 74, (2013) 18-27.

[53] Schoen, R.: Conformal deformation of a Riemannian metric to constant scalar curvature, J. Differential Geom. 20 (1984) 479-495.

[54] Scorpan, A.: The wild world of 4-manifolds. American Mathematical Society, Providence (1974).

[55] Trudinger, N.: Remarks concerning the conformal deformations of Riemannian structures on compact manifolds, Annali Scuola Norm. Sup. Pisa 22 (1968) 265274. 
[56] Viaclovsky, J.: Topics in Riemannian Geometry. Notes of Curse Math 865, Fall 2011, available at: http://www.math.wisc.edu/ jeffv/courses/865_Fall_2011.pdf

[57] Wang, L.F.: On noncompact quasi Yamabe gradient soliton, Differential Geometry and its Applications 31 (2013), 337-348.

[58] Wang, L.F.: The upper bound of the $L_{\mu}^{2}$ spectrum, Ann. Glob. Anal. Geom. 37 (4) (2010), 393-402. 